TRANSACTIONS OF THE

AMERICAN MATHEMATICAL SOCIETY

Volume 351, Number 9, Pages 3569-3607

S 0002-9947(99)02166-2

Article electronically published on April 26, 1999

\title{
A SYMPLECTIC JEU DE TAQUIN BIJECTION BETWEEN THE TABLEAUX OF KING AND OF DE CONCINI
}

\author{
JEFFREY T. SHEATS
}

\begin{abstract}
The definitions, methods, and results are entirely combinatorial. The symplectic jeu de taquin algorithm developed here is an extension of Schützenberger's original jeu de taquin and acts on a skew form of De Concini's symplectic standard tableaux. This algorithm is used to construct a weight preserving bijection between the two most widely known sets of symplectic tableaux. Anticipated applications to Knuth relations and to decomposing symplectic tensor products are indicated.
\end{abstract}

\section{INTRODUCTION}

The main result of this paper is a weight preserving bijection between two sets of symplectic tableaux. ${ }^{1}$ From either of these sets one can calculate the character of a given finite dimensional irreducible representation of the symplectic Lie algebra $\operatorname{sp}(2 n, \mathbb{C})$. To obtain the bijection, a jeu de taquin algorithm is developed for one kind of symplectic tableaux. The most famous application of Schützenberger's original jeu de taquin provided a bijective proof of the Littlewood-Richardson rule for the tensor product of two representations of $\operatorname{gl}(n, \mathbb{C})$. We expect that the symplectic jeu de taquin developed here will lead in the future to an analogous proof of Littelmann's rule for the tensor product of symplectic representations [Lit1].

Motivation begins with the problem of combinatorially describing characters of irreducible representations of reductive Lie algebras. In the case of $\operatorname{gl}(n, \mathbb{C})$, a solution is widely known. Given a partition $\lambda$ with no more than $n$ parts, the character $\operatorname{gl}_{n}(\lambda ; x)$ of the irreducible representation of $\operatorname{gl}(n, \mathbb{C})$ corresponding to $\lambda$ is the $\lambda$ th Schur function in the variables $x_{1}, x_{2}, \ldots, x_{n}$ (seen below as a quotient of $n \times n$ determinants). The set $\mathcal{T}(\lambda, n)$ of semistandard tableaux of shape $\lambda$ with entries from $\{\mathbf{1}, \mathbf{2}, \ldots, \mathbf{n}\}$ generate $\mathrm{gl}_{n}(\lambda ; x)$ :

$$
\operatorname{gl}_{n}(\lambda ; x)=: \frac{\left|x_{j}^{\lambda_{i}+n+1-i}\right|}{\left|x_{j}^{n+1-i}\right|}=\sum_{\mathcal{T} \in \mathcal{T}(\lambda, n)} x^{\mathrm{wt}(\mathcal{T})} .
$$

Received by the editors July 1, 1997.

1991 Mathematics Subject Classification. Primary 05E15, 22E46.

This research was supported in part by NSA Grants MDA 904-92-H-3061 and MDA 904-95H-1018.

${ }^{1}$ These results are contained in a doctoral thesis written under the supervision of Robert A. Proctor.

(C)1999 American Mathematical Society 
Analogously for $\operatorname{sp}(2 n, \mathbb{C})$, there are two historic tableau solutions:

$$
\begin{aligned}
\operatorname{sp}_{2 n}(\lambda ; x) & =: \frac{\left|x_{j}^{\lambda_{i}+n+1-i}-x_{j}^{-\left(\lambda_{i}+n+1-i\right)}\right|}{\left|x_{j}^{n+1-i}-x_{j}^{-(n+1-i)}\right|} \\
& =\sum_{\mathcal{T} \in \mathcal{D}(\lambda, n)} x^{\mathrm{wt}(\mathcal{T})}=\sum_{\mathcal{T} \in \mathcal{K}(\lambda, n)} x^{\mathrm{wt}(\mathcal{T})} .
\end{aligned}
$$

Combinatorialists are now more familiar with the sets $\mathcal{K}(\lambda, n)$. These were developed by Zhelobenko in 1961 in terms of Gelfand patterns [Zhl], and later converted by King to the tableaux used here [Kng]. Gelfand patterns have recently played a role in the far reaching program put forward by Berenstein and Zelevinsky concerning weight multiplicities and tensor product [BZ]. The sets we denote $\mathcal{D}(\lambda, n)$ were developed in 1979 by De Concini [DeC]. De Concini's tableaux are the symplectic case of a Lie algebra type-independent notion of tableaux developed by Laksmibai, Musili, and Seshadri in their series of " $G / P$ " papers [LMS]. As observed by Leclerc and Lebris, the $\mathcal{D}(\lambda, n)$ tableaux are essentially the same as those developed by Kashiwara and Nakashima to label elements of symplectic crystal bases $[\mathrm{KN}]$. The general linear case of the problem of relating Gelfand patterns to LMS-type tableaux essentially does not exist since the two forms are almost immediately equivalent. The symplectic case solved here is probably the easiest non-trivial case of this problem.

Our bijection problem is set in a fascinating class of difficult enumerative problems which has attracted the attention of many researchers over the last twenty years. This class is characterized by tableau enumeration formulas which, as products, are similar in form to the Weyl dimension formula (mysteriously so, in many cases). Although several pairs of sets of tableaux have been conjectured or proven to be equinumerous, few bijections have been found. For example, in 1986 Robbins made the famous conjecture that the set of totally symmetric self-complementary plane partitions is equinumerous with the set of monotone triangles [Rob]. In 1990 Andrews confirmed a product formula conjectured by Robbins to enumerate the former set [And]. In 1992 Zeilberger proved that the same product formula enumerated the latter [Zeil]. But the problem of finding a direct bijection is still open:

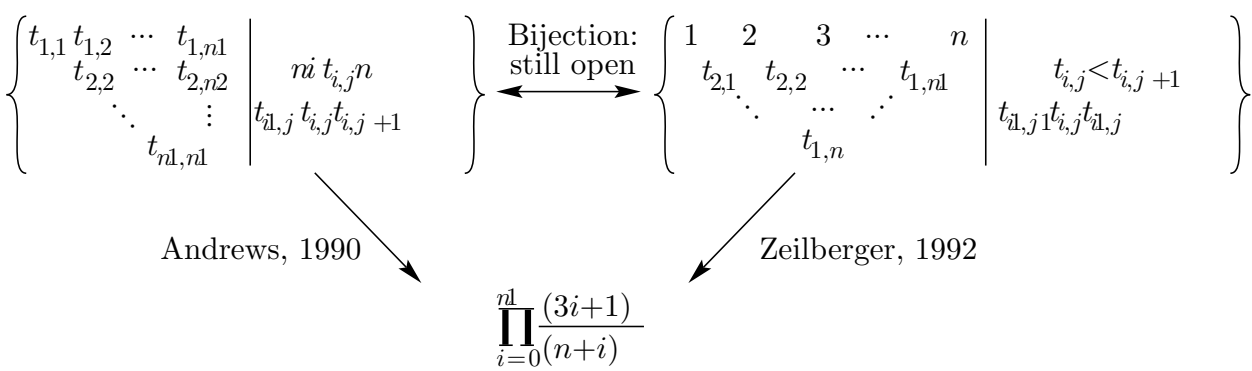


One bijection in this class of problems was due to Stanley [Sta]. He gave a bijection between the set of self-complementary plane partitions and a certain set of pairs of tableaux:

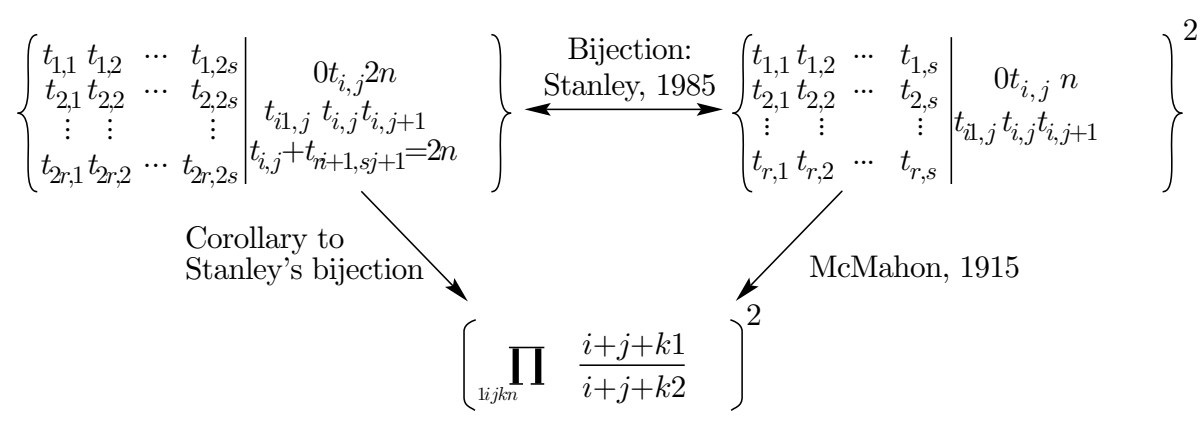

Stanley's bijection was based on a bijective proof of the Littlewood Richardson rule, and it could probably be reformulated with the jeu de taquin. In 1985 Proctor noted the coincidence of two tableau identities while considering $\mathcal{D}(\lambda, n)$ and $\mathcal{K}(\lambda, n)$ for certain special partitions $\lambda$ [Pro1]. A bijection between these two sets is a special case of our main result.

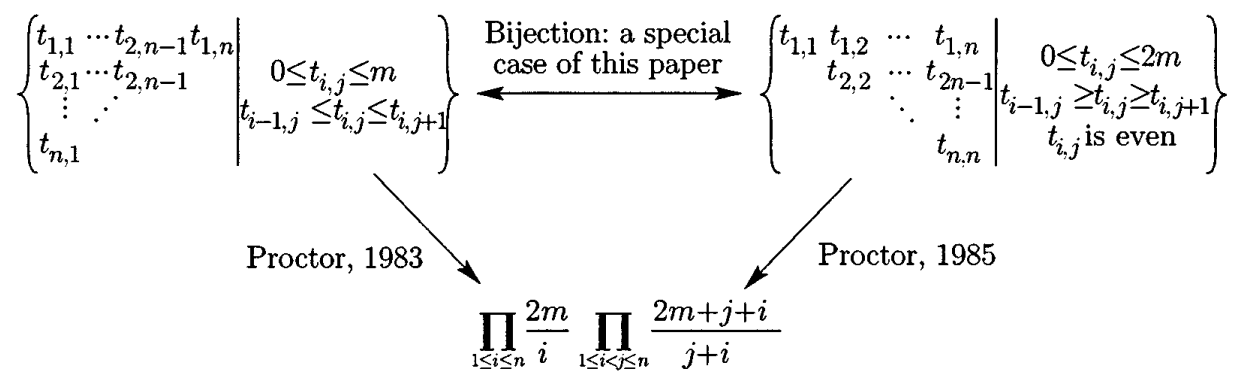

Aside from Stanley's result, the only other bijection in this class of problems known to us was recently found by Krattenthaler [Kra]. It uses the Garsia-Milne involution principle.

The problem of decomposing the tensor product of two irreducible representations into a sum of irreducible representations dates back to the late 19th century and the Clebsch-Gordon rule which solved the problem for gl $(2, \mathbb{C})$. In 1934 the Littlewood-Richardson rule provided a solution for $\operatorname{gl}(n, \mathbb{C})$. Not until 1988 did Littelmann, using some deep results from algebraic geometry, give generalized Littlewood-Richardson rules for the remaining classical Lie algebras [Lit1]. Recently, rules for all symmetrizable Kac-Moody algebras were found by Kashiwara [Kas] and Littelmann [Lit2]. In the mid-70's, with the development of the Schensted and the jeu de taquin algorithms, several beautiful bijective proofs of the original Littlewood-Richardson rule were made possible. We have empirical evidence that the symplectic jeu de taquin developed here will give rise to an analogous bijective proof of Littelmann's decomposition of symplectic tensor products. Earlier attempts at obtaining tensor product results with Berele's symplectic Schensted algorithm for King tableaux [Ber] succeeded only in some special cases [Sun], [Stro]. A possible explanation for this limited success is that Berele's algorithm does not 
seem to give a consistent set of generating "symplectic Knuth relations". The connection between Knuth relations and the original jeu de taquin is completely understood. Symplectic Knuth relations, based on constructions in $[\mathrm{KN}]$, were recently put forward by Lascoux, Leclerc, and Thibon [LLT]. Littelmann has also recently extended his program to give a version of such relations for all simple Lie algebras [Lit3]. The results contained in this paper were originally obtained in 1993 without knowledge of many of the advances in representation theory just mentioned. The development of the symplectic jeu de taquin algorithm was motivated entirely by the search for a weight preserving bijection between the two sets of symplectic tableaux. It will be interesting to explore the interplay between the concrete symplectic tableau jeu de taquin introduced in this paper and the more abstract constructions in [LLT] and [Lit3].

Donnelly has recently explicitly constructed all fundamental representations of $\operatorname{sp}(2 n, \mathbb{C})$. These appear to form the only known non-trivial infinite family of explicitly constructed representation of simple Lie algebras on weight bases aside from the Gelfand-Zetlin $\operatorname{gl}(n, \mathbb{C})$ construction of 1950. Reportedly the circle diagrams developed for weights of fundamental symplectic representations in Section 3 play a central role in Donnelly's construction.

In Section 2 we give basic definitions and a short preview of how the bijection works. In Sections 3, 4 and 5 the different types of tableaux are defined. In Section 6 the bijection problem is reduced to a sequence of easier problems. In Section 7 we define the symplectic jeu de taquin algorithm and state our main results. The proofs of our results are contained in Sections 8, 9, and 10. Section 11 presents conjectured applications of the symplectic jeu de taquin concerning: global well definedness, Knuth relations, the tensor product problem, and the generating functions for sets of skew De Concini tableaux. In the Appendix we relate the symplectic tableaux defined in [DeC] to those defined in [LMS, Lit1] and also to those defined in $[\mathrm{KN}]$. Skimmers should read the preview of the bijection in Section 2, Section 7 for the main results, and Section 11.

The author wishes to thank Phil Hanlon, John Stembridge, and the Michigan Department of Mathematics for their hospitality during the 1994-95 academic year. Special thanks go to Bob Proctor for his editorial assistance as well as his helpful comments on exposition.

\section{BASIC DEFINITIONS AND A PREVIEW}

Fix $n \geq 1$. A shape $\lambda$ is a weakly decreasing sequence $\lambda_{1} \geq \lambda_{2} \geq \cdots \geq \lambda_{n} \geq 0$ of non-negative integers. Let $l(\lambda)$ denote the number of non-zero entries in the sequence $\lambda$. We view the shape $\lambda$ as a collection of $l(\lambda)$ left justified rows of boxes with $\lambda_{i}$ boxes in the $i$ th row. A tableau of shape $\lambda$ is a filling of the boxes of the shape $\lambda$ with elements from a totally ordered set $\mathbf{S}$. Fix a tableau $\mathcal{T}_{\lambda}$ of shape $\lambda=\left(\lambda_{1}, \lambda_{2}, \ldots, \lambda_{n}\right)$. The entries of $\mathcal{T}_{\lambda}=\left\{\mathbf{t}_{i j}\right\}$ are indexed matrix style. We write $(i, j) \in \lambda$ if and only if $1 \leq i \leq l(\lambda)$ and $1 \leq j \leq \lambda_{i}$. A tableau with entries from $\mathbf{S}$ is said to be semistandard if (i) its entries in each column strictly increase from top to bottom, and (ii) its entries in each row weakly increase from left to right. Let $\mathbb{P}$ be the set of positive integers and let $[n]$ denote the subset $\{1,2, \ldots, n\}$. A signed tableaux of shape $\lambda$ is a filling of $\lambda$ with symbols from the set $[[\mathbb{P}]]=\{\overline{\mathbf{1}}, \mathbf{1}, \overline{\mathbf{2}}, \mathbf{2}, \overline{\mathbf{3}}, \mathbf{3}, \ldots\}$. The sets $\mathcal{D}(\lambda, n)$ and $\mathcal{K}(\lambda, n)$ are each collections of signed tableaux with entries from the subset $[[\mathbf{n}]]=\{\overline{\mathbf{1}}, \mathbf{1}, \overline{\mathbf{2}}, \mathbf{2}, \ldots, \overline{\mathbf{n}}, \mathbf{n}\}$. The 
weight $\operatorname{wt}(\mathcal{T})$ of a signed tableau $\mathcal{T}$ is the $n$-tuple $\left(j_{1}, j_{2}, \ldots, j_{n}\right)$, where $j_{i}$ is the number of i's minus the number of $\overline{\mathbf{i}}$ 's occurring in $\mathcal{T}$.

When $\lambda$ has more than one column the difference between the two sets $\mathcal{D}(\lambda, n)$ and $\mathcal{K}(\lambda, n)$ is very complicated. The difference stems primarily from the fact that different total orders on $[[\mathbf{n}]]$ are used. The total order used for De Concini's construction, denoted $<$, is the order inherited from the following total order on $[[\mathbb{P}]]$ :

$$
\cdots<\overline{\mathbf{3}}<\overline{\mathbf{2}}<\overline{\mathbf{1}}<\mathbf{1}<\mathbf{2}<\mathbf{3}<\cdots
$$

A signed tableau is semistandard if it is semistandard with respect to this total order. The total order $<_{1}$ on $[[\mathbb{P}]]$ associated to King's construction is given by

$$
\overline{\mathbf{1}}<_{1} \mathbf{1}<_{1}<\overline{\mathbf{2}}<_{1} \mathbf{2}<_{1} \overline{\mathbf{3}}<_{1} \mathbf{3}<_{1} \cdots \text {. }
$$

A signed tableau is called 1-semistandard if it is semistandard with respect to $<_{1}$. In order to obtain a bijection between $\mathcal{D}(\lambda, n)$ and $\mathcal{K}(\lambda, n)$ we create a sequence of intermediate sets of "hybrid" tableaux, each of which generate $\operatorname{sp}_{2 n}(\lambda ; x)$. The total order associated to each set is only slightly different than that of the next. For a fixed integer $d \geq 1$, the total order $<_{d}$ on $[[\mathbb{P}]]$ is given by

$$
\begin{aligned}
\overline{\mathbf{d}}<_{d} \overline{\mathbf{d}-\mathbf{1}} & <_{d} \cdots<_{d} \overline{\mathbf{1}}<_{d} \mathbf{1}<_{d} \mathbf{2} \\
& <_{d} \cdots<_{d} \mathbf{d}<_{d} \overline{\mathbf{d}+\mathbf{1}}<_{d} \mathbf{d}+\mathbf{1}<_{d} \overline{\mathbf{d}+\mathbf{2}}<_{d} \mathbf{d}+\mathbf{2}<_{d} \cdots .
\end{aligned}
$$

A signed tableau is $d$-semistandard if it is semistandard with respect to $<_{d}$. Note that a (no prefix) semistandard signed tableau whose entries are from $[[\mathbf{n}]]$ is also $d$-semistandard for all $d \geq n$.

Fix $n \geq 1$ and a fix a shape $\lambda$ with $l(\lambda) \leq n$. For an easier (general linear) preview of the first stage of the bijection $\mathcal{K}(\lambda, n) \rightarrow \mathcal{D}(\lambda, n)$, consider the subset of barred symbols $\overline{\mathbf{S}}=\{\overline{\mathbf{1}}, \overline{\mathbf{2}}, \ldots, \overline{\mathbf{n}}\}$ with the induced total orders $<_{n}$ and $<_{n-1}$ : $\overline{\mathbf{n}}<_{n} \overline{\mathbf{n}-\mathbf{1}}<_{n} \cdots<_{n} \overline{\mathbf{1}}$ and $\overline{\mathbf{n}-\mathbf{1}}<_{n-1} \overline{\mathbf{n}-\mathbf{2}} \cdots<_{n-1} \overline{\mathbf{1}}<_{n-1} \overline{\mathbf{n}}$. Let $\mathcal{T}^{n}(\lambda, n)$ and $\mathcal{T}^{n-1}(\lambda, n)$ be the sets of $n$-semistandard and $(n-1)$-semistandard tableaux of shape $\lambda$ with entries from $\overline{\mathbf{S}}$. The $\overline{\mathbf{n}}$ 's in a tableau of $\mathcal{T}^{n}(\lambda, n)$ form a one row subtableau in the northwest corner, while the $\overline{\mathbf{n}}$ 's in a tableau of $\mathcal{T}^{n-1}(\lambda, n)$ appear along its southeast perimeter. In this purely barred environment, Schützenberger's original jeu de taquin algorithm is sufficient to move these entries. The jeu de taquin is a procedure which slides an empty box from the inner subshape of a semistandard skew tableau to its southeast perimeter while preserving semistandardness. Define the map $\Gamma_{\lambda}^{n, n}: \mathcal{T}^{n}(\lambda, n) \rightarrow \mathcal{T}^{n-1}(\lambda, n)$ as follows. Given $\mathcal{T} \in \mathcal{T}^{n}(\lambda, n)$, temporarily remove all $\overline{\mathbf{n}}$ 's from $\mathcal{T}$ leaving a one row shape $(k)$ of empty boxes at the northwest corner of $\mathcal{T}$. Next, use the jeu de taquin $k$ times to slide out the empty boxes, keeping track of where they end up along the perimeter of the tableau. Define $\Gamma_{\lambda}^{n, n}(\mathcal{T})$ to be the tableau obtained by putting the $\overline{\mathbf{n}}$ 's back into these boxes. It is not hard to see that this process is bijective. Performing $n-2$ further such processes produces the set $\mathcal{T}^{1}(\lambda, n)$ of tableaux which are semistandard with respect to $<_{1}$ on $\overline{\mathbf{S}}: \overline{\mathbf{1}}<_{1} \overline{\mathbf{2}}<_{1} \cdots<_{1} \overline{\mathbf{n}}$. We will use the "symplectic jeu de taquin" algorithm to construct a bijection $\Psi_{\lambda}^{n, n}$ from $\mathcal{D}(\lambda, n)$ to the $(n-1)$ st intermediate set of symplectic tableaux. The restriction of $\Psi_{\lambda}^{n, n}$ to the subset $\mathcal{T}^{n}(\lambda, n)$ yields the map $\Gamma_{\lambda}^{n, n}$ defined above. Applying $\Psi_{\lambda}^{n, n-1}, \ldots, \Psi_{\lambda}^{n, 2}$ yields the bijection $\mathcal{D}(\lambda, n) \rightarrow$ $\mathcal{K}(\lambda, n)$.

The elements of $[[\mathbb{P}]]$ are thought of as abstract symbols. When referring to these elements, two levels of generality arise. Sometimes we may wish a variable 
to represent only barred (or only unbarred) symbols. For such a variable we use a barred (or unbarred) letter from the first part of the alphabet. E.g., $\overline{\mathbf{a}}, \overline{\mathbf{b}}, \ldots, \overline{\mathbf{d}}$ (or $\mathbf{a}, \mathbf{b}, \ldots, \mathbf{d})$. At other times a variable may denote an arbitrary element of $[[\mathbb{P}]]$. Then we use letters from the last part of the alphabet: $\boldsymbol{w}, \boldsymbol{x}, \ldots, \boldsymbol{z}$. To refer to the integer $d$ used in representing the symbol $\mathbf{d}$ or $\overline{\mathbf{d}}$, we use the map $\|\cdot\|:[[\mathbb{P}]] \rightarrow \mathbb{P}$ defined by $\|\overline{\mathbf{d}}\|=\|\mathbf{d}\|=d$. To represent subsets of $[[\mathbb{P}]]$ or signed tableaux we use capital script letters $\mathcal{P}, \mathcal{Q}, \ldots, \mathcal{T}$. The cardinality of any set $X$ is denoted by $|X|$. For two subsets $X$ and $Y$, we use $X-Y$ for $X \backslash Y$ when it is known that $Y \subseteq X$. Similarly, we use $X+Y$ for $X \cup Y$ when it is known that $X \cap Y$ is empty. For subsets $X=\left\{x_{1}<x_{2}<\cdots<x_{s}\right\}$ and $Y=\left\{y_{1}<y_{2}<\cdots<y_{t}\right\}$ of a totally ordered set, we say that $X \leq Y$ if: (i) $s \geq t$, and (ii) $x_{i} \leq y_{i}$ for $1 \leq i \leq t$. We extend this definition to semistandard one column tableaux by defining $\mathcal{P} \leq \mathcal{Q}$ if $\mathcal{P}$ can stand to the left of $\mathcal{Q}$ within a single semistandard tableau. For subsets of $\mathbb{P}$ we use ordinary capital letters: $A, B, C, \ldots$ A subshape $\mu$ of $\lambda$, written $\mu \subset \lambda$, is a shape such that $\mu_{i} \leq \lambda_{i}$ for all $i$. Asubtableau of $\mathcal{T}_{\lambda}=\left\{\mathbf{t}_{i j}\right\}$ is a tableau $\mathcal{S}_{\mu}=\left\{\mathbf{s}_{i j}\right\}$ of shape $\mu \subset \lambda$ such that $\mathbf{s}_{i j}=\mathbf{t}_{i j}$, for all $(i, j) \in \mu$. Given shapes $\mu \subset \lambda$, a skew tableau $\mathcal{T}_{\lambda \backslash \mu}$ of skew shape $\lambda \backslash \mu$ is a filling of the Ferrers diagram of $\lambda$ which leaves the boxes of $\mu$ empty.

The term "well defined" when used in connection with the ordinary jeu de taquin has a special meaning. In this paper, the "well definedness" of an algorithm will refer to different questions which were easy to answer for the ordinary jeu de taquin: Can the steps of the algorithm be unambiguously carried out? Is the output of the algorithm what it is claimed to be? In Section 11 we comment on the question of whether our symplectic jeu de taquin is globally well defined. That is, well defined in the special sense of the ordinary jeu de taquin.

\section{Admissible columns}

Understanding the relatively easy interrelationships amongst one column tableaux is essential for understanding the general bijection. In this section, for fixed $n \geq k \geq 1$, we define and relate the sets $\mathcal{D}\left(\left(1^{k}\right), n\right)$ and $\mathcal{K}\left(\left(1^{k}\right), n\right)$ of one column tableau. This involves the notion of "circle diagram".

A circle diagram is a method of viewing a subset of [[n]], or alternatively, a signed semistandard column. It is constructed on a $2 \times n$ grid. The squares in the top (bottom) row correspond to the barred (unbarred) elements. For example if $n=6$, then the subset $\{\overline{\mathbf{2}}, \overline{\mathbf{3}}, \overline{\mathbf{4}}, \overline{\mathbf{5}}, \mathbf{3}, \mathbf{5}, \mathbf{6}\}$ corresponds to the following circle diagram:

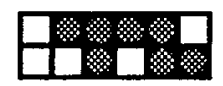

We call the pair of $b$ th squares from the left in the top and bottom rows the $b$ th slot. A slot is empty if it contains no circles, full if it contains two circles, and otherwise half-full. Note that $\operatorname{wt}(\mathcal{P})$ is determined by (and determines) the circles in the half-full slots. A $2 \times n$ circle diagram $\mathcal{P}$ is admissible if for each $m, 1 \leq m \leq n$, the first $m$ slots contain no more than $m$ circles. Equivalently, for all $m, 1 \leq m \leq n$, we have $|\{\mathbf{x} \in \mathcal{P}:\|\mathbf{x}\| \leq m\}| \leq m$. A one-column signed tableau $\mathcal{P}$ whose entries are from $[[\mathbf{n}]]$ is said to be admissible if it corresponds to a $2 \times n$ admissible circle diagram.

Fix $n \geq k \geq 1$. For any semistandard signed tableaux $\mathcal{P}$ of shape $\left(1^{k}\right)$ and entries from [[n] $]$, define $H_{\mathcal{P}}=\{a \in[n]$ : neither $\overline{\mathbf{a}}$ nor a occur in $\mathcal{P}\}$ (the empty 
slots) and $I_{\mathcal{P}}=\{b \in[n]$ : both $\overline{\mathbf{b}}$ and $\mathbf{b}$ occur in $\mathcal{P}\}$ (the full slots). Now we give the definition of De Concini's tableaux in the one column case. Let $\mathcal{D}\left(\left(1^{k}\right), n\right)$ be the set of all semistandard signed tableaux $\mathcal{P}$ of shape $\left(1^{k}\right)$ and entries from $[[\mathbf{n}]]$ such that $H_{\mathcal{P}} \leq I_{\mathcal{P}}$. Here are two semistandard columns and their corresponding circle diagrams with $n=6$.
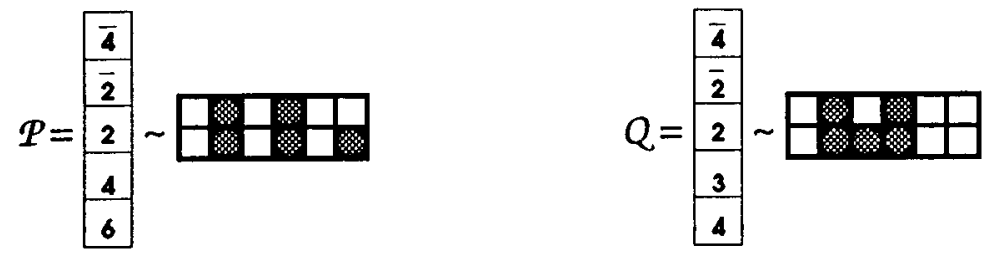

Here, $H_{\mathcal{P}}=\{1,3,5\}$ and $I_{\mathcal{P}}=\{2,4\}$ implies $\mathcal{P} \in \mathcal{D}\left(\left(1^{5}\right), 6\right)$; while $H_{\mathcal{Q}}=\{1,5,6\}$ and $I_{\mathcal{Q}}=\{2,4\}$ implies $\mathcal{Q} \notin \mathcal{D}\left(\left(1^{5}\right), 6\right)$. The circle diagram corresponding to $\mathcal{P}$ is admissible while the one corresponding to $\mathcal{Q}$ is not. This exemplifies:

Lemma 3.1. Let $\mathcal{P}$ be a semistandard column of length $k$ whose entries are from $[[\mathbf{n}]]$. Then $\mathcal{P}$ is an element of $\mathcal{D}\left(\left(1^{k}\right), n\right)$ if and only if $\mathcal{P}$ is admissible.

Proof. Fix $n \geq 1$ and let $\mathcal{P}$ be an element of $\mathcal{D}\left(\left(1^{k}\right), n\right)$. We see that $H_{\mathcal{P}} \leq I_{\mathcal{P}}$ gives a matching of each full slot with an empty slot to its left. Fix $m \leq n$ and consider the first $m$ slots of the circle diagram for $\mathcal{P}$. Each slot not involved in the matching contains at most one circle. Hence there can be at most $m$ circles in the first $m$ slots. Now assume $\mathcal{P}$ is admissible. The smallest element of $I_{\mathcal{P}}$ is the location of the first full slot. The definition of admissibility implies that there must be an empty slot to its left. Thus, the smallest element of $H_{\mathcal{P}}$ is smaller than the smallest element of $I_{\mathcal{P}}$. Continuing inductively we get $H_{\mathcal{P}} \leq I_{\mathcal{P}}$, since $\left|H_{\mathcal{P}}\right| \geq\left|I_{\mathcal{P}}\right|$.

Note that we have proved that a circle diagram is admissible if and only if there is a matching which sends each full slot to an empty slot to its left.

Now we give King's definition. Let $\mathcal{K}\left(\left(1^{k}\right), n\right)$ be the set of all 1 -semistandard tableaux $\mathcal{P}$ with entries from $[[\mathbf{n}]]$ of shape $\left(1^{k}\right)$ satisfying the following condition: If $p_{b}$ is the $b$ th entry from the top in the column $\mathcal{P}$, then $p_{b} \geq_{1} \overline{\mathbf{b}}$.

Lemma 3.2. Fix a 1-semistandard column $\mathcal{P}$ of length $k$ whose entries are from $[[\mathbf{n}]]$. Then $\mathcal{P}$ is an element of $\mathcal{K}\left(\left(1^{k}\right), n\right)$ if and only if $\mathcal{P}$ is admissible.

Proof. First, note that for all $\boldsymbol{x}, \boldsymbol{y} \in[[\mathbf{n}]], \boldsymbol{x} \leq_{1} \boldsymbol{y}$ implies $\|\boldsymbol{x}\| \leq\|\boldsymbol{y}\|$. Conversely, we see that if $b \in[n]$ and $b \leq\|\boldsymbol{x}\|$, then $\mathbf{b} \leq_{1} \boldsymbol{x}$. Assume $\mathcal{P} \in \mathcal{K}\left(\left(1^{k}\right), n\right)$ and fix an $m, 1 \leq m \leq n$. If the set $\mathcal{P}_{m}:=\{\boldsymbol{x} \in \mathcal{P}:\|\boldsymbol{x}\| \leq m\}$ is empty, there is nothing to show. Otherwise, let $p_{b}$ (the $b$ th element from the top in $\mathcal{P}$ ) be the largest element of $\mathcal{P}_{m}$ (with respect to $\leq_{1}$ ). Then, $|\{\boldsymbol{x} \in \mathcal{P}:\|\boldsymbol{x}\| \leq m\}|=b$. Also, $p_{b} \geq_{1} \overline{\mathbf{b}}$ implies $b \leq\left\|p_{b}\right\|$. And $p_{b} \in \mathcal{P}_{m}$ implies $\left\|p_{b}\right\| \leq m$. So, $|\{\boldsymbol{x} \in \mathcal{P}:\|\boldsymbol{x}\| \leq m\}| \leq m$. Next assume $\mathcal{P}$ is admissible and fix a $b, 1 \leq b \leq k$. Let $m=\left\|p_{b}\right\|$, then $b \leq \mid\{x \in \mathcal{P}$ : $\|x\| \leq m\} \mid \leq m=\left\|p_{b}\right\|$. This implies $\overline{\mathbf{b}} \leq_{1} p_{b}$.

For fixed $n$ and $1 \leq k \leq n$, the tableaux of the sets $\mathcal{D}\left(\left(1^{k}\right), n\right)$ and $\mathcal{K}\left(\left(1^{k}\right), n\right)$ correspond to the same set of circle diagrams: the admissible ones with $k$ circles. Thus the bijection for the one column case $\mathcal{D}\left(\left(1^{k}\right), n\right) \rightarrow \mathcal{K}\left(\left(1^{k}\right), n\right)$ is trivial, consisting of merely re-ordering the entries. 


\section{The TABLEAUX}

Fix a positive integer $n$ and let $\lambda$ be a shape with $l(\lambda) \leq n$. Most of this section is devoted to defining the set $\mathcal{D}(\lambda, n)$. The set $\mathcal{K}(\lambda, n)$ has a simple definition: $\mathcal{K}(\lambda, n)$ is the set of all 1-semistandard tableaux of shape $\lambda$ with admissible columns and whose entries are from $[[\mathbf{n}]]$.

Let $\mathcal{T}$ be a semistandard tableau of shape $\lambda$ with entries from $[[\mathbf{n}]]$ and admissible columns $\mathcal{T}_{1}, \mathcal{T}_{2}, \ldots, \mathcal{T}_{c}$. Membership in $\mathcal{D}(\lambda, n)$ requires more than just the semistandardness of $\mathcal{T}$. It involves "splitting" each column $\mathcal{T}_{i}$ into two columns, a left "half" $l \mathcal{T}_{i}$ and a right "half" $r \mathcal{T}_{i}$. For $\mathcal{T}$ to be an element of $\mathcal{D}(\lambda, n)$ the column $\mathcal{T}_{i-1}$ may sit to the left of column $\mathcal{T}_{i}$ iff $r \mathcal{T}_{i-1} \leq l \mathcal{T}_{i}$. To obtain these two halves we must first break an admissible column into two parts and then generate two new parts.

Let $(X, Y)$ be any pair of finite subsets of $\mathbb{P}$. Define $F(X, Y)$ to be the semistandard column with entries $\{\overline{\mathbf{a}}: a \in X\} \cup\{\mathbf{d}: d \in Y\}$. If $\mathcal{P}=F(X, Y)$, the set $X$ lists the slots containing circles in the top row of the circle diagram for $\mathcal{P}$, while the set $Y$ lists the slots of the bottom row circles. For any column $\mathcal{P} \in \mathcal{D}\left(\left(1^{k}\right), n\right)$ denote by $A_{\mathcal{P}}$ and $D_{\mathcal{P}}$ the subsets of $[n]$ such that $\mathcal{P}=F\left(A_{\mathcal{P}}, D_{\mathcal{P}}\right)$. As in the definition for $\mathcal{D}\left(\left(1^{k}\right), n\right)$, set $I_{\mathcal{P}}=A_{\mathcal{P}} \cap D_{\mathcal{P}}$ and $H_{\mathcal{P}}=[n]-\left(A_{\mathcal{P}} \cup D_{\mathcal{P}}\right)$. Define $J_{\mathcal{P}}=\max \left[X \subseteq H_{\mathcal{P}}:|X|=\left|I_{\mathcal{P}}\right|\right.$ and $\left.X<I_{\mathcal{P}}\right]$. The set $J_{\mathcal{P}}$ is well defined: Since $H_{\mathcal{P}} \leq I_{\mathcal{P}}$, the set $\left\{X \subseteq H_{\mathcal{P}}:|X|=\left|I_{\mathcal{P}}\right|\right.$ and $\left.X<I_{\mathcal{P}}\right\}$ is non-empty. This latter set is also easily seen to be a finite partially ordered set closed under the join operation. So it has a maximum element. The two new parts we need are $B_{\mathcal{P}}=\left(A_{\mathcal{P}}-I_{\mathcal{P}}\right)+J_{\mathcal{P}}$ and $C_{\mathcal{P}}=\left(D_{\mathcal{P}}-I_{\mathcal{P}}\right)+J_{\mathcal{P}}$. For an admissible column $\mathcal{P}$ we refer to $A_{\mathcal{P}}, B_{\mathcal{P}}, C_{\mathcal{P}}, D_{\mathcal{P}}$, as the associated subsets. Define $l \mathcal{P}=F\left(A_{\mathcal{P}}, C_{\mathcal{P}}\right)$ and $r \mathcal{P}=F\left(B_{\mathcal{P}}, D_{\mathcal{P}}\right)$.

By their definition, one sees that $\left|A_{\mathcal{P}}\right|=\left|B_{\mathcal{P}}\right|$ with $A_{\mathcal{P}} \geq B_{\mathcal{P}}$ and $\left|C_{\mathcal{P}}\right|=\left|D_{\mathcal{P}}\right|$ with $C_{\mathcal{P}} \leq D_{\mathcal{P}}$. (Keep in mind that $A_{\mathcal{P}}, B_{\mathcal{P}}, C_{\mathcal{P}}, D_{\mathcal{P}}$ are subsets of $[n]$.) This implies that $l \mathcal{P} \leq \mathcal{P} \leq r \mathcal{P}$ when signed columns are formed. It is helpful to think of the "splitting" process schematically as $\underset{D}{A} \rightarrow \underset{C}{A} \underset{D}{B}$, where the entries corresponding to $A$ and $B$ are barred. As an example, let $n=6$ and let $\mathcal{P}$ be as shown below. Then $A_{\mathcal{P}}=\{5,6\}$ and $D_{\mathcal{P}}=\{2,4,5\}$ imply that $I_{\mathcal{P}}=\{5\}$ and $H_{\mathcal{P}}=\{1,3\}$. So we have $J_{\mathcal{P}}=\{3\}, B_{\mathcal{P}}=\{3,6\}$ and $C_{\mathcal{P}}=\{2,3,4\}$.

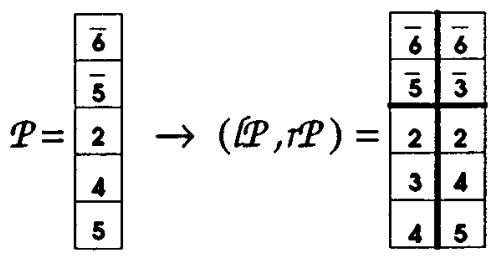

The pairs $(l \mathcal{P}, r \mathcal{P})$ correspond to admissible pairs of extreme weights developed in [LMS], as is discussed in the Appendix.

We are now able to define admissible tableaux and the set $\mathcal{D}(\lambda, n)$ : A signed tableau $\mathcal{T}$ with admissible columns $\mathcal{T}_{1}, \mathcal{T}_{2}, \ldots, \mathcal{T}_{c}$ is an admissible tableau if and only if the split column form of $\mathcal{T}$ with columns $l \mathcal{T}_{1}, r \mathcal{T}_{1}, l \mathcal{T}_{2}, r \mathcal{T}_{2}, \ldots, l \mathcal{T}_{c}, r \mathcal{T}_{c}$ is semistandard. Define $\mathcal{D}(\lambda, n)$ to be the set of all admissible tableau of shape $\lambda$ whose entries are from $[[\mathbf{n}]]$.

The problem with which this paper is concerned is: 
4.1. The bijection problem. For each positive integer $n$ and shape $\lambda$ with $l(\lambda) \leq$ $n$, give a weight preserving bijection between the sets $\mathcal{D}(\lambda, n)$ and $\mathcal{K}(\lambda, n)$.

The proof that our procedure is in fact a weight preserving bijection is self contained and independent of (1.1) the fact that the two sets have the same weight generating function. (One would need to confirm either only injectivity or surjectivity alone for our bijection if one were willing to rely upon this result.)

Fix shapes $\lambda$ and $\mu$ such that $\mu \subseteq \lambda$. Admissible skew tableaux and the set $\mathcal{D}(\lambda \backslash \mu, n)$ are defined as above: The empty boxes are ignored in determining admissible columns. Simply repeat the empty boxes when constructing the splitcolumn form of a given column. A skew tableau is admissible if its split-column form is semistandard. Here is an element of $\mathcal{D}\left(\left(3^{2}, 2,1\right) \backslash(2,1), 2\right)$ and its split-column form:
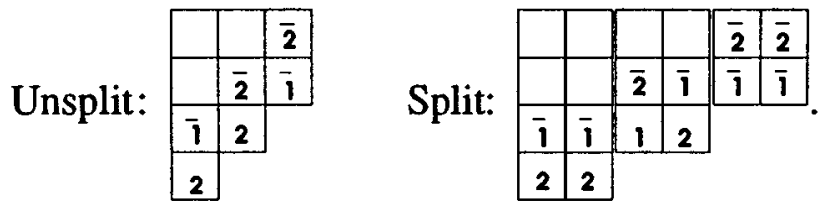

We use the following notation for the split-column form of an admissible (skew) tableau $\mathcal{T}$ with columns $\mathcal{T}_{1}, \mathcal{T}_{2}, \ldots, \mathcal{T}_{c}$ : The $p$ th pair of entries in the $q$ th splitcolumn $\left(l \mathcal{T}_{q}, r \mathcal{T}_{q}\right)$ is referred to as $(l, r)_{p, q}$. Individual entries are denoted $l_{p, q}$ and $r_{p, q}$. The corresponding locations are denoted $(p, q)_{l}$ and $(p, q)_{r}$.

\section{CoAdmissible COlumns}

Fix $n \geq k \geq 1$ and let $\mathcal{P} \in \mathcal{D}\left(\left(1^{k}\right), n\right)$ be an admissible column. We discuss the associated subsets $B_{\mathcal{P}}$ and $C_{\mathcal{P}}$ of an admissible column $\mathcal{P}=F\left(A_{\mathcal{P}}, D_{\mathcal{P}}\right)$. Recall the definition of the associated subsets $A_{\mathcal{P}}, B_{\mathcal{P}}, C_{\mathcal{P}}, D_{\mathcal{P}}$. The sets $B_{\mathcal{P}}$ and $C_{\mathcal{P}}$ are defined in terms of $A_{\mathcal{P}}$ and $D_{\mathcal{P}}$. The set $I_{\mathcal{P}}=A_{\mathcal{P}} \cap D_{\mathcal{P}}$ lists the full slots of the circle diagram for $\mathcal{P}$. Loosely speaking, the set $J_{\mathcal{P}}$ is the rightmost set of empty slots to the left of the full slots. The circle diagram for $F\left(B_{\mathcal{P}}, C_{\mathcal{P}}\right)$ is produced from the circle diagram for $F\left(A_{\mathcal{P}}, D_{\mathcal{P}}\right)$ by "jumping" the pairs of circles from the slots of $I_{\mathcal{P}}$ leftward to the slots of $J_{\mathcal{P}}$, leaving the half-full slots undisturbed. Conversely, $A_{\mathcal{P}}$ and $D_{\mathcal{P}}$ are determined by $B_{\mathcal{P}}$ and $C_{\mathcal{P}}: J_{\mathcal{P}}=B_{\mathcal{P}} \cap C_{\mathcal{P}}, I_{\mathcal{P}}=$ $\min \left[X \subseteq[n]-\left(B_{\mathcal{P}} \cup C_{\mathcal{P}}\right):|X|=\left|J_{\mathcal{P}}\right|\right.$ and $\left.X>J_{\mathcal{P}}\right], A_{\mathcal{P}}=\left(B_{\mathcal{P}}-J_{\mathcal{P}}\right)+I_{\mathcal{P}}$, and $D_{\mathcal{P}}=\left(C_{\mathcal{P}}-J_{\mathcal{P}}\right)+I_{\mathcal{P}}$. Now $J_{\mathcal{P}}$ lists the full slots of $F\left(B_{\mathcal{P}}, C_{\mathcal{P}}\right)$, while $I_{\mathcal{P}}$ lists the leftmost empty slots to the right of the full slots. The circle diagram for $F\left(A_{\mathcal{P}}, D_{\mathcal{P}}\right)$ is produced from that for $F\left(B_{\mathcal{P}}, C_{\mathcal{P}}\right)$ by "jumping" the pairs of circles from the slots of $J_{\mathcal{P}}$ rightward back to the slots of $I_{\mathcal{P}}$.

A $2 \times n$ circle diagram $Q$ is coadmissible if $|\{\boldsymbol{x} \in \mathcal{Q}:\|\boldsymbol{x}\| \geq n-m+1\}| \leq m$ for $1 \leq m \leq n$. That is, if for all $m$ there are no more than $m$ circles in the last $m$ slots. Let $\tau^{\prime}$ be the reflection acting on circle diagrams which reverses the order of the slots. Then a circle diagram $Q$ is coadmissible if and only if its reflected image $\tau^{\prime} \mathcal{Q}$ is admissible. To each statement concerning admissible circle diagrams there corresponds a reflected statement applicable to coadmissible circle diagrams. For example, a circle diagram is coadmissible if and only if there exists a matching which sends each full slot to an empty slot to its right. 
For fixed $k \geq 1$, define $\mathcal{D}\left(1^{k}\right)$ to be the set of all admissible columns of length $k$ : $\mathcal{D}\left(1^{k}\right):=\bigcup_{n} \mathcal{D}\left(\left(1^{k}\right), n\right)$. For a fixed shape $\lambda$ or fixed skew shape $\lambda \backslash \mu$ define $\mathcal{D}(\lambda)$ or $\mathcal{D}(\lambda \backslash \mu)$ analogously.

Lemma 5.1. Let $W$ and $Z$ be finite subsets of $\mathbb{P}$ and set $k=|W|+|Z|$. Then there exists a unique admissible column $\mathcal{P}$ in $\mathcal{D}\left(1^{k}\right)$ such that $B_{\mathcal{P}}=W$ and $C_{\mathcal{P}}=Z$.

Proof. If $\mathcal{Q}$ is any $2 \times n$ circle diagram there is always some $k \geq 0$ such that adjoining $k$ empty slots to the right produces a coadmissible $2 \times(n+k)$ circle diagram. Thus for any pair of finite subsets $W, Z \subset \mathbb{P}$ there exists an $N$ such that the $2 \times N$ circle diagram with top row circles listed by $W$ and bottom row circles listed by $Z$ is coadmissible. In other words there is an admissible column $\mathcal{P}$ in $\mathcal{D}\left(\left(1^{k}\right), N\right)$ such that $B_{\mathcal{P}}=W$ and $C_{\mathcal{P}}=Z$. It is clear that $\mathcal{P}$ is unique (even though $N$ is not).

Let $G$ denote the map $(W, Z) \mapsto \mathcal{P}$ suggested by Lemma 5.1. Suppose we are given an admissible column $\mathcal{P}$ at the outset and we produce $A_{\mathcal{P}}, B_{\mathcal{P}}, C_{\mathcal{P}}, D_{\mathcal{P}}$. Then $\mathcal{P}=F\left(A_{\mathcal{P}}, D_{\mathcal{P}}\right)$ and $\mathcal{P}=G\left(B_{\mathcal{P}}, C_{\mathcal{P}}\right)$. While $\mathcal{P}$ ranges over $\mathcal{D}\left(1^{k}\right)$, the pair $\left(B_{\mathcal{P}}, C_{\mathcal{P}}\right)$ ranges over all pairs of finite subsets of $\mathbb{P}$ whose sizes sum to $k$, and $\left(A_{\mathcal{P}}, D_{\mathcal{P}}\right)$ ranges over all such pairs which have the additional property that $F\left(A_{\mathcal{P}}, D_{\mathcal{P}}\right)$ is admissible. Note that the difference in circle diagrams corresponding to $\left(A_{\mathcal{P}}, D_{\mathcal{P}}\right)$ and $\left(B_{\mathcal{P}}, C_{\mathcal{P}}\right)$ is the location of the full slots, which do not affect weight. It follows that the induced map $F\left(A_{\mathcal{P}}, D_{\mathcal{P}}\right) \mapsto F\left(B_{\mathcal{P}}, C_{\mathcal{P}}\right)$ is a weight preserving bijection from $\mathcal{D}\left(1^{k}\right)$ to the set of all semistandard signed tableaux of shape $\left(1^{k}\right)$.

\section{Reducing the PROBLem}

We define the sets of intermediate tableaux mentioned in Section 2. Fix $d \geq 1$. A tableau $\mathcal{T}$ is in $\mathcal{M}^{d}(\lambda, n)$ if and only if (i) it is $d$-semistandard, (ii) has admissible columns, and (iii) the subtableau of $\mathcal{T}$, say of shape $\mu$, consisting of those entries which are less than or equal to $\mathbf{d}$ with respect to $\leq_{d}$ is an element of $\mathcal{D}(\mu, d)$.

Notice that when $d \geq n$ condition (iii) in the definition together with $l \mathcal{T}_{i} \leq \mathcal{T}_{i} \leq$ $r \mathcal{T}_{i}$ imply that (i) and (ii) are satisfied. Thus, $\mathcal{D}(\lambda, n)=\mathcal{M}^{n}(\lambda, n)$. When $d=1$, conditions (i) and (ii) imply (iii) is satisfied, so $\mathcal{K}(\lambda, n)=\mathcal{M}^{1}(\lambda, n)$. We call the subtableau of (iii) the De Concini-part of $\mathcal{T}$, and the remaining skew tableau the King-part of $\mathcal{T}$. Below is an element of $\mathcal{M}^{5}\left(\left(4^{3}, 2\right), 7\right)$ with its De Concini and King parts displayed:

\begin{tabular}{|c|c|c|c|}
\hline 5 & $\overline{2}$ & $\overline{6}$ & 6 \\
\hline$\overline{4}$ & $\overline{1}$ & 6 & $\overline{7}$ \\
\hline 4 & 6 & 7 & 7 \\
\hline$\overline{6}$ & $\overline{7}$ & \multicolumn{2}{|c}{} \\
\cline { 1 - 2 } & &
\end{tabular}

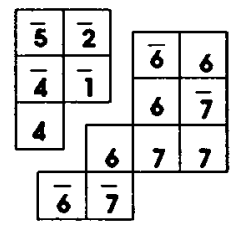

These intermediate "hybrid" tableaux inherit from their King-part the nice property of having interchangeable regions.

Lemma 6.1. Fix integers $a, b$, and $c$ in $[n]$ such that $a \leq c$ and $b \leq c$, and fix a tableau $\mathcal{T}_{\lambda}$ in $\mathcal{M}^{a}(\lambda, n)$. Denote by $\mathcal{T}_{\mu}$ the subtableau of shape $\mu$ formed by the entries of $\mathcal{T}_{\lambda}$ which are $\leq_{a}$ c. Choose any element $\mathcal{S}_{\mu}$ of $\mathcal{M}^{b}(\mu, c)$. Form the tableau $\mathcal{S}_{\lambda}$ by replacing $\mathcal{T}_{\mu}$ with $\mathcal{S}_{\mu}$ within the tableau $\mathcal{T}_{\lambda}$. Then $\mathcal{S}_{\lambda}$ is an element of $\mathcal{M}^{b}(\lambda, n)$. 
Proof. It is clear that $\mathcal{S}_{\lambda}$ satisfies conditions (i) and (iii) of the definition. To show (ii) is satisfied it is sufficient to consider the case when $\lambda$ is a single column. Fix $1 \leq k \leq n$, let $\lambda=\left(1^{k}\right)$ and let $\mathcal{P} \in \mathcal{M}^{a}\left(\left(1^{k}\right), n\right)$. In terms of circle diagrams, $\mathcal{P}$ has $n$ slots. Consider the first $c$ slots of $\mathcal{P}$. Suppose $j$ circles occur here (i.e., $\left.\mu=\left(1^{j}\right)\right)$. The lemma asserts that when these $c$ slots are replaced with any other admissible circle diagram with $c$ slots and $j$ circles, the resulting circle diagram is admissible. By the definition of admissibility, this is clearly true.

Now the bijection problem reduces to the problem of finding a sequence of $n-1$ weight preserving bijections $\mathcal{M}^{d}(\lambda, n) \rightarrow \mathcal{M}^{d-1}(\lambda, n), n \geq d>1$. Using the lemma above we reduce Problem 4.1 to the following.

6.2. The reduced bijection problem. For each positive integer $n$ and shape $\lambda$ with $l(\lambda) \leq n$, give a weight preserving bijection between the sets $\mathcal{M}^{n}(\lambda, n)$ and $\mathcal{M}^{n-1}(\lambda, n)$.

Proof. Suppose that $\Phi_{\lambda}^{n}: \mathcal{M}^{n}(\lambda, n) \rightarrow \mathcal{M}^{n-1}(\lambda, n)$ is a weight preserving bijection for each positive integer $n$ and shape $\lambda$ with no more than $n$ rows. For each $d, n \geq d \geq 1$, define the map $\Psi_{\lambda}^{n, d}: \mathcal{M}^{d}(\lambda, n) \rightarrow \mathcal{M}^{d-1}(\lambda, n)$ as follows. Let $\mathcal{T}_{\lambda} \in \mathcal{M}^{d}(\lambda, n)$ and let $\mathcal{T}_{\mu}$ be the De Concini part of $\mathcal{T}_{\lambda}$ of shape $\mu$. Then $\mathcal{T}_{\mu}$ is an element of $\mathcal{M}^{d}(\mu, d)$. Define $\Psi_{\lambda}^{n, d}\left(\mathcal{T}_{\lambda}\right)$ to be the tableau formed by replacing $\mathcal{T}_{\mu}$ with $\Phi_{\mu}^{d}\left(\mathcal{T}_{\mu}\right)$. That the map $\Psi_{\lambda}^{n, d}$ is well defined is an immediate consequence of Lemma 6.1. It is a trivial exercise to verify that $\Psi_{\lambda}^{n, d}$ is a weight preserving bijection. The bijection which solves Problem 4.1 is $\Psi_{\lambda}^{n, 2} \circ \Psi_{\lambda}^{n, 3} \circ \cdots \circ \Psi_{\lambda}^{n, n}$.

The $a=1, b=c=d$ case of Lemma 6.1 along with (1.1) can be used to prove the next proposition. We omit the details since the result is now also an immediate consequence of our bijection, Theorem 7.5, along with (1.1).

Proposition 6.3. Fix $n \geq 1$ and a shape $\lambda$ with $l(\lambda) \leq n$. Then for each $d \geq 1$, $\mathcal{M}^{d}(\lambda, n)$ generates $\operatorname{sp}_{2 n}(\lambda ; x)$.

\section{The Symplectic JeU De TAQUin}

In this section we present our main results: the symplectic jeu de taquin algorithm (sjdt-algorithm) and a solution to Problem 6.2. The proofs of these results appear in Sections 9 and 10.

A skew tableau $\mathcal{T}=\left\{\mathbf{t}_{i j}\right\}$ of shape $\lambda \backslash \mu$ is punctured if one of its boxes, say $(p, q) \in \lambda \backslash \mu$, is left empty. This empty box is called the puncture. A punctured signed tableau is semistandard if its entries (ignoring the puncture) weakly increase across the rows and strictly increase down the columns. A semistandard punctured column is admissible if its entries correspond to the circles of an admissible circle diagram. The split-column form of $\mathcal{T}$ is constructed as before, leaving the adjacent boxes $(p, q)_{l}$ and $(p, q)_{r}$ empty. If this split-column form is semistandard then the punctured tableau $\mathcal{T}$ is admissible. The tableaux in the example below are admissible punctured skew tableaux of shape $\left(3^{4}, 2\right) \backslash\left(1^{2}\right)$ with punctures at $(1,2),(2,2)$, and $(4,2)$ respectively.

Fix a shape $\mu$. A box $(x, y) \in \mu$ is an inner corner of $\mu$ if $\mu-\{(x, y)\}$ is a legal shape. A box $(w, z) \notin \mu$ is an outer corner of $\mu$ if $\mu+\{(w, z)\}$ is a legal shape. The sjdt-algorithm is performed on a skew tableau $\mathcal{T} \in \mathcal{D}(\lambda \backslash \mu)$, where $\mu$ is not the empty shape. The algorithm moves a puncture from an inner corner $(x, y)$ of 
$\mu$ to an inner corner $(w, z)$ of $\lambda$. To move the puncture, the sjdt-algorithm repeats one of two steps: The puncture moves one box down (a vertical move) or one box to the right (a horizontal move). We precede the formulation of the sjdt-algorithm with a description of these moves.

Suppose $\mathcal{T}$ is an admissible punctured skew tableau of shape $\lambda \backslash \mu$ with puncture at $(p, q)$. The sjdt-algorithm compares the entry $l_{p, q+1}$ with the entry $r_{p+1, q}$. If $r_{p+1, q} \leq l_{p, q+1}$ or $(p, q+1) \notin \lambda$ then we slide the pair $(l, r)_{p+1, q}$ up into the puncture. For example, since in the tableau at left below $r_{2,2}=\overline{\mathbf{5}}=l_{1,3}$, the puncture moves down under the sjdt-algorithm. The last tableau shows the result after two more vertical moves.

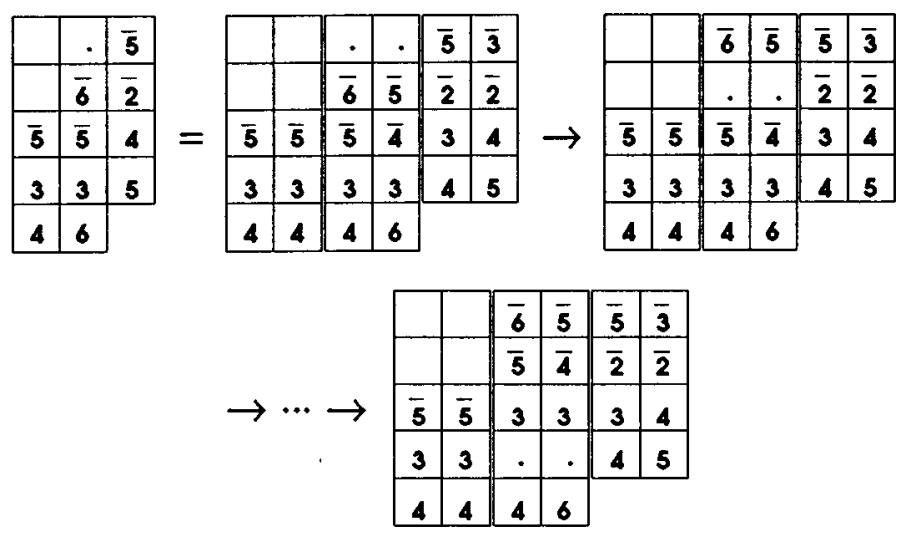

If, on the other hand, $r_{p+1, q}>l_{p, q+1}$ or $(p+1, q) \in \lambda$, a horizontal move is made. Denote the columns of $T$ by $\mathcal{T}_{1}, \mathcal{T}_{2}, \ldots, \mathcal{T}_{c}$ and denote the associated subsets for $\mathcal{T}_{i}$ by $A_{i}, B_{i}, C_{i}, D_{i}$. Subroutine 7.1 moves the puncture to the right:

Subroutine $7.1\left(\mathbf{h}_{R}\right)$.

Input: A punctured admissible skew tableau $\mathcal{T}$ of shape $\lambda \backslash \mu$ with puncture at $(p, q)$ and such that either $(p+1, q)$ is empty or $r_{p+1, q}>l_{p, q+1}$.

If $l_{p, q+1}=\overline{\mathbf{a}}$ (a barred entry) then

Replace $\mathcal{T}_{q}$ by $G\left(B_{q}+\{a\}, C_{q}\right)$ and $\mathcal{T}_{q+1}$ by $F\left(A_{q+1}-\{a\}, D_{q+1}\right)$, inserting empty boxes so that $\mathcal{T}$ remains of shape $\lambda \backslash \mu$ with the puncture now at $(p, q+1)$ :

else $l_{p, q+1}=\mathbf{a}$ (an unbarred entry)

Replace $\mathcal{T}_{q}$ by $F\left(A_{q}, D_{q}+\{a\}\right)$ and $\mathcal{T}_{q+1}$ by $G\left(B_{q+1}, C_{q+1}-\{a\}\right)$, inserting empty boxes as above.

Output: A punctured skew tableaux $\mathcal{T}^{\prime}=\mathbf{h}_{R}(\mathcal{T},(p, q))$ of shape $\lambda \backslash \mu$ with puncture at $(p, q+1)$, with admissible columns, and such that either $(p-1, q+1)$ is empty or $l_{p-1, q+1}^{\prime}<r_{p, q}^{\prime}$.

Note that we do not claim that $\mathbf{h}_{R}(\mathcal{T},(p, q))$ is admissible. It is not hard to produce an admissible punctured skew tableau $\mathcal{T}$ such that the split-column form of $\mathcal{T}^{\prime}=\mathbf{h}_{R}(\mathcal{T},(p, q))$ is not even semistandard. It will be shown, however, that such a $\mathcal{T}$ cannot be the result of any step of the sjdt-algorithm. We defer this issue and all other well definedness issues of the $\mathbf{h}_{R}$ subroutine and the sjdt-algorithm until Section 9. We left the example above with the empty box at $(4,2)$. Since 
$\mathbf{6}=r_{5,2}>l_{4,3}=\mathbf{4}$, the sjdt-algorithm requires us to replace $\mathcal{T}$ with $\mathbf{h}_{R}(\mathcal{T},(4,2))$. We have

$$
\begin{aligned}
& A_{2}=\{5,6\}, \quad B_{2}=\{4,5\}, \quad A_{3}=\{2,5\}, \quad B_{3}=\{2,3\}, \\
& C_{2}=\{3,4\}, \quad D_{2}=\{3,6\}, \quad C_{3}=\{3,4\}, \quad D_{3}=\{4,5\} .
\end{aligned}
$$

We take the 4 from $C_{3}$ and put it in $D_{2}$. Then we replace $\mathcal{T}_{2}$ with $F(\{5,6\},\{3,4,6\})$ and replace $\mathcal{T}_{3}$ with $G(\{2,3\},\{3\})$ :

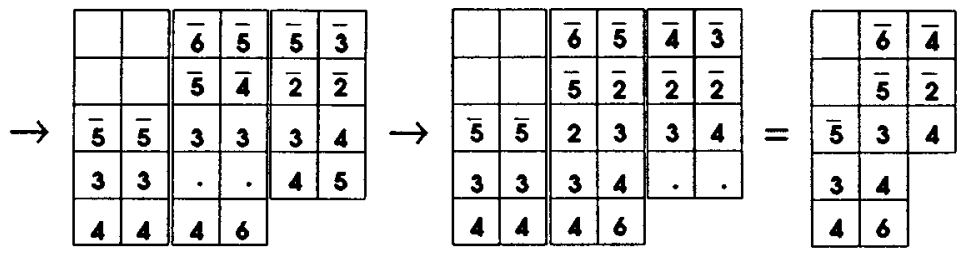

Note that if we focus on the region of the split-column tableau around the boxes $(p, q)_{r}$ and $(p, q+1)_{l}$ (i.e. the regions corresponding to $D_{2}$ and $C_{3}$ in the example above), the horizontal move looks like that of the original jeu de taquin: It is not hard to see that $\mathbf{h}_{R}$ slides the entry $l_{p, q+1}$ of $\mathcal{T}$ to the left to produce the entry $r_{p, q}^{\prime}$ of $\mathbf{h}_{R}(\mathcal{T},(p, q))$. This is because the other entries in $r \mathcal{T}_{q}$ and $l \mathcal{T}_{q+1}$ of the same type as $l_{p, q+1}$ (i.e., barred or unbarred) remain unchanged.

Let $\mathbf{v}_{D}(\mathcal{T},(p, q))$ denote the punctured tableau resulting from a vertical move. The operator $\Pi$ is defined on pairs $(\mathcal{T},(p, q))$ where the puncture $(p, q)$ is not an inner corner of $\lambda$ :

$$
\Pi(\mathcal{T},(p, q))= \begin{cases}\left(\mathbf{v}_{D}(\mathcal{T},(p, q)),(p+1, q)\right), & \text { if } r_{p+1, q} \leq l_{p, q+1} \text { or }(p, q+1) \notin \lambda, \\ \left(\mathbf{h}_{R}(\mathcal{T},(p, q)),(p, q+1)\right), & \text { if } r_{p+1, q}>l_{p, q+1} \text { or }(p+1, q) \notin \lambda .\end{cases}
$$

The sjdt-algorithm is a repeated application of $\Pi$ :

Algorithm 7.2 (sjdt).

Input: $\mathcal{T} \in \mathcal{D}(\lambda, \mu)$ and an inner corner $(x, y)$ of $\mu$.

Set $\mu^{\prime}:=\mu-\{(x, y)\}$ and set $(p, q):=(x, y)$.

While $(p, q)$ is not an inner corner of $\lambda$ repeat the following step: Set $(\mathcal{T},(p, q)):=\Pi(\mathcal{T},(p, q))$.

Set $(w, z):=(p, q)$ and $\lambda^{\prime}:=\lambda-\{(w, z)\}$.

Output: $\mathcal{T} \in \mathcal{D}\left(\lambda^{\prime} \backslash \mu^{\prime}\right)$ and an outer corner $(w, z)$ of $\lambda^{\prime}$.

In the following example the sjdt-algorithm transforms a tableau from $\mathcal{D}\left(\left(2^{2}, 1\right) \backslash\right.$ $(1), 2)$ into a tableau in $\mathcal{D}\left(\left(2,1^{2}\right), 3\right)$ :

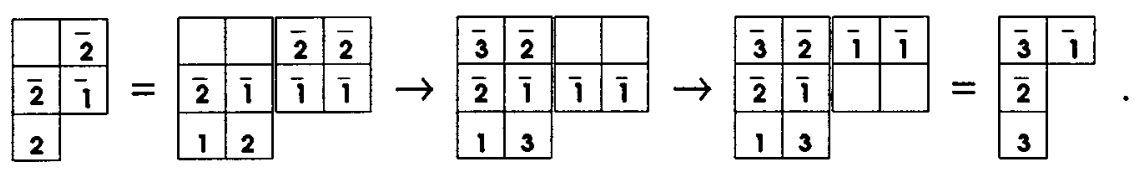

The first main result of this paper states that the sjdt-algorithm is well defined and lists some of its properties. Given the pair $(\mathcal{T},(x, y)) \in \mathcal{D}(\lambda \backslash \mu) \times$ $\{$ inner corners of $\mu\}$ as the input to the algorithm, denote the output by $\operatorname{sjdt}(\mathcal{T},(x, y)) \in \mathcal{D}\left(\lambda^{\prime} \backslash \mu^{\prime}\right) \times\left\{\right.$ outer corners of $\left.\lambda^{\prime}\right\}$. 
Theorem 7.3. The sjdt-algorithm is well defined and provides a bijection

$$
\begin{aligned}
\text { sjdt : } \bigcup_{\lambda \backslash \mu} \mathcal{D}(\lambda \backslash \mu) \times\{\text { inner corners of } \mu\} & \\
& \rightarrow \bigcup_{\lambda \backslash \mu} \mathcal{D}(\lambda \backslash \mu) \times\{\text { outer corners of } \lambda\} .
\end{aligned}
$$

Fix $n \geq 1$ and let $(\mathcal{T},(x, y)) \in \mathcal{D}(\lambda \backslash \mu, n) \times\{$ inner corners of $\mu\}$. Set $(\mathcal{S},(w, z)):=$ $\operatorname{sjdt}(\mathcal{T},(x, y))$. Let $\lambda^{\prime} \backslash \mu^{\prime}$ be the shape of $\mathcal{S}$. Then:

(1) $\lambda^{\prime}=\lambda-\{(w, z)\}, \mu^{\prime}=\mu-\{(x, y)\}$ and $\operatorname{wt}(\mathcal{S})=\operatorname{wt}(\mathcal{T})$.

(2) Suppose $\left(x^{\prime}, y^{\prime}\right)$ is an inner corner of $\mu^{\prime}$ and $\left(\mathcal{R},\left(w^{\prime}, z^{\prime}\right)\right)=\operatorname{sjdt}\left(\mathcal{S},\left(x^{\prime}, y^{\prime}\right)\right)$. If $y^{\prime}<y$ then $z^{\prime}<z$. If $y^{\prime} \geq y$ then $z^{\prime} \geq z$. In either case, the paths the two punctures take as the sjdt-algorithm is applied never cross.

(3) $\mathcal{S} \in \mathcal{D}\left(\lambda^{\prime} \backslash \mu^{\prime}, n+1\right)$. If an $\mathbf{n}+\mathbf{1}$ or $\overline{\mathbf{n}+\mathbf{1}}$ appears in $\mathcal{S}$, then each of these two symbols will appear exactly once in the yth column and nowhere else.

(4) Suppose the symbols $\mathbf{n}+\mathbf{1}$ and $\overline{\mathbf{n + 1}}$ appear in the unsplit form of $\mathcal{S}$. Form the tableau $\mathcal{S}^{\prime} \in \mathcal{D}(\nu \backslash \mu, n)$ by removing these symbols from their boxes. Set $\left(\mathcal{R}^{\prime},\left(w^{\prime \prime}, z^{\prime \prime}\right)\right)=\operatorname{sjdt}\left(\mathcal{S}^{\prime},(x, y)\right)$. Then $z^{\prime \prime}<z$. Moreover, the paths the two punctures take as the sjdt-algorithm is applied never cross.

We now make precise what it means for two paths to cross. The path the puncture takes during an application of sjdt is the sequence of boxes the puncture occupies as the algorithm progresses. We compare only paths arising in two successive applications of sjdt: the first and the second path. Suppose both paths intersect column $q$. Let $\left(i_{\boldsymbol{x}}, q\right)$ (respectively, $\left(i_{\boldsymbol{x}^{\prime}}, q\right)$ ) be the upper most box of column $q$ occurring in the first (respectively, second) path. Let $\left(i_{\boldsymbol{z}}, q\right)$ (respectively, $\left(i_{\boldsymbol{z}^{\prime}}, q\right)$ ) be the lower most box of column $q$ occurring in the first (respectively, second) path. The two paths cross in column $q$ if either: $i_{\boldsymbol{x}^{\prime}} \geq i_{\boldsymbol{x}}$ and $i_{\boldsymbol{z}^{\prime}}<i_{\boldsymbol{z}}$; or $i_{\boldsymbol{x}^{\prime}}<i_{\boldsymbol{x}}$ and $i_{\boldsymbol{z}^{\prime}} \geq i_{\boldsymbol{z}}$.

Part (3) of the theorem implies that an $\mathbf{n}+\mathbf{1}$ and $\overline{\mathbf{n}+\mathbf{1}}$ may appear in $\mathcal{S}=$ $\operatorname{sjdt}(\mathcal{T},(x, y))_{1}$ even though the entries of $\mathcal{T}$ were from $[[\mathbf{n}]]$. If they do appear, they were created by the "column repairs" made during an application of $\mathbf{h}_{R}$. These repairs mark the essential difference between the original jeu de taquin algorithm and the sjdt-algorithm. They also make well definedness a major issue for the sjdtalgorithm. In contrast, the well definedness of sliding out one box of $\mu$ using the original jeu de taquin algorithm is almost obvious.

Our second main result provides for a solution to the tableau bijection problem. Fix $n \geq 1$, let $\lambda$ be a shape with at most $n$ rows, and let $\mathcal{T} \in \mathcal{M}^{n}(\lambda, n)$. Define $\Phi_{\lambda}^{n}(\mathcal{T})$ to be the result of applying the Algorithm 7.4 below to $\mathcal{T}$. Here all admissible tableaux are in their unsplit form.

Algorithm $7.4\left(\Phi_{\lambda}^{n}\right)$.

Input: $\mathcal{T} \in \mathcal{M}^{n}(\lambda, n)$.

Remove all the $\overline{\mathbf{n}}$ 's and $\mathbf{n}$ 's from $\mathcal{T}$ to produce a skew tableau $\mathcal{D} \in \mathcal{M}^{n-1}(\pi \backslash$ $(k), n-1)$.

Let $\mathcal{K}$ be the skew tableau of shape $\lambda \backslash \pi$, each entry of which is $\mathbf{n}$.

While $k \neq 0$ repeat the following steps:

Set $(\mathcal{D},(s, t)):=\operatorname{sjdt}(\mathcal{D},(1, k))$. Place an $\overline{\mathbf{n}}$ in $\mathcal{K}$ at $(s, t)$. 
If there is an $\mathbf{n}$ and an $\overline{\mathbf{n}}$ in (the $k$ th-column of) the new $\mathcal{D}$ then

Take the $\mathbf{n}$ from its box in $\mathcal{D}$ and place it in the corresponding box in $\mathcal{K}$.

Remove the $\overline{\mathbf{n}}$ from $\mathcal{D}$ leaving the box $(1, k)$ empty.

else Set $k:=k-1$.

Output: A tableau $\mathcal{Q} \in \mathcal{M}^{n-1}(\lambda, n)$ with De Concini-part $\mathcal{D}$ and King-part $\mathcal{K}$.

There are several well definedness issues which will be addressed in Section 10. The following result solves Problem 6.2, the reduced bijection problem. As described in that reduction, it provides the heart of our bijection between $\mathcal{D}(\lambda, n)$ and $\mathcal{K}(\lambda, n)$.

Theorem 7.5. For all $n \geq 1$ and shapes $\lambda$ with at most $n$ rows, the map $\Phi_{\lambda}^{n}$ : $\mathcal{M}^{n}(\lambda, n) \rightarrow \mathcal{M}^{n-1}(\lambda, n)$ is a well defined weight preserving bijection.

Below we show the transformation of a tableau in $\mathcal{M}^{3}\left(\left(2^{3}\right), 3\right)$ to a tableau in $\mathcal{M}^{2}\left(\left(2^{3}\right), 3\right)$ as Algorithm 7.4 is applied.

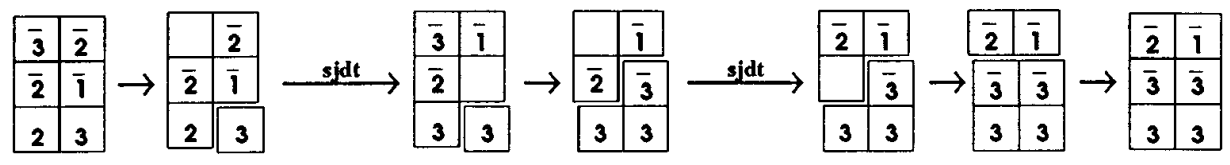

The tableaux $\mathcal{D}$ and $\mathcal{K}$ of the algorithm are shown offset from one another. Note that $\mathcal{D}$ begins as the admissible skew tableau considered in the example preceding Theorem 7.3. The image of the input tableau under the full bijection $\mathcal{D}\left(\left(2^{3}\right), 3\right) \rightarrow$ $\mathcal{K}\left(\left(2^{3}\right), 3\right)$ will be the same as the output tableau above, but with the $\overline{\mathbf{2}}$ and the $\overline{\mathbf{1}}$ interchanged.

\section{Technical Lemmas}

In Section 9 one of our main goals is to show that during the sjdt-algorithm the output tableau of a horizontal move is punctured admissible. View an admissible column, with associated subsets $A, B, C, D$, as a pair of circle diagrams: one admissible for $F(A, D)$, the other coadmissible for $F(B, C)$. The horizontal move begins by moving one entry $\boldsymbol{x}$ from the column $l \mathcal{T}_{q+1}$ to the column $r \mathcal{T}_{q}$. If $\boldsymbol{x}$ is unbarred, this move consists of removing one circle from the coadmissible circle diagram for $\mathcal{T}_{q+1}$ and inserting it into the corresponding box of the admissible circle diagram for $\mathcal{T}_{q}$. If $\boldsymbol{x}$ is barred, swap "admissible" and "coadmissible" in this statement. We now consider when adding a circle to an admissible circle diagram produces an admissible circle diagram.

Fix $n \geq 1$ and let $\mathcal{P}$ be a $2 \times n$ admissible circle diagram with associated subsets $A_{\mathcal{P}}, B_{\mathcal{P}}, C_{\mathcal{P}}, D_{\mathcal{P}}$. An important set is $U_{\mathcal{P}}=A_{\mathcal{P}} \cup B_{\mathcal{P}} \cup C_{\mathcal{P}} \cup D_{\mathcal{P}}$, the set of all slots in $\mathcal{P}$ which are used in some way. Note we also have $U_{\mathcal{P}}=A_{\mathcal{P}}+C_{\mathcal{P}}=B_{\mathcal{P}}+D_{\mathcal{P}}=$ $\left(A_{\mathcal{P}} \cup D_{\mathcal{P}}\right)+J_{\mathcal{P}}=\left(B_{\mathcal{P}} \cup C_{\mathcal{P}}\right)+I_{\mathcal{P}}$. If $U_{\mathcal{P}}=[n]$ then there is no way to add a circle to $\mathcal{P}$ and maintain admissiblity: If $U_{\mathcal{P}}=[n]$, then all of the empty slots are listed in $J_{\mathcal{P}}$, i.e., $J_{\mathcal{P}}=H_{\mathcal{P}}$. Adding a circle to $\mathcal{P}$ either destroys an empty slot or creates a full slot, resulting in more full slots than empty slots.

Lemma 8.1. Fix $n \geq a \geq 1$ and let $\mathcal{R}$ be $a 2 \times n$ admissible circle diagram. Suppose $U_{\mathcal{R}}=\{2,3, \ldots, n\}$ and suppose the ath slot of $\mathcal{R}$ is either empty or half-full. Add a circle to the ath slot of $\mathcal{R}$ producing a circle diagram $\mathcal{P}$. Then $\mathcal{P}$ is admissible. 
If the ath slot of $\mathcal{R}$ is empty then $I_{\mathcal{P}}=I_{\mathcal{R}}$ and $J_{\mathcal{P}}=\left(J_{\mathcal{R}}+\{1\}\right)-\{a\}$. If the ath slot of $\mathcal{R}$ is half-full then $I_{\mathcal{P}}=I_{\mathcal{R}}+\{a\}$ and $J_{\mathcal{P}}=J_{\mathcal{R}}+\{1\}$.

Proof. Note that all the empty slots of $\mathcal{R}$ other than the first are listed by $J_{\mathcal{R}}$. The result is clear if $a=1$ so assume $a>1$. Suppose the $a$ th slot is listed in $J_{\mathcal{R}}$. Then there are exactly as many empty slots in $\mathcal{P}$ as full slots. These empty slots are listed by $J_{\mathcal{P}}=\left(J_{\mathcal{R}}+\{1\}\right)-\{a\}$. Clearly $J_{\mathcal{P}} \leq J_{\mathcal{R}}$. Since $J_{\mathcal{R}}<I_{\mathcal{R}}=I_{\mathcal{P}}, \mathcal{P}$ is admissible. If the ath slot of $\mathcal{R}$ is half-full then that of $\mathcal{P}$ is full: $I_{\mathcal{P}}=I_{\mathcal{R}}+\{a\}$. In $\mathcal{P}$, there are again exactly as many empty slots as full slots. The empty slots are now listed by $J_{\mathcal{P}}=J_{\mathcal{R}}+\{1\}$. Since $J_{\mathcal{R}}<I_{\mathcal{R}}$ and $a>1$, we have $J_{\mathcal{R}}+\{1\} \leq I_{\mathcal{R}}+\{a\}$. So again $\mathcal{P}$ is admissible.

Let $\mathcal{Q}$ be a $2 \times n$ admissible circle diagram. The slots in $[n]-U_{\mathcal{Q}}$ are the unused slots of $\mathcal{Q}$. In order to apply Lemma 8.1, we partition $\mathcal{Q}$ into contiguous admissible parts. Each of these parts (except for possibly the leftmost) will satisfy the condition on $\mathcal{R}$ of Lemma 8.1. For any $2 \times n$ circle diagram $\mathcal{P}$ and for any interval $S$ of $[n]$ denote the $\mathcal{P}_{S}$ the smaller circle diagram consisting only of those slots of $\mathcal{P}$ listed in $S$. If $\mathcal{P}_{S}$ is admissible, denote the associated subsets by $A_{\mathcal{P}, S}, B_{\mathcal{P}, S}, C_{\mathcal{P}, S}, D_{\mathcal{P}, S}$. Similarly use the notation $I_{\mathcal{P}, S}, J_{\mathcal{P}, S}, H_{\mathcal{P}, S}, U_{\mathcal{P}, S}$.

Lemma 8.2. Fix $n \geq 1$. Let $\mathcal{Q}$ be a $2 \times n$ admissible circle diagram. Let $\left\{b_{1}<\right.$ $\left.b_{2}<\cdots<b_{k}\right\}$ list the unused slots of $\mathcal{Q}$. Set $b_{0}=1$ and $b_{k+1}=n+1$.

(1) The circle diagram $\mathcal{Q}_{\left[b_{i}, b_{i+1}\right)}$ is admissible for $0 \leq i \leq k$.

(2) For all $\left(l_{m}, r_{m}\right)$ in the split-column form of $\mathcal{Q}$, we have $b_{i}<\left\|l_{m}\right\|<b_{i+1}$ if and only if $b_{i}<\left\|r_{m}\right\|<b_{i+1}, 0 \leq i \leq k$.

Add a circle to the ath slot of $\mathcal{Q}$ (which is either empty or half-full) producing a circle diagram $\mathcal{P}$.

(3) $\mathcal{P}$ is admissible if and only if $a \in\left[b_{1}, n\right]$.

(4) If $a \in\left[b_{j}, b_{j+1}\right)$ and the ath slot of $\mathcal{Q}$ was empty then $I_{\mathcal{P}}=I_{\mathcal{Q}}$ and $J_{\mathcal{P}}=$ $\left(J_{\mathcal{Q}}+\left\{b_{j}\right\}\right)-\{a\} ;$ if the ath slot of $\mathcal{Q}$ was half-full then $I_{\mathcal{P}}=I_{\mathcal{R}}+\{a\}$ and $J_{\mathcal{P}}=J_{\mathcal{R}}+\left\{b_{j}\right\}$.

Proof. We start with Part (1). It follows directly from the definition of admissibility that for any $i, 1 \leq i \leq n$, the circle diagram $\mathcal{Q}_{[1, i)}$ is admissible. We show that $\mathcal{Q}_{\left[b_{j}, n\right]}$ is admissible for $1 \leq j \leq k$. The first sentence will then imply that $\mathcal{Q}_{\left[b_{j}, b_{j+1}\right)}$ is admissible for each $j<k$. Fix some such $j$. To show that $\mathcal{Q}_{\left[b_{j}, n\right]}$ is admissible it is sufficient to show that $I_{\mathcal{Q},\left[b_{j} n\right]}<J_{\mathcal{Q},\left[b_{j} n\right]}$. Let $\left\{\kappa_{1}<\kappa_{2}<\cdots \kappa_{s}\right\}$ be the elements of $I_{\mathcal{Q}}$ and let $\left\{\gamma_{1}<\gamma_{2}<\cdots<\gamma_{s}\right\}$ be the elements of $J_{\mathcal{Q}}$. We claim that there exists a largest $m$ such that $\gamma_{m}<\kappa_{m}<b_{j}$ and if $m<s$ then $b_{j}<\gamma_{m+1}<\kappa_{m+1}$. Suppose not. Then there exists an $i$ such that $\gamma_{i}<b_{j}<\kappa_{i}$. Form the set $J^{\prime}:=\left(J_{\mathcal{Q}}-\gamma_{i}\right)+b_{j}$. It follows that $J^{\prime} \subseteq H_{\mathcal{Q}}$, with $J_{\mathcal{Q}}<J^{\prime}<I_{\mathcal{Q}}$. This contradicts the definition of $J_{\mathcal{Q}}$. Thus, $I_{\mathcal{Q},\left[b_{j} n\right]}=\left\{\gamma_{m+1}, \ldots, \gamma_{s}\right\}<\left\{\kappa_{m+1}, \ldots, \kappa_{s}\right\}=J_{\mathcal{Q},\left[b_{j} n\right]}$ and Part (1) follows. Parts (3) and (4) now follow easily from (1) and Lemma 8.1 since all of the circle diagrams $\mathcal{Q}_{\left[b_{1}, b_{2}\right)}, \mathcal{Q}_{\left[b_{2}, b_{3}\right)}, \ldots, \mathcal{Q}_{\left[b_{k}, n\right]}$ satisfy the conditions of $\mathcal{R}$ in that lemma.

Now for Part (2). Let $t=\left|A_{\mathcal{Q},\left[1, b_{1}\right)}\right|$. Then for $1 \leq m \leq t$ we have $1 \leq\left\|l_{m}\right\|<b_{1}$, and if $l_{t+1}$ is barred then $\left\|l_{t+1}\right\| \geq b_{1}$. The same is true for the entries $r_{1}, \ldots, r_{t}$ and $r_{t+1}$ since $\left|B_{\mathcal{Q},\left[1, b_{1}\right)}\right|=\left|A_{\mathcal{Q},\left[1, b_{1}\right)}\right|$. Since $\left|B_{\mathcal{Q},\left[b_{1}, b_{i+1}\right)}\right|=\left|A_{\mathcal{Q},\left[b_{1}, b_{i+1}\right)}\right|$ for $0 \leq i \leq k$, an easy induction argument on $i$ verifies the claim for all the barred entries of $l \mathcal{Q}$ and $r \mathcal{Q}$. A similar argument using $\left|D_{\mathcal{Q},\left[b_{i}, b_{i+1}\right)}\right|=\left|C_{\mathcal{Q},\left[b_{i}, b_{i+1}\right)}\right|$ applies to the unbarred entries. 
In order to describe the result of a horizontal move we must also consider adding circles to coadmissible circle diagrams. Further, we must distinguish adding a circle to the top row of a circle diagram from adding a circle to the bottom row. We do this by appealing to symmetry.

Fix $n \geq k \geq 1$. Our goal is to define an action of the Klein four-group on the set $\mathcal{D}\left(\left(1^{k}\right), n\right)$. We start with a simpler action of the Klein four-group on the set of all $2 \times n$ circle diagrams. Let this first action be generated by the horizontal reflection $\tau^{\prime}$ defined in Section 5 and the obvious vertical reflection, which we denote by $\sigma$. For example,

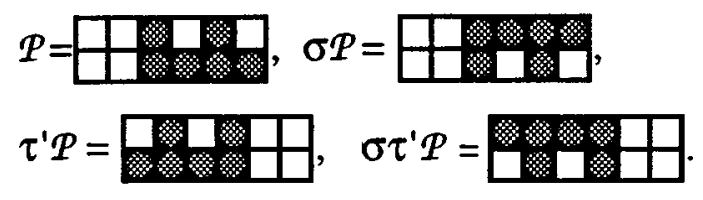

For $x \in[n]$ denote the integer $n+1-x$ by $x^{\vee}$. For any subset $X \subset[n]$ denote the set $\{n-x+1: x \in X\}$ by $X^{\vee}$. Suppose $\mathcal{P}$ corresponds to a semistandard column with $\mathcal{P}=F(X, Y)$. The action above induces an action on columns given by $\sigma \mathcal{P}=F(Y, X), \tau^{\prime} \mathcal{P}=F\left(X^{\vee}, Y^{\vee}\right)$.

Let $\mathcal{P} \in \mathcal{D}\left(\left(1^{k}\right), n\right)$ have associated subsets $A_{\mathcal{P}}, B_{\mathcal{P}}, C_{\mathcal{P}}, D_{\mathcal{P}}$. Then $\sigma \mathcal{P} \in$ $\mathcal{D}\left(\left(1^{k}\right), n\right)$ has associated subsets $A_{\sigma \mathcal{P}}=D_{\mathcal{P}}, B_{\sigma \mathcal{P}}=C_{\mathcal{P}}, C_{\sigma \mathcal{P}}=B_{\mathcal{P}}, D_{\sigma \mathcal{P}}=A_{\mathcal{P}}$. We know that $\tau^{\prime} \mathcal{P}=F\left(A_{\mathcal{P}}^{\vee}, D_{\mathcal{P}}^{\vee}\right)$ corresponds to a coadmissible $2 \times n$ circle diagram. Now define $\tau \mathcal{P}:=G\left(A_{\mathcal{P}}^{\vee}, D_{\mathcal{P}}^{\vee}\right)$. Then $\tau \mathcal{P}$ is admissible and has associated subsets $A_{\tau \mathcal{P}}=B_{\mathcal{P}}^{\vee}, B_{\tau \mathcal{P}}=A_{\mathcal{P}}^{\vee}, C_{\tau \mathcal{P}}=D_{\mathcal{P}}^{\vee}, D_{\tau \mathcal{P}}=C_{\mathcal{P}}^{\vee}$. Thus, for fixed $n \geq k \geq 1$, the maps $\sigma$ and $\tau$ generate a Klein four-group, $K_{4}:=\{\mathrm{id}, \sigma, \tau, v:=\sigma \cdot \tau\}$, which acts on $\mathcal{D}\left(\left(1^{k}\right), n\right)$. The action on the split-column form is given schematically below:

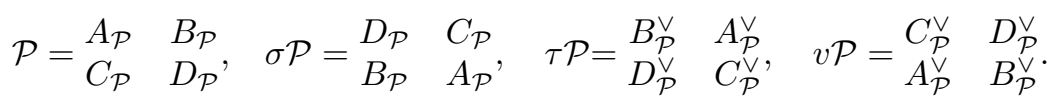

Lemma 8.3 below provides for a precise description of the outcome of a horizontal move. The lemma compares two columns $\mathcal{P}$ and $\mathcal{Q}$. To simplify its statement, the lemma is written from the opposite (yet equivalent) point of view taken above: here we are removing a circle from the circle diagram for $\mathcal{P}$ to get that of $\mathcal{Q}$. Suppose that during an application of $\mathbf{h}_{R}$ to a punctured skew tableau $\mathcal{T}$, the puncture moves from column $q$ to column $q+1$. While reading part (1) or part (3) of the lemma, think of $\mathcal{P}$ as corresponding to column $q+1$ of $\mathcal{T}$ before the puncture arrives and think of $\mathcal{Q}$ as corresponding to the same column after the puncture arrives. For parts (2) and (4), take $\mathcal{Q}$ and $\mathcal{P}$ to respectively correspond to column $q$ before and after the puncture leaves. Given a semistandard column $\mathcal{P}=\left\{p_{i}\right\}_{1 \leq i \leq m}$, define $\operatorname{index}\left[p_{k}, \mathcal{P}\right]=k$.

Lemma 8.3. Let $\mathcal{P} \in \mathcal{D}\left(\left(1^{k}\right), n\right)$ and $\mathcal{Q} \in \mathcal{D}\left(\left(1^{k-1)}, n\right)\right.$ be admissible columns with associated subsets $\left(A_{\mathcal{P}}, B_{\mathcal{P}}, C_{\mathcal{P}}, D_{\mathcal{P}}\right)$ and $\left(A_{\mathcal{Q}}, B_{\mathcal{Q}}, C_{\mathcal{Q}}, D_{\mathcal{Q}}\right)$ respectively. Let $U_{\mathcal{Q}}=A_{\mathcal{Q}} \cup B_{\mathcal{Q}} \cup C_{\mathcal{Q}} \cup D_{\mathcal{Q}}$.

(1) If $A_{\mathcal{Q}}=A_{\mathcal{P}}-\{a\}$ and $D_{\mathcal{Q}}=D_{\mathcal{P}}$, set $b=\max \left[\{1,2, \ldots, a\} \backslash U_{\mathcal{Q}}\right]$. Then

(i) $B_{\mathcal{Q}}=B_{\mathcal{P}}-\{b\}$ and $C_{\mathcal{Q}}=\left(C_{\mathcal{P}}+\{a\}\right)-\{b\}$; and

(ii) index $[\overline{\mathbf{a}}, l \mathcal{P}] \leq$ index $[\overline{\mathbf{b}}, r \mathcal{P}]$.

(2) If $B_{\mathcal{Q}}=B_{\mathcal{P}}-\{a\}$ and $C_{\mathcal{Q}}=C_{\mathcal{P}}$, set $b=\min \left[\{n, n-1, \ldots, a\} \backslash U_{\mathcal{Q}}\right]$. Then

(i) $A_{\mathcal{Q}}=A_{\mathcal{P}}-\{b\}$ and $D_{\mathcal{Q}}=\left(D_{\mathcal{P}}+\{a\}\right)-b$; and 
(ii) index $[\overline{\mathbf{b}}, l \mathcal{P}] \leq \operatorname{index}[\overline{\mathbf{a}}, r \mathcal{P}]$.

(3) If $C_{\mathcal{Q}}=C_{\mathcal{P}}-\{a\}$ and $B_{\mathcal{Q}}=B_{\mathcal{P}}$, set $b=\min \left[\{n, n-1, \ldots, a\} \backslash U_{\mathcal{Q}}\right]$. Then

(i) $D_{\mathcal{Q}}=D_{\mathcal{P}}-\{b\}$ and $A_{\mathcal{Q}}=\left(A_{\mathcal{P}}+\{a\}\right)-\{b\}$; and

(ii) index $[\mathbf{a}, l \mathcal{P}] \leq$ index $[\mathbf{b}, r \mathcal{P}]$.

(4) If $D_{\mathcal{Q}}=D_{\mathcal{P}}-\{a\}$ and $A_{\mathcal{Q}}=A_{\mathcal{P}}$, set $b=\max \left[\{1,2, \ldots, a\} \backslash U_{\mathcal{Q}}\right]$. Then

(i) $C_{\mathcal{Q}}=C_{\mathcal{P}}-\{b\}$ and $B_{\mathcal{Q}}=\left(B_{\mathcal{P}}+\{a\}\right)-\{b\}$; and

(ii) index $[\mathbf{b}, l \mathcal{P}] \leq \operatorname{index}[\mathbf{a}, r \mathcal{P}]$.

Proof. First, using $\tau \in K_{4}$, we confirm that (1) implies (2). Suppose (1) is true. Suppose further that we have the conditions of (2): $B_{\mathcal{Q}}=B_{\mathcal{P}}-\{a\}$ and $C_{\mathcal{Q}}=$ $C_{\mathcal{P}}$. For $\mathcal{X}=\mathcal{P}, \mathcal{Q}$, the associated subsets of $\tau \mathcal{X}$ are given by $A_{\tau x}=B_{x}^{\vee}, B_{\tau x}=$ $A_{x}^{\vee}, C_{\tau x}=D_{x}^{\vee}, D_{\tau x}=C_{x}^{\vee}$. Since $A_{\tau \mathcal{Q}}=B_{\mathcal{Q}}^{\vee}=B_{\mathcal{P}}^{\vee}-\left\{a^{\vee}\right\}$ and $D_{\tau \mathcal{Q}}=C_{\mathcal{Q}}^{\vee}=C_{\mathcal{P}}^{\vee}=$ $D_{\tau \mathcal{P}}$ we apply (1): Set $b=\max \left[\left\{1,2, \ldots, a^{\vee}\right\} \backslash U_{\tau \mathcal{Q}}\right]$. Then, (1.i) $B_{\tau \mathcal{Q}}=B_{\tau \mathcal{P}}-\{b\}$ and $C_{\tau \mathcal{Q}}=\left(C_{\tau \mathcal{P}}+\left\{a^{\vee}\right\}\right)-\{b\}$ and (1.ii) index $\left[\overline{\mathbf{a}^{\vee}}, l(\tau \mathcal{P})\right] \leq \operatorname{index}[\overline{\mathbf{b}}, r(\tau \mathcal{P})]$. Now apply the map $X \mapsto X^{\vee}$. Then $b^{\vee}=\min \left[\{n, n-1, \ldots, a\} \backslash U_{\mathcal{Q}}\right]$ since $U_{\tau \mathcal{Q}}=U_{\mathcal{Q}}^{\vee}$. We also have $(2 . \mathrm{i}): A_{\mathcal{Q}}=B_{\tau \mathcal{Q}}^{\vee}=B_{\tau \mathcal{P}}^{\vee}-\left\{b^{\vee}\right\}=A_{\mathcal{P}}-\left\{b^{\vee}\right\}$ and $D_{\mathcal{Q}}=C_{\tau \mathcal{Q}}^{\vee} \stackrel{ }{=}$ $\left(C_{\tau \mathcal{P}}^{\vee}+\{a\}\right)-\left\{b^{\vee}\right\}=\left(D_{\mathcal{P}}+\{a\}\right)-\left\{b^{\vee}\right\}$. For $\left(2\right.$.ii) notice that index $\left[\overline{\mathbf{a}^{\vee}}, l(\tau \mathcal{P})\right] \leq$ index $[\overline{\mathbf{b}}, r(\tau \mathcal{P})] \Leftrightarrow\left|\left\{x \in B_{\mathcal{P}}^{\vee}: x \geq a^{\vee}\right\}\right| \leq\left|\left\{x \in A_{\mathcal{P}}^{\vee}: x \geq b\right\}\right| \Leftrightarrow \mid\left\{x \in B_{\mathcal{P}}: x \leq\right.$ $a\}|\leq|\left\{x \in A_{\mathcal{P}}: x \leq b^{\vee}\right\} \mid \Leftrightarrow \operatorname{index}\left[\overline{\mathbf{b}^{\vee}}, l \mathcal{P}\right] \leq \operatorname{index}[\overline{\mathbf{a}}, r \mathcal{P}]$. Thus, (1) implies (2). Using $\sigma$, it is easier to see that (1) and (4) are equivalent and that (2) and (3) are equivalent.

We prove (1). For (1.i), note that since we are assuming $\mathcal{P}$ is admissible, Lemma $8.2(3)$ implies there exists an unused slot of $\mathcal{Q}$ weakly to the left of $a$. Thus $b$ is well defined. If the $a$ th slot of $\mathcal{Q}$ is empty then Lemma 8.2(4) implies $I_{\mathcal{Q}}=I_{\mathcal{P}}$ and $J_{\mathcal{Q}}=\left(J_{\mathcal{P}}+\{a\}\right)-\{b\}$. Thus

$$
\begin{aligned}
& B_{\mathcal{Q}}=\left(A_{\mathcal{Q}}-I_{\mathcal{Q}}\right)+J_{\mathcal{Q}}=\left(\left(A_{\mathcal{P}}-\{a\}\right)-I_{\mathcal{P}}\right)+\left(\left(J_{\mathcal{P}}+\{a\}\right)-\{b\}\right)=B_{\mathcal{P}}-\{b\}, \text { and } \\
& C_{\mathcal{Q}}=\left(D_{\mathcal{Q}}-I_{\mathcal{Q}}\right)+J_{\mathcal{Q}}=\left(D_{\mathcal{P}}-I_{\mathcal{P}}\right)+\left(\left(J_{\mathcal{P}}+\{a\}\right)-\{b\}\right)=\left(C_{\mathcal{P}}+\{a\}\right)-\{b\} .
\end{aligned}
$$

If the $a$ th slot of $\mathcal{Q}$ is half-full then Lemma 8.2(4) gives $I_{\mathcal{P}}=I_{\mathcal{Q}}-\{a\}$ and $J_{\mathcal{Q}}=$ $J_{\mathcal{P}}-\{b\}$. Thus

$$
\begin{aligned}
& B_{\mathcal{Q}}=\left(A_{\mathcal{Q}}-I_{\mathcal{Q}}\right)+J_{\mathcal{Q}}=\left(\left(A_{\mathcal{P}}-\{a\}\right)-\left(I_{\mathcal{P}}-\{a\}\right)\right)+\left(J_{\mathcal{P}}-\{b\}\right)=B_{\mathcal{P}}-\{b\}, \text { and } \\
& C_{\mathcal{Q}}=\left(D_{\mathcal{Q}}-I_{\mathcal{Q}}\right)+J_{\mathcal{Q}}=\left(D_{\mathcal{P}}-\left(I_{\mathcal{P}}-\{a\}\right)\right)+\left(J_{\mathcal{P}}-\{b\}\right)=\left(C_{\mathcal{P}}+\{a\}\right)-\{b\} .
\end{aligned}
$$

Now for (1.ii): Denote the entries of the split-column form of $\mathcal{P}$ by $\left(l_{i}^{\prime}, r_{i}^{\prime}\right)$ and those of $\mathcal{Q}$ by $\left(l_{i}, r_{i}\right)$. Set $l_{j}^{\prime}=\overline{\mathbf{a}}$ and $r_{m}^{\prime}=\overline{\mathbf{b}}$. If $r_{m}^{\prime}$ is the largest barred entry in $r \mathcal{P}$ then $l_{m}^{\prime}$ is the largest barred entry in $l \mathcal{P}$ and so $j \leq m$. If $r_{m}^{\prime}$ is not the largest barred entry in $r \mathcal{P}$, then $r_{m+1}^{\prime}$ is barred and $\left\|r_{m+1}\right\|<b$. Note that $r_{m+1}^{\prime}=r_{m}$. By Lemma 8.2(2), $\left\|l_{m}\right\|<b$. Now $a \geq b$ implies $j \leq m$.

The reader may wish to skip the remaining lemmas of this section until they are needed in Section 9. Let $\mathcal{P}$ be an admissible column. Given any entry $\boldsymbol{x}$ in $l \mathcal{P}$, parts (1) and (3) of Lemma 8.3 (with $\boldsymbol{x}=\overline{\mathbf{a}}$ and a respectively) specify a unique element $\boldsymbol{y}$ in $r \mathcal{P}$. (Then $\boldsymbol{y}=\overline{\mathbf{b}}$ and $\mathbf{b}$ respectively.) Let $L_{\mathcal{P}}: l \mathcal{P} \rightarrow r \mathcal{P}$ be the map taking $\boldsymbol{x}$ to $\boldsymbol{y}$. Similarly parts (2) and (4) define a map $R_{\mathcal{P}}: r \mathcal{P} \rightarrow l \mathcal{P}$, where $\overline{\mathbf{a}} \mapsto \overline{\mathbf{b}}$ and $\mathbf{a} \mapsto \mathbf{b}$ respectively. If the circle diagram for an admissible column divides into admissible parts, then $L_{\mathcal{P}}(\overline{\mathbf{a}})$ depends only on the part containing $\overline{\mathbf{a}}$ :

Lemma 8.4. Let $\mathcal{P}_{m}$ be a $2 \times m$ admissible circle diagram and let $\mathcal{P}_{n}$ be a $2 \times n$ admissible circle diagram. Form the $2 \times(m+n)$ circle diagram $\mathcal{P}_{m+n}$ by adjoining 
$\mathcal{P}_{m}$ to the left of $\mathcal{P}_{n}$. Consider the corresponding columns $\mathcal{P}_{m}, \mathcal{P}_{n}, \mathcal{P}_{m+n}$. If $\overline{\mathbf{a}}$ is an entry of $l \mathcal{P}_{n}$, then $L_{\mathcal{P}_{n}}(\overline{\mathbf{a}})=\overline{\mathbf{b}}$ if and only if $L_{\mathcal{P}_{m+n}}(\overline{\mathbf{a}+\mathbf{m}})=\overline{\mathbf{b}+\mathbf{m}}$.

Proof. Let $\mathcal{Q}_{n}$ denote the circle diagram for the unsplit column $F\left(A_{\mathcal{P}_{n}}-\{a\}, D_{\mathcal{P}_{n}}\right)$. Then the circle diagram for $\mathcal{Q}_{m+n}:=F\left(A_{\mathcal{P}_{m+n}}-\{a+m\}, D_{\mathcal{P}_{m+n}}\right)$ is formed by adjoining $\mathcal{P}_{m}$ to the left of $\mathcal{Q}_{n}$. Then $U_{\mathcal{Q}_{m+n}}=U_{\mathcal{P}_{m}}+\left\{m+x: x \in U_{\mathcal{Q}_{n}}\right\}$. Thus $b=\max \left[\{1,2, \ldots, a\} \backslash U_{\mathcal{Q}_{n}}\right]$ if and only if $b+m=\max [\{m+1, m+2, \ldots, m+a\} \backslash$ $\left.U_{m+n}\right]$.

There are three statements equivalent to Lemma 8.4 through the action of $K_{4}$ which we omit. One of them is implicitly used in penultimate sentence of the proof of the next lemma. This lemma is needed for the proof of Theorem 7.3(2).

Lemma 8.5. Fix $n \geq k \geq 1$ and an admissible column $\mathcal{P} \in \mathcal{D}\left(\left(1^{k}\right), n\right)$. Then (1) $L_{\mathcal{P}}(\boldsymbol{x}) \geq L_{\mathcal{P}}\left(\boldsymbol{x}^{\prime}\right)$ for all $\boldsymbol{x} \geq \boldsymbol{x}^{\prime} \in l \mathcal{P}$ and $(2) L_{\mathcal{P}}\left(R_{\mathcal{P}}(\boldsymbol{x})\right) \geq \boldsymbol{x}$ for all $\boldsymbol{x} \in r \mathcal{P}$.

Proof. To prove (1), first consider the case where both $\boldsymbol{x}$ and $\boldsymbol{x}^{\prime}$ are barred symbols. Set $\overline{\mathbf{a}}=\boldsymbol{x}$ and $\overline{\mathbf{c}}=\boldsymbol{x}^{\prime}$ with $a \leq c$. Set $\overline{\mathbf{b}}=L_{\mathcal{P}}(\overline{\mathbf{a}})$ and $\overline{\mathbf{d}}=L_{\mathcal{P}}(\overline{\mathbf{c}})$. We must show $\overline{\mathbf{b}} \geq \overline{\mathbf{d}}$, i.e., $b \leq d$. Set $\mathcal{Q}:=F\left(A_{\mathcal{P}}-\{a\}, D_{\mathcal{P}}\right)$. By Lemma 8.3(1) we have $b=\max \left[\{1,2, \ldots, a\} \backslash U_{\mathcal{Q}}\right]$. Thus $b \leq a$ and the $b$ th slot of $\mathcal{Q}$ is unused. If $b=1$ then $b \leq d$. If $b>1$, Lemma 8.2(1) implies $\mathcal{P}$ splits into admissible circle diagrams $\mathcal{P}_{[1, b)}$ and $\mathcal{P}_{[b, n]}$. Since $b \leq a<c$, the circle at $\overline{\mathbf{c}}$ in $\mathcal{P}$ is in the subdiagram $\mathcal{P}_{[b, n]}$. Lemma 8.4 implies that $\overline{\mathbf{d}}$ corresponds to a circle in the coadmissible partner to $\mathcal{P}_{[b, n]}$. Thus $b \leq d$. The case where both $\boldsymbol{x}$ and $\boldsymbol{x}^{\prime}$ are unbarred is equivalent via the action of $v \in K_{4}$. The case where $\boldsymbol{x}$ is unbarred and $\boldsymbol{x}^{\prime}$ is barred is obvious.

To prove (2) consider the case $\boldsymbol{x}$ is a barred element. The unbarred case is equivalent via the action of $v$. Set $\overline{\mathbf{d}}=\boldsymbol{x}, \overline{\mathbf{a}}=R_{\mathcal{P}}(\boldsymbol{x})$ and $\overline{\mathbf{b}}=L_{\mathcal{P}}(\overline{\mathbf{a}})$. We must show $b \leq d$. The argument is as above: The circle diagram for $\mathcal{P}_{[b, n]}$ is admissible. Since $a \geq b, \overline{\mathbf{a}}$ corresponds to a circle in $\mathcal{P}_{[b, n]}$. Since $\overline{\mathbf{a}}=R_{\mathcal{P}}(\overline{\mathbf{d}}), \overline{\mathbf{d}}$ must correspond to a circle in the coadmissible partner to $\mathcal{P}_{[b, n]}$. Thus $b \leq d$.

The last two lemmas of this section will help show that the result of $\mathbf{h}_{R}$ remains admissible punctured during the sjdt-algorithm.

Lemma 8.6. Let $\mathcal{T}=\left\{\mathbf{t}_{i j}\right\}$ be a semistandard skew tableaux with columns $\mathcal{T}_{1}, \mathcal{T}_{2}$, $\ldots, \mathcal{T}_{c}$. Fix $1 \leq q \leq c$ and let $X$ consist of all $\|\boldsymbol{x}\|$ such that $\boldsymbol{x}$ is a barred entry of $\mathcal{T}_{q}$. Suppose the first non-empty box of $\mathcal{T}_{q}$ is at $(h+1, q)$. Then $\mathcal{T}_{q}$ is admissible if and only if for all unbarred entries $\mathbf{t}_{k, q}$ we have $\left\|\mathbf{t}_{\mathbf{k}, \mathbf{q}}\right\| \geq k-\left(h+\left|\left\{x \in X: x>\left\|\mathbf{t}_{k, q}\right\|\right\}\right|\right)$.

Proof. In the circle diagram for $\mathcal{T}_{q}, k-h$ counts all the circles in the top row plus the circles in the bottom row weakly to the left of slot $\left\|\mathbf{t}_{k, q}\right\|$. The expression $\left|\left\{x \in X: x>\left\|\mathbf{t}_{k, q}\right\|\right\}\right|$ counts the circles in the top row strictly to the right of slot $\left\|\mathbf{t}_{k, q}\right\|$. Therefore $k-\left(h+\left|\left\{x \in X: x>\left\|\mathbf{t}_{k, q}\right\|\right\}\right|\right)$ is number of circles contained in the first $\left\|\mathbf{t}_{k, q}\right\|$ slots of the circle diagram for $\mathcal{T}_{q}$. Hence, if $\mathcal{T}_{q}$ is admissible it will satisfy the condition. If the circle diagram for $\mathcal{T}_{q}$ is not admissible then there exists a $b$ such that the $b$ th slot is full and $\left|\left\{\boldsymbol{x} \in \mathcal{T}_{q}:\|\boldsymbol{x}\| \leq b\right\}\right|>b$. The condition then fails for $\mathbf{t}_{k, q}=\mathbf{b}$.

Let $\mathcal{T}=\left\{\mathbf{t}_{i j}\right\}$ be an admissible tableau and fix an entry $\mathbf{t}_{p, q}$ of $\mathcal{T}$. Let $\mathcal{T}^{\prime}$ be the punctured tableau produced by removing entry $\mathbf{t}_{p, q}$. The next lemma implies that $\mathcal{T}^{\prime}$ is admissible. Let $\mathcal{P}$ be a semistandard column and let $\mathcal{Q}$ be a punctured semistandard column. We say $\mathcal{P} \leq \mathcal{Q}$ (or $\mathcal{Q} \leq \mathcal{P}$ ) if $\mathcal{P}$ can sit to the left (or right) of $\mathcal{Q}$ in some punctured semistandard tableau. 
Lemma 8.7. Fix $n \geq k \geq 1$. Consider $\mathcal{P} \in \mathcal{D}\left(\left(1^{k}\right), n\right)$ with associated subsets $A_{\mathcal{P}}, B_{\mathcal{P}}, C_{\mathcal{P}}, D_{\mathcal{P}}$. Let $\left\{(l, r)_{i}\right\}_{1 \leq i \leq k}$ be the entries of the split-column form of $\mathcal{P}$. Fix $1 \leq j \leq k$. Form a punctured column $\mathcal{Q}$ of length $k$ by inserting a puncture at the $j$ th position of:

$$
\begin{aligned}
& F\left(A_{\mathcal{P}}-\{a\}, D_{\mathcal{Q}}\right) \text { or } G\left(B_{\mathcal{P}}-\{c\}, C_{\mathcal{Q}}\right) \text { if }(l, r)_{j}=(\overline{\mathbf{a}}, \overline{\mathbf{c}}) \\
& G\left(B_{\mathcal{P}}, C_{\mathcal{P}}-\{a\}\right) \text { or } F\left(A_{\mathcal{P}}, D_{\mathcal{Q}}-\{c\}\right) \text { if }(l, r)_{j}=(\mathbf{a}, \mathbf{c}) .
\end{aligned}
$$

Then $\mathcal{Q}$ is an admissible punctured column such that $l \mathcal{P} \leq l \mathcal{Q}$ and $r \mathcal{Q} \leq r \mathcal{P}$.

Proof. Removing a circle from an admissible (or coadmissible) circle diagram clearly produces an admissible (or coadmissible) circle diagram. Thus $\mathcal{Q}$ is admissible. Let $A_{\mathcal{Q}}, B_{\mathcal{Q}}, C_{\mathcal{Q}}, D_{\mathcal{Q}}$ be the associated subsets of $\mathcal{Q}$. We consider the case where $\mathcal{Q}$ is formed by inserting a puncture at the $j$ th position of $F\left(A_{\mathcal{P}}-\{a\}, D_{\mathcal{Q}}\right)$ and $(l, r)_{j}=(\overline{\mathbf{a}}, \overline{\mathbf{c}})$. Here we have $a \in A_{\mathcal{P}}$ and $c \in B_{\mathcal{P}}$. By the construction of $\mathcal{Q}$, $A_{\mathcal{Q}}=A_{\mathcal{P}}-\{a\}$ and $D_{\mathcal{Q}}=D_{\mathcal{P}}$. To show $l \mathcal{P} \leq l \mathcal{Q}$ and $r \mathcal{Q} \leq r \mathcal{P}$ it is sufficient to show $C_{\mathcal{P}} \leq C_{\mathcal{Q}}$ and $B_{\mathcal{Q}} \geq B_{\mathcal{P}}-\{c\}$. Lemma 8.3(1) implies that $B_{\mathcal{Q}}=B_{\mathcal{P}}-\{b\}$ and $C_{\mathcal{Q}}=\left(C_{\mathcal{P}}+\{a\}\right)-\{b\}$, where $b:=\max \left[\{1,2, \ldots, a\} \backslash U_{\mathcal{Q}}\right]$. Since $b \leq a$ we have $C_{\mathcal{P}} \leq C_{\mathcal{Q}}$. Also by Lemma 8.3, we have $j=\operatorname{index}[\overline{\mathbf{a}}, l \mathcal{P}] \leq$ index $[\overline{\mathbf{b}}, r \mathcal{P}]$. Since $j=$ index $[\overline{\mathbf{c}}, r \mathcal{P}]$, it follows that $\overline{\mathbf{c}} \leq \overline{\mathbf{b}}$ and so $c \geq b$. Thus $B_{\mathcal{Q}}=B_{\mathcal{P}}-\{b\} \geq B_{\mathcal{P}}-\{c\}$. To see the other cases, apply the symmetries of $K_{4}$ to this argument.

\section{Proofs FOR THE SJDT-ALGORITHM}

The various parts of Theorem 7.3 are proven in this section. In the following lemma we establish the well definedness of the horizontal move. Fix $n \geq 1$. Let $\mathcal{T}$ be a punctured admissible skew tableau whose entries are from $[[\mathbf{n}]]$. Suppose the puncture is at $(p, q)$ and suppose that either $(p+1, q)$ is empty or $r_{p+1, q}>l_{p, q+1}$. Let $\mathcal{T}^{\prime}=\mathbf{h}_{R}(\mathcal{T},(p, q))$. Let $\mathcal{T}_{i}$ denote the $i$ th column of $\mathcal{T}$. Attach $\left(^{\prime}\right)$ to denote any quantity associated to $\mathcal{T}^{\prime}$.

Lemma 9.1. (1) $\mathcal{T}^{\prime}$ is a punctured skew tableaux with puncture at $(p, q+1)$, with admissible columns, and such that either $(p-1, q+1)$ is empty or $l_{p-1, q+1}^{\prime}<r_{p, q}^{\prime}$. (2) $\operatorname{wt}\left(\mathcal{T}^{\prime}\right)=\operatorname{wt}(\mathcal{T})$. (3) The entries of $\mathcal{T}^{\prime}$ are from $[[\mathbf{n}+\mathbf{1}]]$. If an $\mathbf{n}+\mathbf{1}$ or $\overline{\mathbf{n}+\mathbf{1}}$ appears in $\mathcal{T}^{\prime}$, then each of these two symbols appears exactly once and each in the qth column of $\mathcal{T}^{\prime}$. The entry $l_{p, q+1}$ of $\mathcal{T}$ was barred. (4) Most importantly, $r \mathcal{T}_{q}^{\prime} \leq l \mathcal{T}_{q+1}$.

Proof. Let $A_{i}, B_{i}, C_{i}, D_{i}$ denote its associated subsets of $\mathcal{T}_{i}$. We start with part (1). It is evident that $\mathcal{T}^{\prime}$ is a punctured skew tableaux with puncture at $(p, q+1)$. Assume $l_{p, q+1}$ is a barred symbol. Then the barred entry region of the adjacent split columns $r \mathcal{T}_{q}$ and $l \mathcal{T}_{q+1}$ are the same as the barred entries of $r \mathcal{T}_{n}^{\prime}$ and $l \mathcal{T}_{q+1}^{\prime}$, except the puncture at $r_{p, q}$ and the entry $l_{p, q+1}$ are interchanged. If $(p-1, q+1)$ is not empty then $l_{p-1, q+1}^{\prime}=l_{p-1, q+1}<l_{p, q+1}=r_{p, q}^{\prime}$. The argument is similar when $l_{p, q+1}$ is unbarred. (See the remarks following the example of the horizontal move in Section 7.)

Set $c=\left\{l_{p, q+1}\right\}$. We verify the admissibility of all the columns possibly constructed by Subroutine 7.1: $F\left(A_{q+1}-\{c\}, D_{q+1}\right), G\left(B_{q+1}, C_{q+1}-\{c\}\right), F\left(A_{q}, D_{q}+\right.$ $\{c\})$ and $G\left(B_{q}+\{c\}, C_{q}\right)$. It is obvious that the column $F\left(A_{q+1}-\{c\}, D_{q+1}\right)$ is admissible. By Lemma 5.1 the columns $G\left(B_{q+1}, C_{q+1}-\{c\}\right)$ and $G\left(B_{q}+\{c\}, C_{q}\right)$ are admissible. This leaves $F\left(A_{q}, D_{q}+\{c\}\right)$. Here $l_{p, q+1}=\mathbf{c}$. Let $h_{q}$ and $h_{q+1}$ be the number of leading empty boxes in $\mathcal{T}_{q}$ and $\mathcal{T}_{q+1}$ respectively. Consider the column 
$l \mathcal{T}_{q+1}=F\left(A_{q+1}, C_{q+1}\right)$. It is itself an admissible column since $A_{q+1} \cap C_{q+1}=\varnothing$. Lemma 8.6 implies $c \geq p-\left(h_{q+1}+\left|\left\{x \in A_{q+1}: x>c\right\}\right|\right)$. By assumption we have $\mathcal{T}_{q} \leq r \mathcal{T}_{q} \leq l \mathcal{T}_{q+1}$. This implies that $h_{q}+\left|\left\{x \in A_{q}: x>c\right\}\right| \geq h_{q+1}+\mid\left\{x \in A_{q+1}:\right.$ $x>c\} \mid$. Thus $c \geq p-\left(h_{q}+\left|\left\{x \in A_{q}: x>c\right\}\right|\right)$, and the inequality of Lemma 8.6 is satisfied for the new unbarred $\mathbf{c}$ in $F\left(A_{q}, D_{q}+\{c\}\right)$. Hence the column is admissible. This completes part (1).

Note that $\mathbf{h}_{R}$ changes only the $q$ th and $(q+1)$ st columns. Consider the case $l_{p, q+1}=\mathbf{c}$. Replacing $\mathcal{T}_{q}$ with $F\left(A_{q}, D_{q}+\{c\}\right)$ increase the $c$ th component of wt $(\mathcal{T})$ by one. Replacing $\mathcal{T}_{q+1}$ with $G\left(B_{q+1}, C_{q+1}-\{c\}\right)$ decreases the $c$ th component of $\operatorname{wt}(\mathcal{T})$ by one. Since the actions cancel each other, $\operatorname{wt}\left(\mathcal{T}^{\prime}\right)=\operatorname{wt}(\mathcal{T})$. A similar argument applies to the case $l_{p, q+1}=\overline{\mathbf{c}}$.

Now for part (3). The entries of $F\left(A_{q+1}-\{c\}, D_{q+1}\right), G\left(B_{q+1}, C_{q+1}-\{c\}\right)$, and $F\left(A_{q}, D_{q}+\{c\}\right)$ stay in [[n]]. So if $\mathbf{n}+\mathbf{1}$ or $\overline{\mathbf{n}+\mathbf{1}}$ appears in $\mathcal{T}^{\prime}$, the definition of $\mathbf{h}_{R}$ implies that $l_{p, q+1}$ is a barred entry. Assume $l_{p, q+1}=\overline{\mathbf{c}}$. We show the entries of $G\left(B_{q}+\{c\}, C_{q}\right)$ are in $[[\mathbf{n}+\mathbf{1}]]$. Recall that $G\left(B_{q}, C_{q}\right)$ is the admissible $2 \times n$ circle diagram obtained by first placing circles corresponding to $B_{q}$ and $C_{q}$, and then shifting the circles in the full slots to the leftmost empty slots to their right. Adding a circle to $B_{q}$ either creates one new full slot or destroys one empty slot. Add an empty $(n+1)$ st slot to $G\left(B_{q}, C_{q}\right)$. No empty slots to the right of this slot will be needed to form $G\left(B_{q}+\{c\}, C_{q}\right)$. If this slot is used, both an $\mathbf{n}+\mathbf{1}$ and an $\overline{\mathbf{n}+\mathbf{1}}$ will appear in $\mathcal{T}_{q}^{\prime}$. Thus $G\left(B_{q}+\{c\}, C_{q}\right)$ corresponds to a $2 \times(n+1)$ admissible circle diagram.

For part (4) we show that $r \mathcal{T}_{q}^{\prime} \leq l \mathcal{T}_{q+1}$. First suppose $l_{p, q+1}=\overline{\mathbf{c}}$ is barred. To form the barred region of $r \mathcal{T}_{q}^{\prime}$ and $l \mathcal{T}_{q+1}^{\prime}$ from the barred region of $r \mathcal{T}_{q}$ and $l \mathcal{T}_{q+1}^{\prime}$, the subroutine $\mathbf{h}_{R}$ simply interchanges the puncture with $\overline{\mathbf{c}}$. So focus on the unbarred region of these columns. Set $U_{q}=A_{q} \cup B_{q} \cup C_{q} \cup D_{q}$ and $d=$ $\min \left[\{n+1, n, n-1, \ldots, c\} \backslash U_{q}\right]$. Since part (1) tells us that $\mathcal{T}_{q}^{\prime}$ is admissible, Lemma 8.3(2) tells us that $D_{q}=\left(D_{q}^{\prime}+\{c\}\right)-\{d\}$. If it were the case that $c=d$ then $D_{q}^{\prime}=D_{q}$ and the claim $r \mathcal{T}_{q}^{\prime} \leq l \mathcal{T}_{q+1}$ follows easily. So assume that $d>c$. Since $D_{q}=\left(D_{q}^{\prime}+\{c\}\right)-\{d\}$, we have $c \in D_{q}$. Let $t$ be such that $r_{t, q}=\mathbf{c}$, and let $v$ be maximal such that $r_{t+v, q}<\mathbf{d}$. The column $r \mathcal{T}_{q}^{\prime}=F\left(B_{q}+\{c\},\left(D_{q}-\{c\}\right)+\{d\}\right)$ is formed from $r \mathcal{T}_{q}$ as follows: fill the puncture with the $\overline{\mathbf{c}}$; remove the $\mathbf{c}$ from $r \mathcal{T}_{q}$ creating a space at $r_{t, q}$; slide the entries $r_{t+1, q}, r_{t+2, q}, \ldots, r_{t+v, q}$ up one box so that the $(t+v)$ th box is empty; insert the $\mathbf{d}$ there. To show $r \mathcal{T}_{q}^{\prime} \leq l \mathcal{T}_{q+1}$ it is sufficient to show $r_{t+1, q} \leq l_{t, q+1}, r_{t+2, q} \leq l_{t+1, q+1}, \ldots, r_{t+v, q} \leq l_{t+v-1, q+1}$ and $\mathbf{d} \leq l_{t+v, q+1}$. (If the length of $l \mathcal{T}_{q+1}$ is less than $t+v$, add entries $\mathbf{n}+\mathbf{1}, \mathbf{n}+\mathbf{2}, \ldots$ to the end of the column until it attains this length.) Let $u$ be maximal such that $r_{p-u, q}>\overline{\mathbf{d}}$ (i.e. $\left\|r_{p-u, q}\right\|<d$ ) or set $u=0$ if $r_{p-1, q}<\overline{\mathbf{d}}$. Set

$$
\begin{aligned}
\widetilde{B}_{q} & =\left\{\left\|r_{p-u, q}\right\|>\left\|r_{p-u+1, q}\right\|>\cdots>\left\|r_{p-1, q}\right\|\right\}, \\
\widetilde{A}_{q+1} & =\left\{\left\|l_{p-u, q+1}\right\|>\left\|l_{p-u+1, q+1}\right\|>\cdots>\left\|l_{p-1, q+1}\right\|\right\}, \\
\widetilde{D}_{q} & =\left\{\left\|r_{t+1, q}\right\|<\left\|r_{t+2, q}\right\|<\cdots<\left\|r_{t+v, q}\right\|<d\right\}, \\
\widetilde{C}_{q+1} & =\left\{\left\|l_{t, q+1}\right\|<\left\|l_{t+1, q+1}\right\|<\cdots<\left\|l_{t+v, q+1}\right\|\right\}
\end{aligned}
$$

(where $\widetilde{B}_{q}=\widetilde{A}_{q+1}=\varnothing$ if $u=0$ ). Now we must show that $\widetilde{D}_{q} \leq \widetilde{C}_{q+1}$. We have $\left|\widetilde{D}_{q}\right|=\left|\widetilde{C}_{q+1}\right|=v+1$. Note that the partially ordered set $\{x: X \subseteq[c+$ $\left.1, \infty)-\widetilde{B}_{q},|X|=v+1\right\}$ has a unique minimal element. This set contains $\widetilde{D}_{q}$ since 
$\left\|r_{t+1, q}\right\| \geq c+1$ and $\widetilde{D}_{q} \cap \widetilde{B}_{q}=\varnothing$. Since $d=\min \left[\{n+1, n, n-1, \ldots, c\} \backslash U_{q}\right]$ and $U_{q}=B_{q}+D_{q}$, we have $\widetilde{D}_{q}+\widetilde{B}_{q}=[c+d]$. Thus, in fact $\widetilde{D}_{q}=\min [X$ : $\left.X \subseteq[c+1, \infty)-\widetilde{B}_{q},|X|=v+1\right]$. Since $\left\|l_{p-1, q+1}\right\|>\left\|l_{p, q+1}\right\|=c$ we have $\widetilde{A}_{q+1} \subseteq[c+1, \infty)$. Also, we have $\left\|l_{t, q+1}\right\| \geq\left\|r_{t, q}\right\|=c$. So $\widetilde{C}_{q+1} \subseteq[c, \infty)$. But since $c \in A_{q+1}$ and $A_{q+1} \cap C_{q+1}=\varnothing$ we must have $\widetilde{C}_{q+1} \subseteq[c+1, \infty)$. Thus $\widetilde{C}_{q+1}$ is an element of $\left\{X: X \subseteq[c+1, \infty)-\widetilde{A}_{q+1},|X|=v+1\right\}$. This latter set also has a unique minimal element. Thus $\widetilde{C}_{q+1} \geq \min \left[X: X \subseteq[c+1, \infty)-\widetilde{A}_{q+1},|X|=v+1\right]$. Our assumption $r \mathcal{T}_{q} \leq l \mathcal{T}_{q+1}$ implies $\widetilde{B}_{q} \geq \widetilde{A}_{q+1}$. It follows that $\widetilde{D}_{q} \leq \min [X$ : $\left.X \subseteq[c+1, \infty)-\widetilde{A}_{q+1},|X|=v+1\right] \leq \widetilde{C}_{q+1}$.

The argument for unbarred $l_{p, q+1}$ is similar and has been dropped from the journal version of this paper.

Lemma 9.2. (1) The sjdt-algorithm is well defined and provides a map:

$$
\text { sjdt : } \bigcup_{\lambda \backslash \mu} \mathcal{D}(\lambda \backslash \mu) \times\{\text { inner corners of } \mu\} \rightarrow \bigcup_{\lambda \backslash \mu} \mathcal{D}(\lambda \backslash \mu) \times\{\text { outer corners of } \lambda\} \text {. }
$$

(2) Fix $n \geq 1$ and let $(\mathcal{T},(x, y)) \in \mathcal{D}(\lambda \backslash \mu, n) \times\{$ inner corners of $\mu\}$. Set $(\mathcal{S},(q, z)):=\operatorname{sjdt}(\mathcal{T},(x, y))$. If an $\mathbf{n}+\mathbf{1}$ or $\overline{\mathbf{n}+\mathbf{1}}$ appears in $\mathcal{S}$, then each of these two symbols appear exactly once in the yth column and nowhere else.

Proof. To show well-definedness we verify that after each move of the puncture, the split-column form of the resulting punctured tableau is admissible. It is clear that the vertical moves preserve admissibility so we need only consider the tableaux resulting from a horizontal move. The proof is by induction on the number $k$ of horizontal moves performed during the algorithm. We use the following notation. If $k>1$ let $\mathcal{T}_{j}^{-}$denote the $j$ th column of $\mathcal{T}$ just before the $(k-1)$ st horizontal move is made (just before the puncture leaves the, say, $(q-1)$ st column). Denote by $\mathcal{T}_{j}^{+}$ the $j$ th-column just after the $k$ th horizontal move is made (just after the puncture enters the $(q+1)$ st-column). We use $\mathcal{T}_{j}$, with no superscript, to refer to the $j$ th column at the various steps between these two moves, when the puncture is in the $q$ th column. Superscript the associated subsets accordingly. If $k=1$ let $\mathcal{T}_{j}^{-}$be the $j$ th column of the input tableau and set $A_{q}^{-}=A_{q}+\{n+1\}$ and $B_{q}^{-}=B_{q}+\{n+1\}$.

Suppose that $\mathcal{T}$ remained admissible after the first $k-1$ horizontal moves were made, the puncture is at $(p, q)$, and we are about to perform the $k$ th horizontal move. Lemma 9.1(1) implies that each individual column of $\mathcal{T}$ is admissible after the $k$ th horizontal move. Since the $k$ th horizontal move changes only the $q$ th and $(q+1)$ st columns, it is sufficient to show: (I) $r \mathcal{T}_{q-1}^{+} \leq l \mathcal{T}_{q}^{+}$, (II) $r \mathcal{T}_{q}^{+} \leq l \mathcal{T}_{q+1}^{+}$, and (III) $r \mathcal{T}_{q+1}^{+} \leq l \mathcal{T}_{q+2}^{+}$. Lemma 8.6 implies that for the punctured column $\mathcal{T}_{q+1}^{+}$we have $l \mathcal{T}_{q+1} \leq l \mathcal{T}_{q+1}^{+}$and $r \mathcal{T}_{q+1}^{+} \leq r \mathcal{T}_{q+1}$. Since $r \mathcal{T}_{q+1} \leq l \mathcal{T}_{q+2}=l \mathcal{T}_{q+2}^{+}$, we have (III). Lemma 9.1(4) states that $r \mathcal{T}_{q}^{+} \leq l \mathcal{T}_{q+1}$, so we can deduce (II). When $k>1$ the proof of (I) splits into three cases depending upon where the two horizontal moves involved in the puncture's pass through the $q$ th column take place: (i) both in the barred entry region, (ii) the first in the barred region and the second in the unbarred region, or (iii) both in the unbarred entry region. When $k=1$ the proof of (I) is handled in Case (i) if the first horizontal move occurs in the barred region and in Case (ii) if it occurs in the unbarred region. For (I) it is sufficient to show that in each case $l \mathcal{T}_{q}^{-} \leq l \mathcal{T}_{q}^{+}$: Lemma 9.1(4) states that $r \mathcal{T}_{q-1} \leq l \mathcal{T}_{q}^{-}$. 
Since $r \mathcal{T}_{q-1}=r \mathcal{T}_{q-1}^{+}$we then have (I). It is helpful to refer to the figure below; the correspondence with the text is given by $\|\boldsymbol{x}\|=a,\|\boldsymbol{y}\|=b,\|\boldsymbol{z}\|=c$, and $\|\boldsymbol{w}\|=d$.
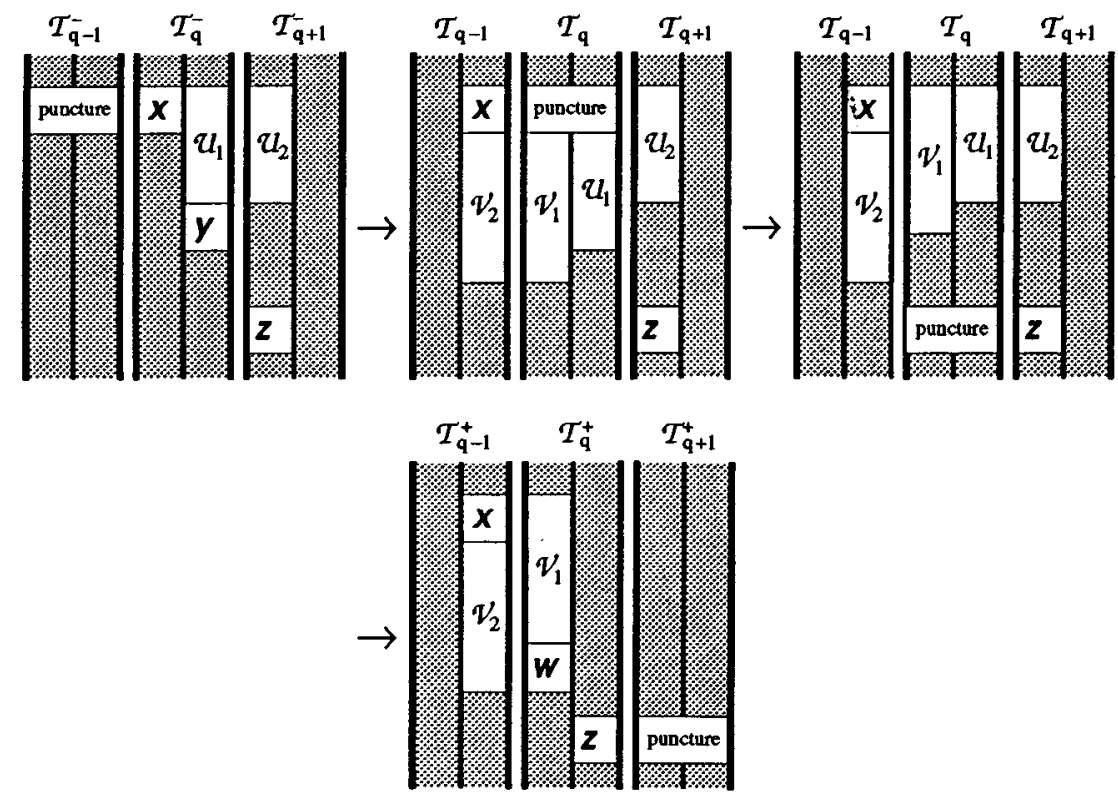

Case (i): Assume the puncture enters column $q$ in its barred entry region and also enters column $q+1$ in its barred entry region. In the figure, $\boldsymbol{x}$ and $\boldsymbol{z}$ are both barred symbols. Let $(p, q)$ be the location of the puncture in $\mathcal{T}_{q}$ just after the $(k-1)$ st horizontal move. For now assume $k>1$. Let $a=\|\boldsymbol{x}\|$ so that $A_{q}=A_{q}^{-}-$ $\{a\}, D_{q}=D_{q}^{-}$. Apply Lemma $8.3(1)$ so that we have $b=\max \left[\{1,2, \ldots, a\} \backslash U_{q}\right]$, $B_{q}=B_{q}^{-}-\{b\}, C_{q}=\left(C_{q}^{-}+\{a\}\right)-\{b\}$. Note that $p=\operatorname{index}\left[\overline{\mathbf{a}}, l \mathcal{T}_{q}^{-}\right]$. Let $s=\operatorname{index}\left[\overline{\mathbf{b}}, r \mathcal{T}_{q}^{-}\right]$. By Lemma $8.3(1), p \leq s$. If $p<s$ then $r \mathcal{T}_{q}$ is formed from $r \mathcal{T}_{q}^{-}$ as follows: remove the $\overline{\mathbf{b}}$ from its box and slide the $p$ th through $s-1$ st entries of $r \mathcal{T}_{q}^{-}$down. (See the first two frames in the figure above where the entries $p$ through $s-1$ form the subcolumn $\mathcal{U}_{1}$ of $r \mathcal{T}_{q}^{-}$.) Since we are assuming $r \mathcal{T}_{q}^{-} \leq l \mathcal{T}_{q+1}^{-}=l \mathcal{T}_{q+1}$, at least $s-p$ vertical moves are required between the $k-1$ st and $k$ th horizontal moves. Thus $c \leq b$, where $\overline{\mathbf{c}}=\mathbf{z}$ is the element of $l \mathcal{T}_{q+1}$ to the right of the puncture just before the $k$ th horizontal move. If $p=s$ then $c \leq b$ is obvious. From now on let $k \geq 1$. If $k=1$, set $a=b=n+1$; then $c \leq b$ is obvious. For $k \geq 1$ note that $B_{q}=B_{q}^{+}-\{c\}$ and $C_{q}=C_{q}^{-}$. Apply Lemma 8.3(2) to get $d=\min \left[\{n+1, n, n-1, \ldots, c\} \backslash U_{q}\right], A_{q}=A_{q}^{+}-\{d\}$ and $D_{q}=\left(D_{q}^{+}+\{c\}\right)-\{d\}$. To show $l \mathcal{T}_{q}^{-} \leq l \mathcal{T}_{q}^{+}$we need $A_{q}^{-} \geq A_{q}^{+}$and $C_{q}^{-} \leq C_{q}^{+}$. Note that $c \leq b \leq a$ and $b$ is not an element of $U_{q}$. Thus $d \leq b$, and so $d \leq a$. Since $A_{q}^{+}=\left(A_{1}^{-}-\{a\}\right)+\{d\}$ we have $A_{q}^{-} \geq A_{q}^{+}$. Also, $C_{q}^{+}=\left(C_{q}^{-}+\{a\}\right)-\{b\}$ implies $C_{q}^{-} \leq C_{q}^{+}$.

Now for the proof of part (2): By Lemma $9.1(3)$ the symbols $\mathbf{n}+\mathbf{1}$ and $\overline{\mathbf{n}+\mathbf{1}}$ can arise only in the column on the left after the puncture slides out of it. This would be $\mathcal{T}_{q}^{+}$. But the new entries in $\mathcal{T}_{q}^{+}$are $\mathbf{d}$ and $\overline{\mathbf{d}}$, and from above we have $d \leq b \leq a$. Since $b \leq a \leq n$ when $k>1$, an $\mathbf{n}+\mathbf{1}$ and $\overline{\mathbf{n + 1}}$ can arise only when $k=1$. Clearly $q=y$. 
Case (ii): Assume the puncture enters column $q$ in its barred entry region and enters column $q+1$ in its unbarred entry region. Now the $\boldsymbol{x}$ is barred and $\boldsymbol{z}$ is unbarred in the figure above. Let $(p, q), a$ and $b$ be as they were in case (i). (Set $a=b=n+1$ if $k=1$.) Again $A_{q}=A_{q}^{-}-\{a\}$. Let $c=\|\boldsymbol{z}\|$. Then $D_{q}=D_{q}^{-}-\{c\}$ and $A_{q}=A_{q}^{+}$. Lemma 8.3(4) implies $C_{q}=C_{q}^{+}-\{d\}$ where $d:=\max \left[\{1,2, \ldots, c\} \backslash U_{q}\right]$. We need to show that $l \mathcal{T}_{q}^{-} \leq l \mathcal{T}_{q}^{+}$. By Lemma 8.7 we have $l \mathcal{T}_{q}^{-} \leq l \mathcal{T}_{q}$, where this $l \mathcal{T}_{q}$ is at the step just after the $(k-1)$ st horizontal move. Form the semistandard column $\mathcal{P}=F\left(A_{q}^{-}, C_{q}\right)$ from the barred entries of $l \mathcal{T}_{q}^{-}$and the unbarred entries of $l \mathcal{T}_{q}$. If column $q$ has any leading empty boxes, insert these at the top of $\mathcal{P}$. Then $l \mathcal{T}_{q}^{-} \leq \mathcal{P}$. Note that $A_{q}^{+}=A_{q}^{-}-\{a\}$ and $C_{q}^{+}=C_{q}+\{d\}$. We have $l \mathcal{T}_{q}^{+}=F\left(A_{q}^{-}-\{a\}, C_{q}+\{d\}\right)=(\mathcal{P}-\{\overline{\mathbf{a}}\})+\{d\}$ where the columns are viewed as subsets of $[[\mathbf{n}]]$. Thus $\mathcal{P} \leq l \mathcal{T}_{q}^{+}$. This implies $l \mathcal{T}_{q}^{-} \leq l \mathcal{T}_{q}^{+}$.

Case (iii): Assume the puncture enters and leaves column $q$ in its unbarred entry region. Here both $\boldsymbol{x}$ and $\boldsymbol{z}$ in the figure are unbarred and $k>1$. Let $(p, q)$ be the location of the puncture in $\mathcal{T}_{q}$ just after the $(k-1)$ st horizontal move. Reassign $a$ so that $C_{q}=C_{q}^{-}-\{a\}, B_{q}=B_{q}^{-}$, and use Lemma 8.3(3) to reassign $b$ as $b:=\min \left[\{n, n-1, \ldots, a\} \backslash U_{q}\right], D_{q}=D_{q}^{-}-\{b\}, A_{q}=\left(A_{q}^{-}+\{a\}\right)-\{b\}$. Let $c$ and $d$ be as they were in case (ii): $D_{q}=D_{q}^{+}-\{c\}, A_{q}=A_{q}^{+}, C_{q}=C_{q}^{+}-\{d\}$, $B_{q}=\left(B_{q}^{+}+\{c\}\right)-\{d\}$, where $d:=\max \left[\{1,2, \ldots, c\} \backslash U_{q}\right]$. Since $b \geq a$ and $A_{q}^{+}=\left(A_{q}^{-}+\{a\}\right)-\{b\}$, we have $A_{q}^{-} \geq A_{q}^{+}$. The same shifting argument given in case (i) to show $c \leq b$ shows in this case $b \leq c$. Since $b \notin U_{q}$ we must have $b \leq d$, and so $a \leq d$. Since $C_{q}^{+}=\left(C_{q}^{-}-\{a\}\right)+\{d\}$ we have $C_{q}^{+} \geq C_{q}^{-}$. Thus $l \mathcal{T}_{q}^{-} \leq l \mathcal{T}_{q}^{+}$.

Part (1) of Theorem 7.3 is evident from the definition of the algorithm and from 9.1(2). Part (3) and the well-definedness claim of Theorem 7.3 were proved in Proposition 9.2. This leaves parts (2) and (4) and the bijection claim. We begin with a continuation of the proof of Proposition 9.2. This leads to half of Theorem 7.3(2). When the puncture passed through a split-column $\left(l \mathcal{T}_{q}, r \mathcal{T}_{q}\right)$ four elements $\boldsymbol{x}, \boldsymbol{y}, \boldsymbol{z}, \boldsymbol{w} \in[[\mathbb{P}]]$ were involved. The puncture enters column $\mathcal{T}_{q}$ displacing the entry $\boldsymbol{x}$ of $l \mathcal{T}_{q}^{-}$; the entry $\boldsymbol{y}$ of $r \mathcal{T}_{q}^{-}$is eliminated. In the notation of Lemmas 8.4 and 8.5 we have $\boldsymbol{y}=L_{\mathcal{T}_{q}^{-}}(\boldsymbol{x})$. The puncture moves down the $q$ th column and then exits, being replaced by the entries $\boldsymbol{z}$ and $\boldsymbol{w}$ : the entry $\boldsymbol{z}$ is moved into $r \mathcal{T}_{q}^{+}$and the entry $\boldsymbol{w}$ appears in $l \mathcal{T}_{q}^{+}$through the application of $\mathbf{h}_{R}$. This implies $\boldsymbol{w}=R_{\mathcal{T}_{q}^{+}}(\boldsymbol{z})$. Set $i_{\boldsymbol{x}}=\operatorname{index}\left[\boldsymbol{x}, l \mathcal{T}_{q}^{-}\right], i_{\boldsymbol{y}}=\operatorname{index}\left[\boldsymbol{y}, r \mathcal{T}_{q}^{-}\right], i_{\boldsymbol{z}}=\operatorname{index}\left[\boldsymbol{z}, r \mathcal{T}_{q}^{+}\right]$, and $i_{\boldsymbol{w}}=\operatorname{index}\left[\boldsymbol{w}, l \mathcal{T}_{q}^{+}\right]$.

Lemma 9.3. In the proof of Proposition $9.2, i_{\boldsymbol{x}} \leq i_{\boldsymbol{y}} \leq i_{\boldsymbol{w}} \leq i_{\boldsymbol{z}}$.

Proof. Lemma 8.3 shows that $i_{\boldsymbol{x}} \leq i_{\boldsymbol{y}}$ and $i_{\boldsymbol{w}} \leq i_{\boldsymbol{z}}$. We refer to the proof of Proposition 9.2 to show that $i_{\boldsymbol{w}} \leq i_{\boldsymbol{y}}$ : We have $a=\|\boldsymbol{x}\|, b=\|\boldsymbol{y}\|, c=\|\boldsymbol{z}\|$, and $d=\|\boldsymbol{w}\|$. We argue only Case (i) of that proof where $\boldsymbol{x}, \boldsymbol{y}, \boldsymbol{z}, \boldsymbol{w}$ are all barred symbols. Case (ii) is easier since $\boldsymbol{y}$ is barred and $\boldsymbol{w}$ is unbarred. The argument for Case (iii), where $\boldsymbol{x}, \boldsymbol{y}, \boldsymbol{z}, \boldsymbol{w}$ are all unbarred, is similar to Case (i). For Case (i) we have $l \mathcal{T}_{q}^{-}=F\left(A_{q}^{-}, C_{q}^{-}\right), r \mathcal{T}_{q}^{-}=F\left(B_{q}^{-}, D_{q}^{-}\right)$and $l \mathcal{T}_{q}^{+}=F\left(\left(A_{q}^{-}-\{a\}\right)+\{d\},\left(C_{q}^{-}+\right.\right.$ $\{a\})-\{b\}), r \mathcal{T}_{q}^{+}=F\left(\left(B_{q}^{-}-\{b\}\right)+\{c\},\left(D_{q}^{-}+\{d\}\right)-\{c\}\right)$. So $i_{\boldsymbol{y}}=\left|\left\{u \in B_{q}^{-}: u \geq b\right\}\right|$ and $i_{\boldsymbol{w}}=\left|\left\{u \in\left(A_{q}^{-}-\{a\}\right)+\{d\}: u \geq d\right\}\right|$. For Case (i), $d \leq b \leq a$. Thus we have $i_{\boldsymbol{w}}=\left|\left\{u \in A_{q}^{-}: u \geq d\right\}\right| \geq\left|\left\{u \in A_{q}^{-}: u \geq b\right\}\right|$. Since $A_{q}^{-} \geq B_{q}^{-}$, we have $i_{\boldsymbol{w}} \geq\left|\left\{u \in A_{q}^{-}: u \geq b\right\}\right| \geq\left|\left\{u \in B_{q}^{-}: u \geq b\right\}\right|=i_{\boldsymbol{y}}$. 
Now we consider applying the sjdt-algorithm twice. The following lemma is the key step in the inductive proof of Theorem 7.3(2).

Lemma 9.4. Let $\mathcal{T}$ be an admissible skew tableau. Use the sjdt-algorithm on $\mathcal{T}$ to slide a first puncture all the way out. Then use the sjdt-algorithm on the resulting tableau to slide a second puncture all the way out. Suppose both punctures pass through the qth column: the first puncture entering at row $i_{\boldsymbol{x}}$ and exiting at row $i_{\boldsymbol{z}}$; the second entering at row $i_{\boldsymbol{x}^{\prime}}$ and exiting at row $i_{\boldsymbol{z}^{\prime}}$. If $i_{\boldsymbol{x}^{\prime}} \geq i_{\boldsymbol{x}}$ then $i_{\boldsymbol{z}^{\prime}} \geq i_{\boldsymbol{z}}$.

Proof. Let $\boldsymbol{x}, \boldsymbol{y}, \boldsymbol{z}, \boldsymbol{w}$ be related to the first puncture's pass through column $q$ as in the discussion prior to Lemma 9.3. At the step after the first puncture enters the $q$ th split-column, let $\mathcal{V}_{1}$ be the subcolumn of $l \mathcal{T}_{q}$ consisting of all entries strictly between $\boldsymbol{x}$ and $\boldsymbol{w}\left(\mathcal{V}_{1}\right.$ may be empty). Let $\mathcal{V}_{2}$ be the subcolumn of $r \mathcal{T}_{q-1}$ directly to the left of $\mathcal{V}_{1}$ (see the second frame in the figure in the proof of Proposition 9.2). As the puncture moves to row $i_{\boldsymbol{z}}$, the subcolumn $\mathcal{V}_{1}$ is slid up one box. The subsequent horizontal move inserts the $\boldsymbol{w}$ directly under the subcolumn $\mathcal{V}_{1}$ (or at $i_{\boldsymbol{x}}$ if $\mathcal{V}_{1}$ was empty). Now assume the puncture is slid out to the perimeter of the tableau and the sjdt-algorithm is applied a second time. At the point immediately after the second puncture enters column $q-1$, let $\widetilde{\mathcal{V}}_{2}$ be the same portion of that column as was occupied by $\mathcal{V}_{2}$. By Lemma 8.7 we have $\widetilde{\mathcal{V}}_{2} \leq \mathcal{V}_{2}$. From this and our assumption $i_{\boldsymbol{x}^{\prime}} \geq i_{\boldsymbol{x}}$ we know that the second puncture will reach at least the $i_{\boldsymbol{w}}$ th row of column $q-1$. Denote by $\boldsymbol{x}^{\prime}, \boldsymbol{y}^{\prime}$ the two elements of $[[\mathbb{P}]]$ associated as above to the second puncture's entry into column $q$ with $i_{\boldsymbol{x}^{\prime}}=\operatorname{index}\left[\boldsymbol{x}^{\prime}, l \mathcal{T}_{q}^{+}\right]$ and $i_{\boldsymbol{y}^{\prime}}=\operatorname{index}\left[\boldsymbol{y}^{\prime}, r \mathcal{T}_{q}^{+}\right]$. Note that $\boldsymbol{y}^{\prime}=L_{\mathcal{T}_{q}^{+}}\left(\boldsymbol{x}^{\prime}\right)$. Recall from the paragraph preceding Lemma 9.3 that $\boldsymbol{w}=R_{\mathcal{T}_{q}^{+}}(\boldsymbol{z})$. Since the second puncture reaches at least the $i_{\boldsymbol{w}}$ th row of column $q-1, \boldsymbol{x}^{\prime} \geq \boldsymbol{w}$. Lemma 8.5 then implies

$$
\boldsymbol{y}^{\prime}=L_{\mathcal{T}_{q}^{+}}\left(\boldsymbol{x}^{\prime}\right) \geq L_{\mathcal{T}_{q}^{+}}(\boldsymbol{w})=L_{\mathcal{T}_{q}^{+}}\left(R_{\mathcal{T}_{q}^{+}}(\boldsymbol{z})\right) \geq \boldsymbol{z} .
$$

Since $\boldsymbol{z}$ and $\boldsymbol{y}^{\prime}$ are both symbols in $r \mathcal{T}_{q}^{+}, \boldsymbol{z} \leq \boldsymbol{y}^{\prime}$ iff $i_{\boldsymbol{z}} \leq i_{\boldsymbol{y}^{\prime}}$. If the second puncture leaves column $q$ at row $i_{\boldsymbol{z}^{\prime}}$, then by Lemma $9.3 i_{\boldsymbol{y}^{\prime}} \leq i_{\boldsymbol{z}^{\prime}}$. Thus $i_{\boldsymbol{z}} \leq i_{\boldsymbol{z}^{\prime}}$.

For later use we record the result $(*)$ above in the next lemma. The hypothesis $\boldsymbol{x}^{\prime} \geq \boldsymbol{x}$ here is easily seen to be equivalent to the hypothesis $i_{\boldsymbol{x}^{\prime}} \geq i_{\boldsymbol{x}}$ above.

Lemma 9.5. Suppose the sjdt-algorithm is applied twice to a skew tableau $\mathcal{T}, \mathcal{T} \rightarrow$ $\operatorname{sjdt}\left(\operatorname{sjdt}(\mathcal{T},(u, v))_{1},\left(u^{\prime}, v^{\prime}\right)\right)_{1}$. Let $\boldsymbol{x}, \boldsymbol{z} \in[[\mathbb{P}]]$ be associated (as above) to the first puncture's pass through the qth column. Suppose the second puncture reaches column $q$ and let $\boldsymbol{x}^{\prime}, \boldsymbol{y}^{\prime} \in[[\mathbb{P}]]$ be associated to this puncture's entry therein. If $\boldsymbol{x}^{\prime} \geq \boldsymbol{x}$ then $\boldsymbol{y}^{\prime} \geq \boldsymbol{z}$.

Now we prove half of Theorem $7.3(2)$.

Proof of part of Theorem 7.3(2). We prove that if $y^{\prime}<y$ then $z^{\prime}<z$, and the paths the two puncture take as the sjdt-algorithm is applied never cross. We induct on the index $q$ of the columns the second puncture enters during the application of the sjdt-algorithm. To start the induction note that if $y^{\prime}<y$, then because $\mu^{\prime}=\mu-\{(x, y)\}$ is a legal shape, $x^{\prime} \geq x$. Thus if the second puncture enters column $y$ at row $p$, then $p \geq x$. So Lemma 9.4 applies. That the paths never cross is now immediate from the definition of this concept given in Section 7 . We still must address the final resting place of the punctures. The first puncture stops at the outer corner $(w, z)$ of $\lambda$. Suppose that it left column $z-1$ at row $i_{\boldsymbol{x}}$. Suppose the second puncture reaches column $z-1$. (If not, we are done.) By induction 
the second puncture reaches at least the $i_{\boldsymbol{x}}$ th row of column $z-1$. Now an easy argument (similar to the argument in the proof of Lemma 9.4 involving $\mathcal{V}_{1}, \mathcal{V}_{2}$ and Lemma 8.7) shows that the second puncture must reach at least row $w$ of column $z-1$ and thus cannot enter column $z$.

Now we prepare to verify the bijectivity claim of Theorem 7.3. Recall the action of $\sigma \in K_{4}$ on the set of admissible columns. It has the effect of rotating a (split-)column $180^{\circ}$ and then barring all the unbarred entries while unbarring all unbarred entries (see Section 8). We extend this action to the set of (punctured) admissible skew tableaux. Recall that for any shape $\lambda=\left(\lambda_{1} \geq \lambda_{2} \geq \cdots \geq \lambda_{n} \geq 0\right)$, $l(\lambda)$ is the number of non-zero entries $\lambda_{i}$. Fix a skew shape $\lambda \backslash \mu$ and fix $\mathcal{T} \in \mathcal{D}(\lambda \backslash \mu)$. Define $\sigma \mathcal{T}$ as follows. Let $\rho:=\left(\lambda_{1}^{l(\lambda)}\right)$; this is the smallest possible rectangular shape $\rho$ such that $\lambda \subset \rho$. The shapes of $\rho \backslash \lambda$ and $\rho \backslash \mu$ are respectively $180^{\circ}$ rotations of the ordinary shapes $\nu$ and $\kappa$ defined by $\nu_{i}=\lambda_{1}-\lambda_{l(\lambda)-i+1}$ and $\kappa_{i}=\lambda_{1}-\mu_{l(\lambda)-i+1}$ for $1 \leq i \leq l(\lambda)$. Form $\sigma \mathcal{T}$ from $\mathcal{T}$ by rotating the entire tableau $180^{\circ}$ and then barring all the unbarred entries while unbarring all the barred entries. It is easy to see that $\sigma \mathcal{T} \in \mathcal{D}(\kappa \backslash \nu)$. The action of $\sigma$ can also be described by: the box $(s, t)$ of $\mathcal{T}$ contains an $\overline{\mathbf{a}}, \mathbf{b}$, or is empty, if and only if the box $(l(\lambda)-s+1, \lambda-1-t+1)$ of $\sigma \mathcal{T}$ respectively contains an $\mathbf{a}, \overline{\mathbf{b}}$, or is empty. Here is an example of $\sigma$ taking an element of $\mathcal{D}\left(\left(3^{2}, 2^{2}\right) \backslash(2,1)\right)$ to an element of $\mathcal{D}\left(\left(3^{2}, 2,1\right) \backslash\left(1^{2}\right)\right)$ :

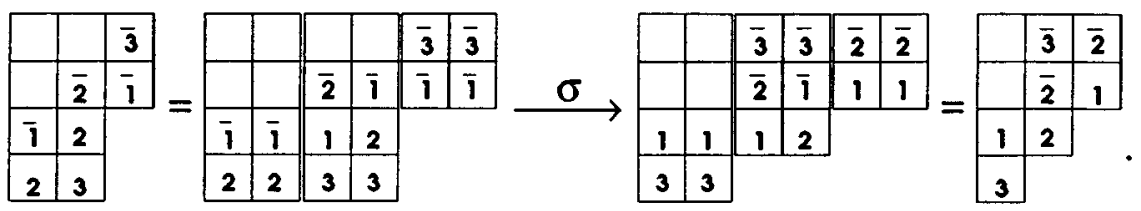

For $\mathcal{T}$ a punctured admissible skew tableau of shape $\lambda \backslash \mu$ with puncture at $(p, q)$, define $\tilde{\sigma}(\mathcal{T},(p, q))=\left(\sigma \mathcal{T},\left(l(\lambda)-p+1, \lambda_{1}-q+1\right)\right)$. Extend $\tilde{\sigma}$ to allow $(p, q)$ to be either an outer corner of $\lambda$ or an inner corner of $\mu$ : For example, if $(\mathcal{T},(p, q)) \in \mathcal{D}(\lambda \backslash \mu) \times\{$ outer corners of $\lambda\}$, set $\lambda^{\prime}=\lambda+\{(p, q)\}$ and regard $\mathcal{T}$ as a punctured tableau of shape $\lambda^{\prime} \backslash \mu$ with puncture at $(p, q)$. Then $\tilde{\sigma}(\mathcal{T},(p, q))=$ $\left(\sigma \mathcal{T},\left(l\left(\lambda_{1}^{\prime}\right)-p+1, \lambda_{1}^{\prime}-q+1\right)\right)$. The involution $\tilde{\sigma}$ is used to form the inverse of the sjdt:

Proposition 9.6. The map sjdt is a bijection with inverse $\tilde{\sigma} \mathbf{s j d t} \tilde{\sigma}$.

Proof. Recall that the sjdt is essentially the repeated application of the operator $\Pi$. We will show below that $\tilde{\sigma} \Pi \tilde{\sigma} \Pi$ is the identity operator. Since $\tilde{\sigma}^{2}$ is the identity, it then follows that $\tilde{\sigma} \mathbf{s j d t} \tilde{\sigma} \operatorname{sjdt}(\mathcal{T},(p, q))=(\mathcal{T},(p, q))$ for any $(\mathcal{T},(p, q)) \in$ $\mathcal{D}(\lambda \backslash \mu) \times\{$ inner corners of $\mu\}$. Hence $\tilde{\sigma} \mathbf{s j d t} \tilde{\sigma}$ is a left inverse of sjdt. Conjugating $\tilde{\sigma} \mathbf{s j d t} \tilde{\sigma} \mathbf{s j d t}$ by $\tilde{\sigma}$ yields that $\tilde{\sigma} \mathbf{s j d t} \tilde{\sigma}$ is also a right inverse. The proposition then follows.

Let $\rho:=\left(\lambda_{1}^{l(\lambda)}\right)$ and let $\nu$ and $\kappa$ be defined as above. So $\sigma \mathcal{T}$ is of shape $\kappa \backslash \nu$. For any box $(s, t)$ of $\rho$ let $\left(s^{\vee}, t^{\vee}\right)=\left(l(\lambda)-s+1, \lambda_{1}-t+1\right)$. Note that the entries $l_{p, q}$ and $r_{p, q}$ of the split form of a tableau $\mathcal{T}$ correspond respectively to the entries $r_{p^{\vee}, q^{\vee}}$ and $l_{p^{\vee}, q^{\vee}}$ of $\sigma \mathcal{T}$. Let $\mathcal{T}^{\prime}=\Pi(\mathcal{T},(p, q))_{1}$. Attach a prime $\left({ }^{\prime}\right)$ to any quantity associated to $\mathcal{T}^{\prime}$.

Suppose $\mathcal{T}^{\prime}=\mathbf{v}_{D}(\mathcal{T},(p, q))$. Then the puncture is at $(p+1, q)$. It is easily seen that $\mathcal{T}^{\prime}$ satisfies the condition that either $l_{p, q}^{\prime} \geq r_{p+1, q-1}^{\prime}$ or $(p+1, q-1) \notin \lambda \backslash \mu$. Note 
that the puncture of $\sigma \mathcal{T}^{\prime}$ is at $\left(p^{\vee}-1, q^{\vee}\right)$. Since the entries of $\mathcal{T}$ are "multiplied by -1 " when applying $\sigma$, the condition on $\mathcal{T}^{\prime}$ is equivalent to the following condition on $\sigma \mathcal{T}^{\prime}$ : either $r_{p^{\vee}, q^{\vee}}^{\prime} \leq l_{p^{\vee}-1, q^{\vee}+1}^{\prime}$ or $\left(p^{\vee}-1, q^{\vee}+1\right) \notin \kappa$. Thus applying $\Pi$ to $\left(\sigma \mathcal{T}^{\prime},\left(p^{\vee}-1,{ }^{q} \vee\right)\right)$ moves the puncture down to $\left(p^{\vee}, q^{\vee}\right): \Pi\left(\sigma \mathcal{T}^{\prime},\left(p^{\vee}-1, q^{\vee}\right)\right)=$ $\left(\mathbf{v}_{D}\left(\sigma \mathcal{T}^{\prime},\left(p^{\vee}-1, q^{\vee}\right)\right),\left(p^{\vee}, q^{\vee}\right)\right)=\left(\sigma \mathcal{T},\left(p^{\vee}, q^{\vee}\right)\right)$. Thus we have $\tilde{\sigma} \Pi \tilde{\sigma} \Pi(\mathcal{T},(p, q))=$ $(\mathcal{T},(p, q))$.

Now suppose $\mathcal{T}^{\prime}=\mathbf{h}_{R}(\mathcal{T},(p, q))$. Then the puncture is at $(p, q+1)$. By Lemma 9.1(1), the tableau $\mathcal{T}^{\prime}$ satisfies the condition that either $l_{p-1, q+1}^{\prime}<r_{p, q}^{\prime}$ or $(p-1, q+$ $1) \notin \lambda \backslash \mu$. The puncture of $\sigma \mathcal{T}^{\prime}$ is at $\left(p^{\vee}, q^{\vee}-1\right)$. The condition on $\mathcal{T}^{\prime}$ is equivalent to the following condition on $\sigma \mathcal{T}^{\prime}: r_{p^{\vee}+1, q^{\vee}-1}^{\prime}>l_{p^{\vee}, q^{\vee}}^{\prime}$ or $\left(p^{\vee}+1, q^{\vee}-1\right) \notin \kappa$. Thus applying $\Pi$ to $\left(\sigma \mathcal{T}^{\prime},\left(p^{\vee}, q^{\vee}-1\right)\right)$ moves the puncture to the right to $\left(p^{\vee}, q^{\vee}\right)$, i.e. $\Pi \tilde{\sigma} \Pi(\mathcal{T},(p, q))=\left(\mathbf{h}_{R} \tilde{\sigma}\left(\mathbf{h}_{R}(\mathcal{T},(p, q)),(p, q+1)\right),\left(p^{\vee}, q^{\vee}\right)\right)$. By following the definitions it is easily seen that $\mathbf{h}_{R} \tilde{\sigma}\left(\mathbf{h}_{R}(\mathcal{T},(p, q)),(p, q+1)\right)=\sigma \mathcal{T}$. Thus, again we have $\tilde{\sigma} \Pi \tilde{\sigma} \Pi(\mathcal{T},(p, q))=(\mathcal{T},(p, q))$.

Now we complete the proof of Theorem 7.3:

Proof of Parts (2) and (4) of Theorem 7.3. Half of Part (2) was established above. Using the inverse map $\tilde{\sigma} \mathbf{s j d} \mathbf{t} \tilde{\sigma}$ in conjunction with this, the rest of Part (2) is easily argued.

Part (4): By Proposition 9.2(2), the symbol $\overline{\mathbf{n}+\mathbf{1}}$ appears at $(x, y)$ of $\mathcal{S}$. For now suppose that $(x, y-1)$ is an inner corner of $\mu^{\prime}$. Let $\left(\mathcal{R},\left(w^{\prime}, z^{\prime}\right)\right)=\operatorname{sjdt}(\mathcal{S},(x, y-1))$. Since $y-1<y$, by Lemma 9.6 we have $z^{\prime}<z$ and the paths of the two punctures do not cross. The first move of this execution of sjdt will be to move the $\overline{\mathbf{n}+\mathbf{1}}$ at $(x, y)$ to the left. Let $\mathcal{E}$ denote the resulting punctured tableaux of this move. Note that the columns $y+1, y+2, \cdots$ of $\mathcal{E}$ are identical to those of $\mathcal{S}^{\prime}$. The split form of the $y$ th column of $\mathcal{E}$ is also identical to that of $\mathcal{S}^{\prime}$ in every way except for having an additional pair of symbols $\mathbf{n}+\mathbf{1}$ at its end. These symbols are treated by the sjdt just as if they were an empty box. Thus the remaining puncture path made by the application $\operatorname{sjdt}(\mathcal{S},(x, y-1))$ is identical to the puncture path made by the application $\operatorname{sjdt}\left(\mathcal{S}^{\prime},(x, y)\right)$. The result follows. Now suppose $(x, y-1)$ is not an inner corner of $\mu^{\prime}$. Temporarily replace the first $y-1$ columns of $\mathcal{T}$ (which play no part in the algorithm) with one appropriate first column whose first non-empty box is at row $x+1$. Starting with this new $\mathcal{T}$ and the inner corner $(x, 2)$ we may now apply the above argument.

\section{Proof of the tableau BiJection}

In this section we prove Theorem 7.5, which claims that the map $\Phi_{\lambda}^{n}: \mathcal{M}^{n}(\lambda, n)$ $\rightarrow \mathcal{M}^{n-1}(\lambda, n)$ defined by Algorithm 7.4 is a well defined weight preserving bijection. In Proposition 10.1 we verify that the algorithm is well defined and weight preserving. Then we propose an inverse map $\Theta_{\lambda}^{n}$ which is defined by Algorithm 10.2. In Proposition 10.3 we show that $\Theta_{\lambda}^{n}$ is a left inverse. We finish by showing that $\Theta_{\lambda}^{n}$ is also a right inverse.

Proposition 10.1. Fix $n \geq 1$ and let $\lambda$ be a shape with at most $n$ rows. Then the map $\Phi_{\lambda}^{n}: \mathcal{M}^{n}(\lambda, n) \rightarrow \mathcal{M}^{n-1}(\lambda, n)$ is well defined and weight preserving.

Proof. Let $\mathcal{T} \in \mathcal{M}^{n}(\lambda, n)$. Let $\mathcal{D}$ and $\mathcal{K}$ be as specified in Algorithm 7.4, where the shape of $\mathcal{D}$ is $\pi \backslash(k)$. Lemma 8.7 implies that $\mathcal{D}$ is admissible. It is clear that each step of Algorithm 7.4 can be carried out. It is also clear that the resulting tableaux 
$\mathcal{D}$ and $\mathcal{K}$ can be fit together to form some tableau $\mathcal{Q}$ of shape $\lambda$. So confirming well definedness consists of showing that $\mathcal{Q}$ lies in $\mathcal{M}^{n-1}(\lambda, n)$. Theorem 7.3 implies that the resulting tableau $\mathcal{D}$ lies in $\mathcal{D}(\nu, n-1)$ for some shape $\nu$. The entries of $\mathcal{K}$ are either $\overline{\mathbf{n}}$ 's or n's, and we are assuming that $\lambda$ has at most $n$ rows. Thus if $\mathcal{K}$ is semistandard then $\mathcal{Q}$ lies in $\mathcal{M}^{n-1}(\lambda, n)$ and the map $\Phi_{\lambda}^{n}$ is well defined.

We show $\mathcal{K}$ is semistandard by induction on the number of cycles of the Whileloop. The tableau $\mathcal{K}$ is trivially semistandard before the first cycle of the Whileloop. Assume $\mathcal{K}$ is semistandard before the $m$ th cycle of the While-loop. At the beginning of the $m$ th cycle we see that $(\mathcal{D},(s, t))$ results from the $m$ th application of the sjdt. Now we are required to place an $\overline{\mathbf{n}}$ in $\mathcal{K}$ at $(s, t)$. This leaves $\mathcal{K}$ semistandard since by Theorem 7.3(2) and (4), the box $(s, t)$ must be strictly to the left of all the other $\overline{\mathbf{n}}$ 's placed in $\mathcal{K}$. If an $\mathbf{n}$ and $\overline{\mathbf{n}}$ do not appear in the new $\mathcal{D}$, then $\mathcal{K}$ is semistandard after the $m$ th cycle because the If-statement was not invoked. So assume that an $\mathbf{n}$ and an $\overline{\mathbf{n}}$ appear in the $k$ th column of $\mathcal{D}$ after the $m$ th application of sjdt. The algorithm then requires us to transfer the $\mathbf{n}$ to the $k$ th column of $\mathcal{K}$. To show $\mathcal{K}$ is semistandard after this addition, we show that this column of $\mathcal{K}$ was empty beforehand. It is easy to see that this column cannot contain an $\overline{\mathbf{n}}$ : Note that the $\mathbf{n}$ and $\overline{\mathbf{n}}$ could arise in the $k$ th column of $\mathcal{D}$ only as a result of a horizontal move. So we must have $s>k$. Since the $\overline{\mathbf{n}}$ placed at $(s, t)$ is strictly to the left of all others placed in $\mathcal{K}$, the $k$ th column of $\mathcal{K}$ could not already contain an $\overline{\mathbf{n}}$. There are two possible ways an $\mathbf{n}$ could have arrived in the $k$ th column: (1) It may have been there when $\mathcal{K}$ was originally formed: (2) It may have been placed there as a result of the $(m-1)$ st cycle of the While-loop. (Note that it could not have been placed there on an earlier cycle of the While-loop because we are assuming $\mathcal{K}$ is semistandard before the $m$ th cycle: for example, if an $\mathbf{n}$ were placed in column $k$ at $(m-2)$ nd cycle, then another $\mathbf{n}$ had to have been placed there at the $(m-1)$ st cycle or else $k$ would have been decremented.) We show that assuming either (1) or (2) leads to the same contradiction.

We handle (2) first. Reset $\mathcal{D}$ and $k$ to be at the point immediately after the $(m-1)$ st application of sjdt. We refer to $\mathcal{D}$ at this point as "the old $\mathcal{D}$." By $(2)$ an $\mathbf{n}$ and $\overline{\mathbf{n}}$ have just appeared in the $k$ th column of $\mathcal{D}$. For now assume $k>1$. Then $(1, k-1)$ is an inner corner of $\mathcal{D}$. Instead of taking the $\mathbf{n}$ and $\overline{\mathbf{n}}$ out of $\mathcal{D}$, apply sjdt to $(\mathcal{D},(1, k-1))$. The first step of this application of sjdt will be a horizontal move. Let $\mathcal{E}$ be the punctured tableau resulting from this first horizontal move. Set $\mathcal{E}$ aside. Return to the old $\mathcal{D}$ and remove the $\mathbf{n}$ and $\overline{\mathbf{n}}$ so that it is now at the point just before the $m$ th application of the sjdt. Call this tableau "the new $\mathcal{D}$." The split-columns $k, k+1, \ldots$ of $\mathcal{E}$ and the new $\mathcal{D}$ are now identical except for an added pair of $\mathbf{n}$ 's at the bottom of the $k$ th split-column of $\mathcal{E}$. Thus first horizontal move of the application of sjdt to the new $(\mathcal{D},(1, k))$ and the second horizontal move in the application of sjdt to the old $(\mathcal{D},(1, k-1))$ must bring in same entry from the $(k+1)$ st to the $k$ th column. (The tableau $\mathcal{E}$ was the result of the first horizontal move of sjdt on the old $\mathcal{D}$.) Ignoring the leading empty box, let $B_{k}$ and $C_{k}$ be such that the new $\mathcal{D}_{k}=G\left(B_{k}, C_{k}\right)$. Then $\mathcal{E}_{k}=G\left(B_{k}, C_{k}+\{n\}\right)$. Recall that this horizontal move on the new $\mathcal{D}$ was assumed to result in the appearance of a second $\mathbf{n}$ and $\overline{\mathbf{n}}$ pair. By Lemma 9.1(3) the entry moved into column $k$ must be barred: For some $b$, the column $\mathcal{D}_{k}$ is replaced by $G\left(B_{k}+\{b\}, C_{k}\right)$ and the column $\mathcal{E}_{k}$ is replaced by $G\left(B_{k}+\{b\}, C_{k}+\{n\}\right)$. Since $\mathbf{n}$ and $\overline{\mathbf{n}}$ are entries in $G\left(B_{k}+\{b\}, C_{k}\right)$, the symbols $\mathbf{n}+\mathbf{1}$ and $\overline{\mathbf{n}+\mathbf{1}}$ must arise in $G\left(B_{k}+\{b\}, C_{k}+\{n\}\right)$. Since this is the second horizontal move on the old $(\mathcal{D},(1, k-1))$, we have arrived at a contradiction 
to Theorem $7.3(3)$. Hence if an $\mathbf{n}$ and $\overline{\mathbf{n}}$ appeared in the $k$ th column during an earlier iteration of the While-loop, an $\mathbf{n}$ and $\overline{\mathbf{n}}$ cannot arise in this column during the $m$ th iteration. If $k=1$, then we can temporarily attach an appropriate 0 th column and apply the same argument.

The same argument applies to case (1), where an $\mathbf{n}$ starts out in column $k$ of $\mathcal{K}$ when it was originally formed. In this case, an $\mathbf{n}$ and $\overline{\mathbf{n}}$ are in the $k$ th column of the input tableau $\mathcal{T}$. By considering the split column, it is easy to see that if the $k$ th column of an admissible $\mathcal{T}$ contains both an $\mathbf{n}$ and $\overline{\mathbf{n}}$, then there are no $\overline{\mathbf{n}}$ 's in columns $k+1, k+2, \ldots$, and there are no $\mathbf{n}$ 's in columns $1,2, \ldots, k-1$. We are therefore considering the very first cycle of the While-loop: $m=1$. Let $\mathcal{D}$ be the tableau resulting from removing all the $\mathbf{n}$ 's and $\overline{\mathbf{n}}$ 's from $\mathcal{T}$ except those in the $k$ th column. Now we can apply the argument above to show that an $\mathbf{n}$ and $\overline{\mathbf{n}}$ cannot possibly appear as a result of the 1st application of sjdt.

Aside from applying the map sjdt (which is weight preserving by Theorem 7.3), the net result of the process is to move n's and $\overline{\mathbf{n}}$ 's from $\mathcal{D}$ to $\mathcal{K}$. So it is weight preserving.

Define $\Theta_{\lambda}^{n}$ by Algorithm 10.2 below. The domain of $\Theta_{\lambda}^{n}$ is defined to be the largest subset of $\mathcal{M}^{n-1}(\lambda, n)$ for which the algorithm is well defined.

Algorithm $10.2\left(\Theta_{\infty}^{n}\right)$.

Input: A tableau $\mathcal{Q} \in \mathcal{M}^{n-1}(\lambda, n)$ with De Concini-part $\mathcal{D}$ and King-part $\mathcal{K}$.

While there are $\overline{\mathbf{n}}$ 's in $\mathcal{K}$ repeat the following two steps:

Remove the leftmost $\overline{\mathbf{n}}$ in $\mathcal{K}$ from its box $(s, t)$ and set $(\mathcal{D},(1, k))$ $:=\tilde{\sigma} \operatorname{sjdt} \tilde{\sigma}(\mathcal{D},(s, t))$.

If there is an $\mathbf{n}$ in the $k$ th-column of $\mathcal{K}$ and there are still $\overline{\mathbf{n}}$ 's in $\mathcal{K}$ then:

take this $\mathbf{n}$ from its box in $\mathcal{K}$ and place it in the corresponding box in $\mathcal{D}$ and place an $\overline{\mathbf{n}}$ in the box $(1, k)$ of $\mathcal{D}$.

Now $\mathcal{D} \in \mathcal{M}^{n-1}(\pi \backslash(k), n-1)$ for some shape $\pi$, and $\mathcal{K}$ is a skew tableaux of shape $\lambda \backslash \pi$ containing only $\mathbf{n}$ 's. Fill in the subshape $(k)$ in $\mathcal{D}$ with $\overline{\mathbf{n}}$ 's. Form $\mathcal{T}$ by putting $\mathcal{D}$ and $\mathcal{K}$ together in the obvious way.

Output: $\mathcal{T} \in \mathcal{M}^{n}(\lambda, n)$.

Proposition 10.3. The composition $\Theta_{\lambda}^{n} \Phi_{\lambda}^{n}$ is the identity on $\mathcal{M}^{n}(\lambda, n)$.

Proof. The proposition will be confirmed after we verify that Algorithm 10.2 reverses the steps of Algorithm 7.4. It is obvious that the steps before and after the While-loop of 10.2 respectively reverse the steps after and before the Whileloop of Algorithm 7.4. So we need only address the While-Loops themselves. For brevity we refer to the While-loop of Algorithm 10.2 as $W_{10.2}$, and the While-loop of Algorithm 7.4 as $W_{7.4}$.

Let $\mathcal{Q}$ be in the image of $\Phi_{\lambda}^{n}$. Let $\mathcal{D}$ and $\mathcal{K}$ be respectively the De Concini and King parts of $\mathcal{Q}$. It is clear that $W_{7.4}$ placed the $\overline{\mathbf{n}}$ 's in $\mathcal{K}$ from right to left and $W_{10.2}$ will remove them from left to right. Let $(s, t)$ be the box of the leftmost $\overline{\mathbf{n}}$ in $\mathcal{K}$. In $W_{7.4}$, the If-statement could not have been invoked during the last cycle because it leaves $\mathcal{D}$ with a skew shape while our $\mathcal{D}$ has a normal shape. As desired, the first step of $W_{10.2}$ after removing the $\overline{\mathbf{n}}$ from $\mathcal{K}$ is to apply $\tilde{\sigma}$ sjdt $\tilde{\sigma}$ to $(\mathcal{D},(s, t))$. Now we are at a general situation in $W_{10.2}$ : we have just applied the $\tilde{\sigma} \operatorname{sjdt} \tilde{\sigma}$ resulting in a new $\mathcal{D}$ with a newly created empty box at $(1, k)$. We must 
determine whether the If-statement in $W_{7.4}$ was invoked at this point. Note that if the If-statement was invoked, then an $\mathbf{n}$ must occur in column $k$ of $\mathcal{K}$. However, an $\mathbf{n}$ could also have been placed here by Algorithm 7.4 when $\mathcal{K}$ was originally formed. In this latter case, as was shown in the proof of Proposition 10.1, we are at the step before the first application of sjdt in $W_{7.4}$. Thus there are no $\overline{\mathbf{n}}$ 's in $\mathcal{K}$ and $W_{10.2}$ has finished reversing $W_{7.4}$. If there are still $\overline{\mathbf{n}}$ 's in $\mathcal{K}$ and also an $\mathbf{n}$ in column $k$ of $\mathcal{K}$, then the If-statement must have been invoked by $W_{7.4}$. As desired, before the next application of $\tilde{\sigma} \mathbf{s j d t} \tilde{\sigma}, W_{10.2}$ transfers the $\mathbf{n}$ to $\mathcal{D}$ and places an $\overline{\mathbf{n}}$ at $(1, k)$ of $\mathcal{D}$. Hence $W_{10.2}$ reverses $W_{7.4}$ and $\Theta_{\lambda}^{n} \Phi_{\lambda}^{n}$ is the identity on $\mathcal{M}^{n}(\lambda, n)$.

In the proof of Proposition 10.4 we use Theorem 7.3(2), Lemma 9.1(3) and Lemma 9.5 but as they apply to the inverse map $\tilde{\sigma} \mathbf{s j d t} \tilde{\sigma}$. We refer to them as the $\tilde{\sigma}$-conjugates of the respective results and leave the reader to form their precise statements.

Proposition 10.4. The map $\Theta_{\lambda}^{n}$ is well defined on all of $\mathcal{M}^{n-1}(\lambda, n)$.

Proof. We show that Algorithm 10.2 is well defined on $\mathcal{M}^{n-1}(\lambda, n)$ by induction on the number of cycles of the While-loop. Let $\mathcal{Q} \in \mathcal{M}^{n-1}(\lambda, n)$. Let $\mathcal{D}$ and $\mathcal{K}$ be as specified in Algorithm 10.2. We verify that immediately after the $m$ th application of $\tilde{\sigma} \operatorname{sjdt} \tilde{\sigma}, \mathcal{D}$ and $\mathcal{K}$ satisfies the following three conditions which are our inductive hypotheses:

(I) $\mathcal{D}$ is an admissible skew tableau whose empty inner shape is a single row.

Let $k$ be the length of this row.

(II) $\mathcal{K}$ has no entries in its first $k-1$ columns.

(III) The entries of $\mathcal{D}$ are from $[[\mathbf{n}-\mathbf{1}]]$.

First we check that the conditions hold for $m=1$ : Condition (I) is obvious. Condition (II) is vacuous here since $k=1$. We argue by contradiction that condition (III) is also satisfied: An $\mathbf{n}$ and $\overline{\mathbf{n}}$ can appear in $\mathcal{D}$ only as a result of a horizontal move of $\tilde{\sigma} \mathbf{s j d t} \tilde{\sigma}$. Assume this is the case and let $\mathcal{D}$ denote the admissible punctured tableau immediately after this horizontal move. It still has an ordinary non-skew shape. Since the $\overline{\mathbf{n}}$ is the smallest element of $\mathcal{D}$, it must occur in the first row. Since the $\overline{\mathbf{n}}$ resulted from a horizontal move it cannot occur in the first column. The only possibility which satisfies semistandardness is that the $\overline{\mathbf{n}}$ is at $(1,2)$ and the puncture is at $(1,1)$. Thus, the puncture went from $(1,2)$ to $(1,1)$ during this horizontal move. By the $\tilde{\sigma}$-conjugate of Lemma $9.1(3)$ the entry originally at $(1,1)$ must have been unbarred. Since the original tableaux $\mathcal{D}$ was semistandard, this implies all of its entries were unbarred. In such a tableau, a horizontal move never creates an $\mathbf{n}$ and $\overline{\mathbf{n}}$ pair. This is a contradiction.

Now let $m \geq 2$ and $1 \leq j \leq m$. Let $\mathcal{D}^{j}$ and $\mathcal{K}^{j}$ denote the tableaux $\mathcal{D}$ and $\mathcal{K}$ at the point just after the $j$ th application of $\tilde{\sigma}$ sjdt $\tilde{\sigma}$. For $1 \leq j<m$ assume that $\mathcal{D}^{j}$ and $\mathcal{K}^{j}$ satisfy (I), (II), and (III). We show that $\mathcal{D}^{m}$ and $\mathcal{K}^{m}$ satisfy these conditions as well. Let $\left(s_{j}, t_{j}\right)$ be the location of the leftmost $\overline{\mathbf{n}}$ in $\mathcal{K}^{j-1}$ (this is the $j$ th $\overline{\mathbf{n}}$ from the left of the input tableau). Let $\left(k_{j}\right)$ be the inner shape of $\mathcal{D}^{j}$. Let $k_{0}=0$ and let $\mathcal{D}^{0}$ and $\mathcal{K}^{0}$ be $\mathcal{D}$ and $\mathcal{K}$ immediately before the first application of $\tilde{\sigma} \operatorname{sjdt} \tilde{\sigma}$.

Case 1: Assume the If-statement was not invoked during the $(m-1)$ st cycle. Then $\mathcal{D}$ contains no $\mathbf{n}$ and $\overline{\mathbf{n}}$ at the point just before the $m$ th application of $\tilde{\sigma} \operatorname{sjdt} \tilde{\sigma}$. By $\tilde{\sigma}$-conjugate of Theorem 7.3(2) we know the $m$ th application of $\tilde{\sigma}$ sjdt $\tilde{\sigma}$ puts the puncture in the first row of column $k_{m}=k_{m-1}+1$. So we have (I). Since the 
If-statement was not invoked during the $(m-1)$ st cycle, there was no $\mathbf{n}$ in column $k_{m-1}$ of $\mathcal{K}^{m-1}$. Column $k_{m-1}$ of $\mathcal{K}^{m-1}$ could not have contained an $\overline{\mathbf{n}}$ since we had already taken $m-1 \overline{\mathbf{n}}$ 's out of the $\mathcal{K}$ tableau working from left to right, and from the algorithm it is obvious that $k_{m-1} \leq m-1$. Thus column $k_{m-1}$ of $\mathcal{K}^{m-1}$ was empty. This and the inductive condition (II) yield that $\mathcal{K}^{m-1}$ had no entries in its first $k_{m-1}$ columns. Thus $\mathcal{K}^{m}$ satisfies (II) since it has no entries in its first $k_{m}-1=k_{m-1}$ columns. For (III), ignore the first $k_{m-1}$ columns. Then this case becomes like the application of $\tilde{\sigma} \mathbf{s j d t} \tilde{\sigma}$ to $\mathcal{D}^{0}$ considered earlier.

Case 2: Assume the If-statement was invoked during the $(m-1)$ st cycle. Then there must have been an $\mathbf{n}$ in column $k_{m-1}$ of $\mathcal{K}^{m-1}$. We were required to transfer this $\mathbf{n}$ to $\mathcal{D}^{m-1}$ and to place an $\overline{\mathbf{n}}$ at $\left(1, k_{m-1}\right)$ of $\mathcal{D}^{m-1}$. We show that doing this results in an admissible tableau. First we verify that the box of $\mathcal{D}^{m-1}$ corresponding to the position of this $\mathbf{n}$ in $\mathcal{K}^{m-1}$ is at an outer corner: As argued in case 1, column $k_{m-1}$ of $\mathcal{K}^{m-1}$ contain no $\overline{\mathbf{n}}$ 's. From (II), $\mathcal{K}^{m-1}$ has no entries to the left of the $\mathbf{n}$ in column $k_{m-1}$. It is clear from the algorithm that at every step the tableaux $\mathcal{K}^{m-1}$ and $\mathcal{D}^{m-1}$ fit together to form an unpunctured skew tableau with outer shape $\lambda$; thus the box corresponding to this $\mathbf{n}$ is an outer corner for $\mathcal{D}^{m-1}$. Clearly the unsplit-form of the new $\mathcal{D}^{m-1}$ augmented with the added $\mathbf{n}$ and $\overline{\mathbf{n}}$ is semistandard. To show that this augmented $\mathcal{D}^{m-1}$ is admissible we form an admissible tableau $\mathcal{E}$ such that applying $\tilde{\sigma}$ sjdt $\tilde{\sigma}$ to it results in the augmented $\mathcal{D}^{m-1}$. We begin to form $\mathcal{E}$ by letting it be the tableau $\mathcal{D}$ at the point immediately before the $(m-1)$ st application of $\tilde{\sigma} \mathbf{s j d t} \tilde{\sigma}$. We claim that $\mathcal{E}$ contains no n's or $\overline{\mathbf{n}}$ 's: Suppose it does. By induction, the entries of $\mathcal{D}^{m-2}$ are from $[[\mathbf{n}-\mathbf{1}]]$. Since the tableau $\mathcal{E}$ contains an $\mathbf{n}$ and an $\overline{\mathbf{n}}$, the If-statement was invoked during the $(m-2)$ nd cycle. This implies $k_{m-2}=k_{m-1}$. The If-statement required us to take an $\mathbf{n}$ out of column $k_{m-1}$ of $\mathcal{K}^{m-2}$. This is impossible since we are assuming that there is an $\mathbf{n}$ in column $k_{m-1}$ of $\mathcal{K}^{m-1}$. Thus $\mathcal{E}$ contains no n's nor $\overline{\mathbf{n}}$ 's and so it is at this point just $\mathcal{D}^{m-2}$. Since there is an outer corner in column $k_{m-1}$ of $\mathcal{D}^{m-1}$, there must be an outer corner in column $k_{m-1}$ of $\mathcal{E}$. (This corner will either be in the same row as it is in $\mathcal{D}^{m-1}$ or in the next row down when $t_{m-1}=k_{m-1}$.) Now, to finish its formation, place an $\overline{\mathbf{n}}$ in $\mathcal{E}$ at the inner corner $\left(1, k_{m-1}-1\right)$ and place an $\mathbf{n}$ in the outer corner in column $k_{m-1}$. (If $k_{m-1}=1$, temporarily adjoin an appropriate 0th column.) Note that adding an $\overline{\mathbf{n}}$ to the top of an admissible column containing no $\mathbf{n}$ 's has the effect on the split-column of adding two $\overline{\mathbf{n}}$ 's to its top. Similarly, adding an $\mathbf{n}$ to the bottom of an admissible column containing no $\overline{\mathbf{n}}$ 's has the effect on the split-column of adding two n's to its bottom. Thus the split column form of $\mathcal{E}$ is semistandard. Apply $\tilde{\sigma} \operatorname{sjdt} \tilde{\sigma}$ to $\left(\mathcal{E},\left(s_{m-1}, t_{m-1}\right)\right)$ (or to $\left(\mathcal{E},\left(s_{m-1}+1, t_{m-1}\right)\right)$ if $\left.t_{m-1}=k_{m-1}\right)$. It is not hard to see that the result is the augmented $\mathcal{D}^{m-1}$. The admissibility of this tableau follows from the well definedness of $\tilde{\sigma} \mathbf{s j d t} \tilde{\sigma}$.

Now apply the $m$ th application of $\tilde{\sigma} \mathbf{s j d t} \tilde{\sigma}$ to the augmented $\mathcal{D}^{m-1}$ to produce $\mathcal{D}^{m}$. Note that $\mathcal{D}^{m}$ is the result of two applications of $\tilde{\sigma}$ sjdt $\tilde{\sigma}$ to $\mathcal{E}$. Thus, condition (I) follows from the $\tilde{\sigma}$-conjugate of Theorem 7.3(2). Condition (II) is satisfied since $k_{m}=k_{m-1}$. To see that $\mathcal{D}^{m}$ satisfies (III) we employ $\tilde{\sigma}$-conjugate of Lemma 9.5. The first puncture passes through column $k_{m-1}$ of $\mathcal{E}$ and the second puncture reaches this column. Let $\boldsymbol{x}$ be the entry slid out of the right half of column $k_{m-1}$ and let $\boldsymbol{z}$ be the entry slid into the left half of column $k_{m-1}$ during the first puncture's pass. Then $\boldsymbol{z}=\overline{\mathbf{n}}$. Let $\boldsymbol{x}^{\prime}$ be the entry slid out of the right half of column $k_{m-1}$ and let $\boldsymbol{y}^{\prime}$ be the entry eliminated from the left half of this column due to the second puncture's entry. Since the paths of the two punctures don't cross, $\boldsymbol{x}^{\prime} \leq \boldsymbol{x}$. 
The $\tilde{\sigma}$-conjugate of Lemma 9.5 implies $\boldsymbol{y}^{\prime} \leq \boldsymbol{z}$. Thus $\boldsymbol{y}^{\prime}=\overline{\mathbf{n}}$. Therefore the $\overline{\mathbf{n}}$ is removed from column $k_{m-1}$ by the last horizontal move of the second puncture. This implies that there are no $\overline{\mathbf{n}}^{\prime}$ s in $\mathcal{D}^{m}$. In $\mathcal{E}$ there was one $\mathbf{n}$ and one $\overline{\mathbf{n}}$. Since $\tilde{\sigma} \mathbf{j} \mathbf{j d t} \tilde{\sigma}$ is weight preserving, there are no n's in $\mathcal{D}^{m}$. Thus $\mathcal{D}^{m}$ satisfies (III), and we are done with our induction argument.

Now let $\mathcal{D}^{r}$ be the admissible tableau produced by $\tilde{\sigma} \mathbf{s j d t} \tilde{\sigma}$ during the last cycle of the While loop. Then $\mathcal{K}^{r}$ contains only n's. The If-statement was not invoked during this last cycle so (I) and (III) imply $\mathcal{D}^{r} \in \mathcal{M}^{n-1}\left(\pi \backslash\left(k_{r}\right), n-1\right)$ for some shape $\pi$. Note that there may be an $\mathbf{n}$ in column $k_{r}$ of $\mathcal{K}^{r}$. Suppose this is the case. Begin to form $\mathcal{T}$ by placing an $\overline{\mathbf{n}}$ at $\left(1, k_{r}\right)$ of $\mathcal{D}^{r}$ and transferring the $\mathbf{n}$ from column $k_{r}$ of $\mathcal{K}^{r}$ to $\mathcal{D}^{r}$. This results in an admissible tableau as was proven in the first paragraph of Case 2 in our induction argument. Now fill in the remaining inner shape of the partially formed $\mathcal{T}$ with $\overline{\mathbf{n}}$ 's and attach the remaining $\mathbf{n}$ 's of $\mathcal{K}^{r}$. By (II) there were no n's in the first $k_{r}-1$ columns of $\mathcal{K}^{r}$. The resulting $\mathcal{T}$ is admissible because we never add an $\mathbf{n}$ to a column containing an $\overline{\mathbf{n}}$. If there was no $\mathbf{n}$ in column $k_{r}$ of $\mathcal{K}^{r}$, then it is clear that forming $\mathcal{T}$ as specified also results in an admissible tableau. Thus $\mathcal{T} \in \mathcal{M}^{n}(\lambda, n)$ and therefore $\Theta_{\lambda}^{n}$ is well defined on $\mathcal{M}^{n-1}(\lambda, n)$.

Proof of Theorem 7.5. By Proposition 10.1 the map $\Theta_{\lambda}^{n}$ is well defined and weight preserving. Proposition 10.3 implies $\Theta_{\lambda}^{n}$ is injective. Below we show $\Theta_{\lambda}^{n}$ is a left inverse of $\Theta_{\lambda}^{n}$. With this, Proposition 10.4 implies $\Theta_{\lambda}^{n}$ is surjective. As in the proof of Proposition 10.3 we only show that the While-loop of Algorithm 7.4 reverses the While-loop of Algorithm 10.2; the initial and terminal details are obvious.

Let $W_{7.4}$ and $W_{10.2}$ denote the respective While-loops. Fix $\mathcal{U} \in \mathcal{M}^{n-1}(\lambda, n)$ and set $\mathcal{T}=\Theta_{\lambda}^{n}(\mathcal{U})$. Let $\mathcal{D}$ and $\mathcal{K}$ and $k$ be formed from $\mathcal{T}$ as specified at the beginning of Algorithm 7.4. It is clear that the If-statement could not have been invoked during the last cycle of $W_{10.2}$. The first step of $W_{7.4} \operatorname{sets}(\mathcal{D},(s, t)):=\operatorname{sjdt}(\mathcal{D},(1, k))$ and places an $\overline{\mathbf{n}}$ in $\mathcal{K}$ at $(s, t)$. In general, after an application of sjdt in $W_{7.4}$, if an $\mathbf{n}$ and $\overline{\mathbf{n}}$ appear in the new $\mathcal{D}$, then the If-statement must have been invoked at this point in $W_{10.2}$ : From the inductive hypothesis (III) of the proof of Proposition 10.4 it is seen that the only way an $\mathbf{n}$ and $\overline{\mathbf{n}}$ can appear in $\mathcal{D}$ during the cycles of $W_{10.2}$ is through an invokation of the If-statement. Here, $W_{7.4}$ reverses the invokation by removing the $\overline{\mathbf{n}}$ from $\mathcal{D}$ and transferring the $\mathbf{n}$ to $\mathcal{K}$. The loop $W_{7.4}$ continues to reverse $W_{10.2}$ until $\mathcal{D}$ has a normal shape and also contains no n's and $\overline{\mathbf{n}}$ 's. Thus $\Theta_{\lambda}^{n}(\mathcal{T})=\mathcal{U}$ and so $\Theta_{\lambda}^{n} \Phi_{\lambda}^{n}$ is the identity on $\mathcal{M}^{n-1}(\lambda, n)$.

\section{Conjectures}

In this section we give four conjectures concerning the symplectic jeu de taquin (sjdt): The first conjecture concerns the "global" well definedness of the sjdt; the second addresses Knuth relations for the sjdt; next we propose a bijective proof of Littelmann's rule for $\operatorname{sp}(2 n, \mathbb{C})$ tensor products; the last conjecture concerns the sets $\mathcal{D}(\lambda \backslash \mu, n)$ of admissible skew tableaux.

Global well definedness for the original jeu de taquin (jdt) concerns the process of sliding out all of the empty boxes from the inner shape of a given semistandard skew tableau. That the $\mathbf{j d t}$ is globally well defined refers to the fact that the final non-skew tableau is independent of the order in which the boxes of the inner shape are chosen. We now describe the analogous procedure for the sjdt. 
Algorithm 11.1.

Input: An admissible skew tableau $\mathcal{T}$ of shape $\lambda \backslash \mu$ with entries from [[n]]. Repeatedly apply the following two steps until neither step is possible. If at any point both steps are possible, choose either one to be the next step.

(1) If $\mu \neq \varnothing$, choose an inner corner $(i, j)$ of $\mu$ and apply the sjdt to $(\mathcal{T},(i, j))$.

(2) If there are symbols in $\mathcal{T}$ that are not in $[[\mathbf{n}]]$, remove them.

Output: A normal shaped admissible tableau with entries from [[n]].

Global well definedness for the sjdt means that for each fixed input tableau, Algorithm 11.1 results in a unique output tableau independent of the sequence of choices made during the algorithm.

Conjecture 11.2. The sjdt is globally well defined.

Using K. Eriksson's strong convergence property for the ordinary jeu de taquin [E], we have reduced this problem to the cases of skew shapes of form $\lambda \backslash \mu$ where $\lambda$ is rectangular and $\mu=(2,1)$. We have partially checked one such case by computer: When removing any $\mathbf{n}+\mathbf{1}, \overline{\mathbf{n}+\mathbf{1}}$ pairs immediately after they appear, each of two sliding out orders for each of the 47,040 elements of $\mathcal{D}\left(\left(4^{3}\right) \backslash(2,1), 4\right)$ produce the same result. In addition we have a separate proposed outline of a proof of this conjecture based upon combinatorial constructions arising in [LLT] and [KN].

Now we consider Knuth relations. Let $W^{[\mathbf{n}]}$ be the set of words on the alphabet $[\mathbf{n}]:=\{\mathbf{1}<\mathbf{2}<\cdots<\mathbf{n}\}$. The $\mathbf{j d t}$ is used to form a map from $W^{[\mathbf{n}]}$ to the set of semistandard tableaux with entries from $[\mathbf{n}]$ as follows: Let $w=w_{1} w_{2} \cdots w_{k}$. Form the skew tableau with outside shape $(k, k-1, \ldots, 1)$ whose only non-empty boxes consist of $w_{i}$ in the bottom box of the $i$ th column, $i=1, \ldots, k$. Now use the jdt to slide out the empty boxes from this skew tableau to obtain the semistandard tableau $P_{\mathbf{j d t}}(w)$. Let $P_{\mathbf{j d t}}$ define an equivalence relation: $v \equiv w \Leftrightarrow P_{\mathbf{j d t}}(v)=P_{\mathbf{j d t}}(w)$ for $v, w \in W^{[\mathbf{n}]}$. Knuth [Knu] showed that this equivalence is generated by the relations $\mathbf{b a c} \equiv \mathbf{b c a}$ for $\mathbf{a}<\mathbf{b} \leq \mathbf{c}$, and $\mathbf{a c b} \equiv \mathbf{c a b}$ for $\mathbf{a} \leq \mathbf{b}<\mathbf{c}$. These are called the Knuth relations on $W^{[\overline{\mathbf{n}}]}$. In an analogous manner we can use Algorithm 11.1 to define a map $w \mapsto P_{\text {sjdt }}(w)$ from the set $W^{[[\mathbf{n}]]}$ of words on the alphabet $[[\mathbf{n}]]$ to the set of admissible tableaux with entries from $[[\mathbf{n}]]$. In [LLT], Lascoux, Leclerc, and Thibon proposed some symplectic analogs to the Knuth relations on $W^{[[\mathbf{n}]]}$ by appealing to the crystal graphs of representations of $\operatorname{sp}(2 n, \mathbb{C})$. We conjecture that these relations generate the equivalence $v \equiv w \Leftrightarrow P_{\mathbf{s j d t}}(v)=P_{\mathbf{s j d t}}(w)$ for $v, w \in W^{[[\mathbf{n}]]}$. Here, $A_{\mathcal{P}}, B_{\mathcal{P}}, C_{\mathcal{P}}, D_{\mathcal{P}}$ are the associated subsets of the admissible column $\mathcal{P}$ (defined in Section 4 ).

Conjecture 11.3. Fix $n \geq 1$. The equivalence $v \equiv w \Leftrightarrow P_{s j d t}(v)=P_{s j d t}(w)$ for $v, w \in W^{[[\mathbf{n}]]}$ is generated by the following relations:

(I) For $\|\boldsymbol{x}\| \neq\|\boldsymbol{z}\|, \mathbf{y x z} \equiv \boldsymbol{y} \boldsymbol{z x}$ where $\boldsymbol{x}<\boldsymbol{y} \leq \boldsymbol{z}$, and $\boldsymbol{x} \boldsymbol{z y} \equiv \boldsymbol{z x} \boldsymbol{y}$ where $\boldsymbol{x} \leq \boldsymbol{y}<\boldsymbol{z}$.

(II) For $\|\boldsymbol{y}\| \leq a \leq n-1, \boldsymbol{y} \mathbf{a} \overline{\mathbf{a}} \equiv \mathbf{y} \overline{\mathbf{a}+\mathbf{1}} \mathbf{a}+\mathbf{1}$ and $\mathbf{a} \overline{\mathbf{a}} \mathbf{y} \equiv \overline{\mathbf{a}+\mathbf{1}} \mathbf{a}+\mathbf{1} \mathbf{y}$.

(III) For $w=w_{1} w_{2} \cdots w_{k}$ with $w_{1}<w_{2}<\cdots w_{k}$, let $\mathcal{P}=G\left(B_{\mathcal{P}}, C_{\mathcal{P}}\right)$ be the unique admissible column such that $F\left(B_{\mathcal{P}}, C_{\mathcal{P}}\right)$ has precisely the entries $w_{1}, w_{2}$, $\ldots, w_{k}$. Let $\mathcal{Q}$ be the column obtained by removing all entries from (the unsplit form of) $\mathcal{P}$ which are not in $[[\mathbf{n}]]$. Let $v=v_{1} v_{2} \cdots v_{k-2 m}$ where $v_{1}<v_{2}<\cdots<v_{k-2 m}$ are the entries of $F\left(B_{\mathcal{Q}}, C_{\mathcal{Q}}\right)$. Then $w \equiv v$. 
Assuming global well-definedness, relations (I) and (II) can be deduced by applying Algorithm 11.1 to tableaux of shape $(3,2,1) \backslash(2,1)$. Relation (III) comes from step (2) of Algorithm 11.1.

The solution of the tensor product problem for polynomial representations of $\operatorname{gl}(n, \mathbb{C})$ is given by the Littlewood-Richardson Rule: $\operatorname{gl}_{n}(\mu ; x) \operatorname{gl}_{n}(\nu ; x)=$ $\sum_{\lambda} c_{\mu, \nu}^{\lambda} \mathrm{gl}_{n}(\lambda ; x)$. A bijective proof using the $\mathbf{j d t}$ goes roughly as follows (e.g. [Pro2]). The character $\operatorname{gl}_{n}(\lambda ; x) \operatorname{gl}_{n}(\nu ; x)$ is the weight generating function for the set product $\mathcal{T}(\mu, n) \times \mathcal{T}(\nu, n)$. For each pair of tableaux $\left(\mathcal{T}_{\mu}, \mathcal{T}_{\nu}\right)$ in $\mathcal{T}(\mu, n) \times$ $\mathcal{T}(\nu, n)$ form a skew tableau by placing $\mathcal{T}_{\mu}$ below and to the left of $\mathcal{T}_{\nu}$ producing a $\mu_{1} \times l(\nu)$ rectangular inner shape $\rho$ of empty boxes. Call the outer shape $\kappa$. Then $\operatorname{gl}_{n}(\mu ; x) \operatorname{gl}_{n}(\nu ; x)$ is the weight generating function for the set $\mathcal{T}(\kappa \backslash \rho, n)$. Next, use the jeu de taquin on each element of $\mathcal{T}(\kappa \backslash \rho, n)$ to slide out all the empty boxes of $\rho$. Using certain properties of the jeu de taquin, it can be shown that this procedure results in a multiset $\bigcup_{\lambda} \bigcup_{c_{\mu, \nu}^{\lambda}} \mathcal{T}(\lambda, n)$ which generates the right hand side of the equation above.

Now consider the tensor product problem for $\operatorname{sp}(2 n, \mathbb{C})$. Let $n, \mu, \nu, \kappa, \rho$ be as they were for the $\operatorname{gl}(n, \mathbb{C})$ case. Consider carrying out the procedure described there with Algorithm 11.1 on the set $\mathcal{D}(\kappa \backslash \rho, n)$. We hope that a combinatorial tableau proof of Littelmann's rule $\operatorname{sp}_{2 n}(\mu ; x) \operatorname{sp}_{2 n}(\nu ; x)=\sum_{\lambda} d_{\mu, \nu}^{\lambda} \operatorname{sp}_{2 n}(\lambda ; x)$ may be produced in this way. The main ingredient in such a proof would be Conjecture 11.2 .

Conjecture 11.4. Let $n, \mu, \nu, \kappa, \rho$, and $d_{\mu, \nu}^{\lambda}$ be as above. A weight preserving bijection from $\mathcal{D}(\kappa \backslash \rho, n)$ to the multiset $\bigcup_{\lambda} \bigcup_{d_{\mu, \nu}^{\lambda}} \mathcal{D}(\lambda, n)$ is obtained by applying Algorithm 11.1 to each tableau in $\mathcal{D}(\kappa \backslash \rho, n)$.

In Section 4 we defined the sets of signed skew tableau $\mathcal{D}(\lambda \backslash \mu, n)$. The definition seems to be a natural extension of the combinatorial definition of the set $\mathcal{T}(\lambda \backslash \mu, n)$ of semistandard skew tableaux with entries from $\{\mathbf{1}, \mathbf{2}, \ldots, \mathbf{n}\}$. The question is: What are the weight generating functions of $\mathcal{D}(\lambda \backslash \mu, n)$ ?

We review the general linear case first. Fix $n, k>0$ and a shape $\lambda$ with no more than $k+n$ rows. There is a natural algebraic notion of "skew character" which arises from the algebra restriction $\operatorname{gl}(k+n, \mathbb{C}) \downarrow \operatorname{gl}(k, \mathbb{C}) \times \operatorname{gl}(n, \mathbb{C})$. The character $\operatorname{gl}_{(k+n)}(\lambda ; x)$ decomposes into a sum of $\operatorname{gl}(k, \mathbb{C}) \times \operatorname{gl}(n, \mathbb{C})$ characters: $\operatorname{gl}_{(k+n)}\left(\lambda ; x_{1}, \ldots, x_{k}, y_{1}, \ldots, y_{n}\right)=\sum \operatorname{gl}_{k}(\mu ; x) \operatorname{gl}_{n}(\lambda \backslash \mu ; y)$, where the sum is over all shapes $\mu \subset \lambda$. For fixed $\mu$ this defines the skew character $\operatorname{gl}_{n}(\lambda \backslash \mu ; y)$. It is well known that $\operatorname{gl}_{n}(\lambda \backslash \mu ; y)$ is the weight generating function for $\mathcal{T}(\lambda \backslash \mu, n)$. In general, skew characters are reducible: $\operatorname{gl}_{n}(\lambda \backslash \mu ; y)=\sum_{\nu} c_{\mu, \nu}^{\lambda} \mathrm{gl}_{n}(\nu ; y)$. Further, the jdt yields a weight preserving bijection from $\mathcal{T}(\lambda \backslash \mu, n)$ to $\bigcup_{\nu} \bigcup_{c_{\mu, \nu}^{\lambda}} \mathcal{T}(\nu, n)$.

Now we consider the situation for $\operatorname{sp}(2 n, \mathbb{C})$. Fix $k, n>0$ and a shape $\lambda$ with no more than $k+n$ rows. In this case the skew character depends on $k$ : $\operatorname{sp}_{2(k+n)}\left(\lambda ; x_{1}, \ldots, x_{k}, y_{1}, \ldots, y_{n}\right)=\sum \operatorname{sp}_{2 k}(\mu ; x) \operatorname{sp}_{2 k, 2 n}(\lambda \backslash \mu ; y)$. Let $F_{n}(\lambda \backslash \mu ; y)$ be the weight generating function for $\mathcal{D}(\lambda \backslash \mu, n)$. There is no hope that $F_{n}(\lambda \backslash \mu ; y)$ equals $\operatorname{sp}_{2 k, 2 n}(\lambda \backslash \mu ; y)$ since the former lacks the parameter $k$. However, after computing several examples we conjecture that not only is $F_{n}(\lambda \backslash \mu ; y)$ a character, but so is $\operatorname{sp}_{2 k, 2 n}(\lambda \backslash \mu ; y)-F_{n}(\lambda \backslash \mu ; y)$.

Conjecture 11.5. Fix $k, n \geq 1$ and let $\mu \subset \lambda$ be shapes with no more than $k+n$ rows. Let $V_{2 k, 2 n}(\lambda \backslash \mu)$ be an $\operatorname{sp}(2 n, \mathbb{C})$-module with character $\operatorname{sp}_{2 k, 2 n}(\lambda \backslash \mu ; y)$. Then there exists a submodule $W \subseteq V_{2 n, 2 k}(\lambda \backslash \mu)$ with character $F_{n}(\lambda \backslash \mu ; y)$. If 
$F_{n}(\lambda \backslash \mu ; y)=\sum_{\nu} e_{\mu, \nu}^{\lambda} \operatorname{sp}_{2 n}(\nu ; y)$, then a weight preserving bijection from $\mathcal{D}(\lambda \backslash \mu, n)$ to the multiset $\bigcup_{\nu} \bigcup_{e_{\mu, \nu}^{\lambda}} \mathcal{D}(\nu, n)$ is obtained by applying Algorithm 11.1 to each tableau in $\mathcal{D}(\lambda \backslash \mu, n)$.

\section{APPENDIX}

We relate the definition from $[\mathrm{DeC}]$ of admissible tableaux used in this paper to the definition of symplectic tableaux from [LMS, Lit1] and to another from [KN]. We first show that the definition from [LMS, Lit1] is just another way of defining the split-column form of an admissible tableau. Then we show that the tableaux from $[\mathrm{KN}]$ are obtained by collapsing the split column forms of admissible tableaux down to coadmissible columns rather than admissible columns. This connection was first proved by Lebris, a student of Leclerc.

Fix $0 \leq k \leq n$. Let $\mathcal{E}\left(\left(1^{k}\right), n\right)$ be the set of all $2 \times n$ circle diagrams with $k$ circles and no full slots. Then $\mathcal{E}\left(\left(1^{k}\right), n\right)$ has an induced partial order as a set of semistandard columns: $\mathcal{P} \leq \mathcal{P}^{\prime}$ iff $\mathcal{P}$ can stand to the left of $\mathcal{P}^{\prime}$ in some semistandard tableau. Let $s_{i}, 1 \leq i \leq n-1$, act on these circle diagrams by interchanging the contents of the $i$ th and $(i+1)$ st slots. In the example below $n=k=3$ and $i=1$.

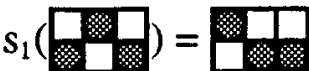

Fix $\mathcal{P}$ in $\mathcal{E}\left(\left(1^{k}\right), n\right)$ and $1 \leq i<n$. We call the action $\mathcal{P} \rightarrow s_{i}(\mathcal{P})$ a double jump if (1) $s_{i}$ moves two circles, and (2) $\mathcal{P} \leq s_{i}(\mathcal{P})$. It is easily seen that the action $\mathcal{P} \rightarrow s_{i}(\mathcal{P})$ is a double jump if and only if the $i$ th and $(i+1)$ st slots of $\mathcal{P}$ are as on the left below. Then the $i$ th and $(i+1)$ st slots of $s_{i}(\mathcal{P})$ are as below right.

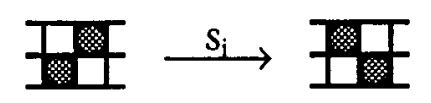

Now we state the definition of an admissible pair given in [LMS, Lit1] with this circle diagram language. A pair of columns $\left(\mathcal{Q}, \mathcal{Q}^{\prime}\right)$ is an admissible pair if and only if $\left(\mathcal{Q}, \mathcal{Q}^{\prime}\right)$ is in $\mathcal{E}\left(\left(1^{k}\right), n\right) \times \mathcal{E}\left(\left(1^{k}\right), n\right)$ for some $k \leq n$, and either $\mathcal{Q}=\mathcal{Q}^{\prime}$ or there exists a sequence of circle diagrams $\mathcal{Q}=\mathcal{Q}_{1}, \mathcal{Q}_{2}, \ldots, \mathcal{Q}_{m}=\mathcal{Q}^{\prime}$ and integers $i_{1}, i_{2}, \ldots, i_{m-1}$ with $1 \leq i_{j} \leq n-1$, such that $s_{i_{j}}\left(\mathcal{Q}_{j-1}\right)=\mathcal{Q}_{j}$ and $\mathcal{Q}_{j-1} \rightarrow \mathcal{Q}_{j}$ is a double jump for $1 \leq j \leq m$. Fix $n \geq 1$ and a shape $\lambda$ with no more than $n$ rows. Let $\mathcal{T}$ be a signed tableau of shape $\left(2 \lambda_{1}, 2 \lambda_{2}, \ldots, 2 \lambda_{n}\right)$ with columns $\mathcal{T}_{1}, \mathcal{T}_{2}, \ldots, \mathcal{T}_{2 \lambda_{1}}$ and entries from $[[\mathbf{n}]]$. Then $\mathcal{T} \in \mathcal{L} \mathcal{M S}(\lambda, n)$ if and only if (i) $\left(\mathcal{T}_{2 i-1}, \mathcal{T}_{2 i}\right)$ is an admissible pair for $1 \leq i \leq \lambda_{1}$, and (ii) $\mathcal{T}_{2 i} \leq \mathcal{T}_{2 i+1}, 1 \leq i<\lambda_{1}$.

Theorem A.1. The set $\mathcal{L} \mathcal{M S}(\lambda, n)$ is the set of tableaux $\mathcal{D}(\lambda, n)$ in split column form.

Proof. Fix $n \geq k \geq 1$. Let $\mathcal{A P}(k, n)$ be the subset of admissible pairs $\left(\mathcal{Q}, \mathcal{Q}^{\prime}\right)$ in $\mathcal{E}\left(\left(1^{k}\right), n\right)^{2}$. By comparing the definition above to the definition of $\mathcal{D}(\lambda, n)$ in Section 4 it is clear that we need only show that $\mathcal{A P}(k, n)=\{(l \mathcal{T}, r \mathcal{T}) \mid \mathcal{T} \in$ $\left.\mathcal{D}\left(\left(1^{k}\right), n\right)\right\}$. First we show that when $k=n$ both sets are equal to the set $\mathcal{B}(n)=\left\{\left(\mathcal{P}, \mathcal{P}^{\prime}\right) \mid\left(\mathcal{P}, \mathcal{P}^{\prime}\right) \in \mathcal{E}\left(\left(1^{n}\right), n\right)^{2}, \mathcal{P} \leq \mathcal{P}^{\prime}\right.$, and the number of circles in the top row of $\mathcal{P}$ is same as the number of those in $\left.\mathcal{P}^{\prime}\right\}$.

It is clear that $\left\{(l \mathcal{T}, r \mathcal{T}) \mid \mathcal{T} \in \mathcal{D}\left(\left(1^{n}\right), n\right)\right\} \subseteq \mathcal{B}(n)$. Fix $\left(\mathcal{P}, \mathcal{P}^{\prime}\right) \in \mathcal{B}(n)$ and suggestively denote by $A, B, C, D$ the subsets of $[n]$ such that $\mathcal{P}=F(A, C)$ and 
$\mathcal{P}^{\prime}=F(B, D)$. We show $\mathcal{T}=F(A, D)$ is admissible: Note that since in $\mathcal{P}$ and $\mathcal{P}^{\prime}$ we have as many circles as slots and no full slots we have $C=[n]-A$ and $D=[n]-B$. Since $\mathcal{P} \leq \mathcal{P}^{\prime}$ we have $B \leq A$. This implies $B-(A \cap B) \leq A-(A \cap B)$ which implies $B \cap([n]-A) \leq A \cap([n]-B)$. Thus $J:=B \cap C \leq A \cap D=: I$. So $F(A, D)$ is admissible. Since there are as many slots as circles we have no choice but to have $l \mathcal{T}=F(A, C)=\mathcal{P}$ and $r \mathcal{T}=F(B, D)=\mathcal{P}^{\prime}$. Hence $\{(l \mathcal{T}, r \mathcal{T}) \mid \mathcal{T} \in$ $\left.\mathcal{D}\left(\left(1^{n}\right), n\right)\right\}=\mathcal{B}(n)$.

Since the $s_{i}$ 's preserve the number of top row circles, it is clear that $\mathcal{A P}(k, n) \subseteq$ $\mathcal{B}(n)$. Note that any $\mathcal{P} \in \mathcal{E}\left(\left(1^{n}\right), n\right)$ is determined by the circles in its bottom row. Since all the slots of $\mathcal{P}$ are half-full, if the bottom row of $\mathcal{P}$ has a circle in slot $i$ and no circle in slot $i+1$, then $\mathcal{P} \rightarrow s_{i}(\mathcal{P})$ is a double jump. This double jump has the effect on the bottom row of $\mathcal{P}$ of sliding the circle in slot $i$ one slot to its right. Fix $\left(\mathcal{P}, \mathcal{P}^{\prime}\right)$ in $\mathcal{B}(n)$. To get a sequence of double jumps from $\mathcal{P}$ to $\mathcal{P}^{\prime}$ form a list of the one slot slides required to move the rightmost circle in the bottom row of $\mathcal{P}$ to the slot of the rightmost circle in the bottom row of $\mathcal{P}^{\prime}$. Next, append to the end of this list the one slot slides required to move the circle second from right in the bottom row of $\mathcal{P}$ to the slot of the corresponding circle of $\mathcal{P}^{\prime}$. Continue for each of the circles in the bottom row of $\mathcal{P}$ working from right to left resulting in the list $\left\{i_{1}, i_{2}, \ldots, i_{z}\right\}$. Then we have $s_{i_{z}} \cdots s_{i_{2}} s_{i_{1}}(\mathcal{P})=\mathcal{P}^{\prime}$. Hence $\left(\mathcal{P}, \mathcal{P}^{\prime}\right)$ is an admissible pair and $\mathcal{A P}(n, n)=\mathcal{B}(n)$.

Now fix $1 \leq k \leq n$. We show that $\mathcal{A P}(k, n)=\left\{(l \mathcal{T}, r \mathcal{T}) \mid \mathcal{T} \in \mathcal{D}\left(\left(1^{k}\right), n\right)\right\}$. For $\mathcal{P} \in \mathcal{E}\left(\left(1^{k}\right), n\right)$ let $\bar{U}(\mathcal{P})$ denote the set of slots of $\mathcal{P}$ containing a circle. Note that if $\mathcal{P} \rightarrow s_{i}(\mathcal{P})$ is a double jump then $U(\mathcal{P})=U\left(s_{i}(\mathcal{P})\right)$. Fix an admissible pair $\left(\mathcal{Q}, \mathcal{Q}^{\prime}\right)$ in $\mathcal{A P}(k, n)$. Then $U(\mathcal{Q})=U\left(\mathcal{Q}^{\prime}\right)$. Set $\left\{b_{1}<b_{2}<\cdots<b_{\nu}\right\}:=[n]-U(Q)$. Partition the circle diagrams $\mathcal{Q}$ and $\mathcal{Q}^{\prime}$ into runs of slots such that each part is either without circles or it corresponds to the slots of a run $\left\{b_{i}+1, b_{i}+2, \ldots, b_{i+1}-1\right\}$ in $U(\mathcal{Q})=U\left(\mathcal{Q}^{\prime}\right)$ :

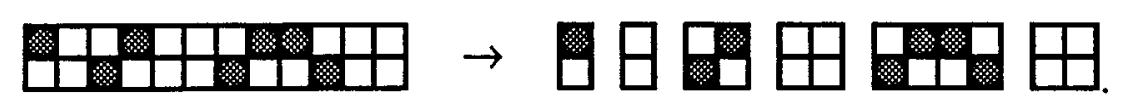

Let $\mathcal{R}$ be any part of $\mathcal{Q}$ containing circles. Let $\mathcal{R}^{\prime}$ be the corresponding part of $\mathcal{Q}^{\prime}$. Since the sequence of double jumps from $\mathcal{Q}$ to $\mathcal{Q}^{\prime}$ preserves the partition, a subsequence of these double jumps takes the part $\mathcal{R}$ to the part $\mathcal{R}^{\prime}$. Thus $\left(\mathcal{R}, \mathcal{R}^{\prime}\right)$ is an element of $\mathcal{A P}(s, s)$ for some $1 \leq s \leq n$. Hence $\left(\mathcal{R}, \mathcal{R}^{\prime}\right) \in\{(l \mathcal{T}, r \mathcal{T}) \mid \mathcal{T} \in$ $\left.\mathcal{D}\left(\left(1^{s}\right), s\right)\right\}$. Now glue the parts back together. Now it is easily seen that $\left(\mathcal{Q}, \mathcal{Q}^{\prime}\right)$ is in $\left\{(l \mathcal{T}, r \mathcal{T}) \mid \mathcal{T} \in \mathcal{D}\left(\left(1^{k}\right), n\right)\right\}$. This partitioning process is reversible: Fix $\mathcal{T}$ in $\mathcal{D}\left(\left(1^{k}\right), n\right)$. Let $A, B, C, D$ be the associated subsets of $\mathcal{T}$. Then $l \mathcal{T}=F(A, C)$ and $r \mathcal{T}=F(B, D)$. Let $U=A \cup B \cup C \cup D$. Recall from Section 8 that $A+C=U=$ $B+D$. thus $U(l \mathcal{T})=U=U(r \mathcal{T})$. This implies we can partition the circle diagrams for $l \mathcal{T}$ and $r \mathcal{T}$ as we did $\mathcal{Q}$ and $\mathcal{Q}^{\prime}$ above. Lemma 8.2(1) implies that for each corresponding pair of parts with circles $\left(\mathcal{S}, \mathcal{S}^{\prime}\right)$ we have $\left(\mathcal{S}, \mathcal{S}^{\prime}\right) \in\{(l \mathcal{T}, r \mathcal{T}) \mid \mathcal{T} \in$ $\left.\mathcal{D}\left(\left(1^{t}\right), t\right)\right\}$ for some $1 \leq t \leq n$. Thus $\left(\mathcal{S}, \mathcal{S}^{\prime}\right) \in \mathcal{A} \mathcal{P}(t, t)$ and there must be a sequence of double jumps from $\mathcal{S}$ to $\mathcal{S}^{\prime}$. Now glue the parts back together and concatenate the respective sequences of double jumps. It follows that $(l \mathcal{T}, r \mathcal{T}) \in$ $\mathcal{A P}(k, n)$.

Now we define the symplectic tableaux from $[\mathrm{KN}]$. As before for two semistandard columns $\mathcal{P}, \mathcal{Q}$, we write $\mathcal{P} \leq \mathcal{Q}$ if and only if $\mathcal{P}$ can stand to the left of $\mathcal{Q}$ in some semistandard tableau. For two admissible columns $\mathcal{P}, \mathcal{Q}$ write $\mathcal{P} \leq_{\mathcal{D}} \mathcal{Q}$ if and 
only if $\mathcal{P}$ can stand to the left of $\mathcal{Q}$ in some admissible tableau. For two columns $\mathcal{P}=\left\{\mathbf{x}_{i}\right\}, \mathcal{Q}=\left\{\mathbf{y}_{i}\right\}$ write $\mathcal{P} \leq_{\mathcal{K N}} \mathcal{Q}$ if and only if we have (I) $\mathcal{P} \leq \mathcal{Q}$ and (II) for all $1 \leq b \leq a$ such that there exists $p \leq q<r \leq s$ where $\mathbf{x}_{p}=\overline{\mathbf{a}}$ and $\mathbf{y}_{s}=\mathbf{a}$, and either: $\mathbf{x}_{q}=\overline{\mathbf{b}}$ and $\mathbf{x}_{r}=\mathbf{b}$, or $\mathbf{y}_{q}=\overline{\mathbf{b}}$ and $\mathbf{y}_{r}=\mathbf{b}$; then $(q-p)+(s-r)<a-b$. Fix $n \geq 1$ and a shape $\lambda$ with no more than $n$ rows. Let $\mathcal{T}$ be a signed tableau of shape $\lambda$ with columns $\mathcal{T}_{1}, \mathcal{T}_{2}, \ldots, \mathcal{T}_{\lambda_{1}}$ and entries from [[n]]. Then $\mathcal{T} \in \mathcal{K} \mathcal{N}(\lambda, n)$ if and only if (i) the $2 \times n$ circle diagrams for $\mathcal{T}_{1}, \mathcal{T}_{2}, \ldots, \mathcal{T}_{\lambda_{1}}$ are coadmissible and (ii) $\mathcal{T}_{i} \leq \mathcal{K N N}_{\mathcal{N}} \mathcal{T}_{i+1}$ for $1 \leq i<\lambda_{1}$.

In order to relate $\mathcal{K} \mathcal{N}(\lambda, n)$ and $\mathcal{D}(\lambda, n)$ we use the following result from $[\mathrm{KN}]$. The reader is referred to the original paper for its proof.

Lemma A.2 $([\mathrm{KN}, 4.4 .2])$. Let $\mathcal{P}=\left\{\mathbf{x}_{i}\right\}$ and $\mathcal{Q}=\left\{\mathbf{y}_{i}\right\}$ be coadmissible columns with $\mathcal{P} \leq_{\mathcal{K N}} \mathcal{Q}$. Suppose $\mathbf{x}_{p}=\overline{\mathbf{a}}$ and $\mathbf{y}_{s}=\mathbf{a}^{\prime}$, and either: $\mathbf{x}_{q}=\overline{\mathbf{b}}$ and $\mathbf{x}_{r}=\mathbf{b}^{\prime}$, or $\mathbf{y}_{q}=\overline{\mathbf{b}}$ and $\mathbf{y}_{r}=\mathbf{b}^{\prime}$ where $1 \leq b \leq a, 1 \leq b^{\prime} \leq a^{\prime}$, and $p \leq q<r \leq s$. Then $(q-p)+(s-r)<\max \left(a, a^{\prime}\right)-\min \left(b, b^{\prime}\right)$.

We verify that $\leq_{\mathcal{K N}}$ is transitive since this is not obvious from the definition.

Lemma A.3. If $\mathcal{P} \leq_{\mathcal{K N}} \mathcal{Q}$ and $\mathcal{Q} \leq_{\mathcal{K N}} \mathcal{R}$ then $\mathcal{P} \leq_{\mathcal{K N}} \mathcal{R}$.

Proof. Let $\mathcal{P}=\left\{\mathbf{x}_{i}\right\}, \mathcal{Q}=\left\{\mathbf{y}_{i}\right\}$, and $\mathcal{R}=\left\{\mathbf{z}_{i}\right\}$. Assume $\mathcal{P} \not \mathcal{K N}_{\mathcal{N}} \mathcal{R}$. It is clear that $\mathcal{P} \leq \mathcal{R}$. Thus there must exist $1 \leq b \leq a$ and $p \leq q<r \leq s$ with $\mathbf{x}_{p}=\overline{\mathbf{a}}$ and $\mathbf{z}_{s}=\mathbf{a}$, and either: $\mathbf{x}_{q}=\overline{\mathbf{b}}$ and $\mathbf{x}_{r}=\mathbf{b}$, or $\mathbf{z}_{q}=\overline{\mathbf{b}}$ and $\mathbf{z}_{r}=\mathbf{b}$; and $(q-p)+(s-r) \geq a-b$. Assume $\mathbf{x}_{q}=\overline{\mathbf{b}}$ and $\mathbf{x}_{r}=\mathbf{b}$. Since $\mathcal{Q} \leq \mathcal{R}$, we have $\left\|\mathbf{y}_{s}\right\| \leq\left\|\mathbf{z}_{s}\right\|=a$. This contradicts $\mathcal{P} \leq \mathcal{K N N}_{\mathcal{N}} \mathcal{Q}$ which by Lemma A.2 implies $(q-p)+(s-r)<\max \left(a,\left\|\mathbf{y}_{s}\right\|\right)-b$. A similar argument results in a contradiction of $\mathcal{Q} \leq_{\mathcal{K N}} \mathcal{R}$ when $\mathbf{z}_{b}=\overline{\mathbf{b}}$ and $\mathbf{z}_{r}=\mathbf{b}$ is assumed.

Theorem A.4. Fix $n \geq 1$ and let $\lambda$ be a shape with $l(\lambda) \leq n$. Let $\mathcal{T}$ be a tableau of shape $\lambda$ with admissible columns $\mathcal{T}_{1}=G\left(B_{1}, C_{1}\right), \ldots, \mathcal{T}_{\lambda_{1}}=G\left(B_{\lambda_{1}}, C_{\lambda_{1}}\right)$, where $B_{1}, \ldots, B_{\lambda_{1}} \subseteq[n]$ and $C_{1}, \ldots, C_{\lambda_{1}} \subseteq[\mathbf{n}]$. Form the tableau $\mathcal{S}$ of shape $\lambda$ with coadmissible columns $\mathcal{S}_{1}=F\left(B_{1}, C_{1}\right), \ldots, \mathcal{S}_{\lambda_{1}}=F\left(B_{\lambda_{1}}, C_{\lambda_{1}}\right)$. Then $\mathcal{T} \in \mathcal{D}(\lambda, n)$ if and only if $\mathcal{S} \in \mathcal{K N}(\lambda, n)$.

Proof. Let $\mathcal{P}$ and $\mathcal{Q}$ be admissible columns with entries from [[n]] and associated subsets $A_{\mathcal{P}}, B_{\mathcal{P}}, C_{\mathcal{P}}, D_{\mathcal{P}}$ and $A_{\mathcal{Q}}, B_{\mathcal{Q}}, C_{\mathcal{Q}}, D_{\mathcal{Q}}$ respectively. Set $\mathcal{P}^{\prime}=F\left(B_{\mathcal{P}}, C_{\mathcal{P}}\right)$ and $\mathcal{Q}^{\prime}=F\left(B_{\mathcal{Q}}, C_{\mathcal{Q}}\right)$. By comparing definitions of $\mathcal{K} \mathcal{N}(\lambda, n)$ and $\mathcal{D}(\lambda, n)$, the proof of the theorem easily reduces to showing that $\mathcal{P} \leq_{\mathcal{D}} \mathcal{Q}$ if and only if $\mathcal{P}^{\prime} \leq_{\mathcal{K N}} \mathcal{Q}^{\prime}$.

Assume $\mathcal{P} \leq_{\mathcal{D}} \mathcal{Q}$. Let $A, B, C, D$ be the associated subsets of an arbitrary admissible column. Since $F(A, C) \leq F(B, C) \leq F(B, D)$ and $A \cap C=B \cap D=\varnothing$, it is easily seen that $F(A, C) \leq_{\mathcal{K N}} F(B, C) \leq_{\mathcal{K N}} F(B, D)$ : the condition (II) is vacuously satisfied. By assumption we have $r \mathcal{P}=F\left(B_{\mathcal{P}}, D_{\mathcal{P}}\right) \leq F\left(A_{\mathcal{Q}}, C_{\mathcal{Q}}\right)=l \mathcal{Q}$. Since condition (II) for these two columns is vacuously satisfied we have $r \mathcal{P} \leq_{\mathcal{K N}}$ $l \mathcal{Q}$. The result $\mathcal{P}^{\prime} \leq_{\mathcal{K N} N} \mathcal{Q}^{\prime}$ now follows from transitivity: $\mathcal{P}^{\prime} \leq_{\mathcal{K N}} r \mathcal{P} \leq_{\mathcal{K N}} l \mathcal{Q} \leq_{\mathcal{K N}}$ $\mathcal{Q}^{\prime}$.

Now assume $\mathcal{P}^{\prime} \leq_{\mathcal{K N}} \mathcal{Q}^{\prime}$. We must show $\mathcal{P} \leq_{\mathcal{D}} \mathcal{Q}$. First we show $r \mathcal{P} \leq \mathcal{Q}^{\prime}$. Let $\left\{\mathbf{x}_{i}\right\}=\mathcal{P}^{\prime},\left\{\mathbf{y}_{i}\right\}=r \mathcal{P}$, and $\left\{\mathbf{z}_{i}\right\}=\mathcal{Q}^{\prime}$. Suppose that $r \mathcal{P} \not \leq \mathcal{Q}^{\prime}$. Note that the barred entries of $\mathcal{P}^{\prime}$ and $r \mathcal{P}$ are identical. Note also that $\mathcal{P}^{\prime} \leq_{\mathcal{K N}} \mathcal{Q}^{\prime}$ implies $\mathcal{P}^{\prime} \leq \mathcal{Q}^{\prime}$. Thus $r \mathcal{P} \not \leq \mathcal{Q}^{\prime}$ implies that there must be an $s$ such that $\mathbf{x}_{s}, \mathbf{y}_{s}, \mathbf{z}_{s}$ are all unbarred entries and $\mathbf{x}_{s} \leq \mathbf{z}_{s}<\mathbf{y}_{s}$. Let $\left\{d_{1}<d_{2}<\cdots<d_{k}\right\}$ list the unused slots $[n]-\left(A_{\mathcal{P}} \cup B_{\mathcal{P}} \cup C_{\mathcal{P}} \cup D_{\mathcal{P}}\right)$ of $\mathcal{P}$ and set $d_{0}=0$ and $d_{k+1}=n+1$. Lemma 8.2(2) implies there exists an $i, 0 \leq i \leq k$, such that $d_{i}<\left\|\mathbf{x}_{s}\right\|<\left\|\mathbf{y}_{s}\right\|<d_{i+1}$. Let 
$b$ (respectively $b^{\prime}$ ) be the slot of the leftmost top (respectively bottom) row circle in $\mathcal{P}^{\prime}$ to the right of $d_{i}$. Then $b^{\prime} \leq\left\|\mathbf{x}_{s}\right\| \leq\left\|\mathbf{z}_{s}\right\|$. Let $a$ be the slot of the rightmost circle in the top row of $\mathcal{P}^{\prime}$ strictly to the left of $\left\|\mathbf{y}_{s}\right\|$ : Since $\mathbf{x}_{s}<\mathbf{y}_{s}$ implies there is a full slot in $\mathcal{P}^{\prime}$ weakly between $d_{i}+1$ and $\left\|\mathbf{x}_{s}\right\|$, such an $a$ exists with $b \leq a$. Let $\mathbf{x}_{p}=\overline{\mathbf{a}}, \mathbf{x}_{q}=\overline{\mathbf{b}}$ and $\mathbf{x}_{r}=\mathbf{b}^{\prime}$. Then by Lemma A.2 we have

$$
(q-p)+(s-r)<\max \left(a\|\mathbf{z}\|_{s}\right)-\min \left(b, b^{\prime}\right) .
$$

We show that our assumption $\mathbf{z}_{s}<\mathbf{y}_{s}$ leads to a contradiction of this inequality. First we consider the right hand side of the inequality: Note that the circle diagram $\mathcal{P}_{\left(d_{i}, d_{i+1}\right)}$ consisting of the slots $d_{i}+1, \ldots, d_{i+1}-1$ of $\mathcal{P}$ is coadmissible. Since all of these slots are used we have $\min \left(b, b^{\prime}\right)=d_{i}+1$. Also note that $\max \left(a,\left\|\mathbf{z}_{s}\right\|\right)<\left\|\mathbf{y}_{s}\right\|$. Thus $\max \left(a,\left\|\mathbf{z}_{s}\right\|\right)-\min \left(b, b^{\prime}\right)<\left\|\mathbf{y}_{s}\right\|-\left(d_{i}+1\right)$. Now we interpret the left-hand side: consider the circle diagram $r \mathcal{P}_{\left(d_{i}\left\|\mathbf{y}_{s}\right\|\right]}$ consisting of the slots $d_{i}+1, \ldots,\left\|\mathbf{y}_{s}\right\|$ of $r \mathcal{P}$. Since the top row of the circle diagrams for $r \mathcal{P}$ and $\mathcal{P}^{\prime}$ are the same, there are $q-p+1$ circles in the top row of the circle diagram $r \mathcal{P}_{\left(d_{i},\left\|\mathbf{y}_{s}\right\|\right]}$. Since $\mathbf{x}_{r}=\mathbf{b}^{\prime}$ corresponds to the leftmost bottom row circle of $\mathcal{P}^{\prime}$ to the right of $d_{i}$, Lemma 8.2(2) implies that $\mathbf{y}_{r}$ corresponds to the leftmost bottom row circle of $r \mathcal{P}_{\left(d_{i},\left\|\mathbf{y}_{s}\right\|\right)}$. Thus there are $s-r+1$ circles in the bottom row of the circle diagram $r \mathcal{P}_{\left(d,\left\|\mathbf{y}_{s}\right\|\right]}$. All the slots of $r \mathcal{P}_{\left(d,\left\|\mathbf{y}_{s}\right\|\right]}$ are half-full since all the slots are used and there are no full slots. Thus there are as many circles as slots: $(q-p+1)+(s-r+1)=\left\|\mathbf{y}_{s}\right\|-d_{i}$. This implies $(q-p)+(s-r) \geq \max \left(a,\left\|\mathbf{z}_{s}\right\|\right)-\min \left(b, b^{\prime}\right)$ and we have arrived at our contradiction. Thus $\mathcal{P}^{\prime} \leq \mathcal{K N}_{\mathcal{N}} \mathcal{Q}^{\prime}$ implies $r \mathcal{P} \leq \mathcal{Q}^{\prime}$.

A similar argument shows that $\mathcal{P}^{\prime} \leq \mathcal{K N N}_{\mathcal{N}} \mathcal{Q}^{\prime}$ implies $\mathcal{P}^{\prime} \leq l \mathcal{Q}$. Consider the barred and unbarred entries of $r \mathcal{P}$ and $l \mathcal{Q}$ separately to see that these two results yield $r \mathcal{P} \leq l \mathcal{Q}$. Hence $\mathcal{P} \leq_{\mathcal{D}} \mathcal{Q}$.

\section{REFERENCES}

[And] G. E. Andrews, Plane partitions V: the T.S.S.C.P.P. conjecture, J. Comb. Theory Ser. A 66 (1994), 28-39. MR 95g:05010

[Ber] Allan Berele, A Schensted-type correspondence for the symplectic group, J. Comb. Theory Series A 43 (1986), 320-328. MR 88b:20027

[BZ] A. D. Berenstein and A. V. Zelevinsky, Tensor product multiplicities and convex polytopes in partition space, J.G.P. 5 (1988), 453-472. MR 91k:17003

[DeC] C. De Concini, Symplectic standard tableaux, Advances in Math. 34 (1979), 1-27. MR 80m:14036

[DP] C. DeConcini and C. Procesi, A characteristic free approach to invariant theory, Advances in Math. 21 (1976), 330-354. MR 54:10305

[DKR] J. Désarménien, J. Kung, and G.-C. Rota, Invariant theory, Young bitableaux, and combinatorics, Advances in Math. 27 (1978), 63-92. MR 58:5737

[E] K. Eriksson, Strongly convergent games and Coxeter groups, Dissertation, Kungl Tekniska Hogskolan, Stockholm, Sweden (May 1993).

[Kas] M. Kashiwara, On crystal bases of q-analogue of universal enveloping algebras, Duke Math. J. 63 (1991), 456-516. MR 93b:17045

[KN] M. Kashiwara and T. Nakashima, Crystal graphs for representations of the q-analogue of classical Lie algebras, J. Algebra 165 (1994), 295-345. MR 95c:17025

[Kng] R. C. King, Weight multiplicities for the classical groups, Lecture Notes in Physics 50 (New York; Springer 1975), 490-499. MR 58:1044

[Knu] D. E. Knuth, Permutation matrices and generalized Young tableaux, Pacific J. Math. 34 (1970), 709-727. MR 42:7535

[Kra] C. Krattenthaler, A bijection between Proctor's and Sundaram's odd orthogonal tableaux, Discrete Math. 161 (1996), 101-120. MR 91a:05152

[LMS] V. Lakshmibai, C. Musili, and C. S. Seshadri, Geometry of G/P, Bull. of AMS 1 (1979), 432-435. MR 81f: 14030 
[LLT] A. Lascoux, B. Leclerc, and J. Thibon, Crystal graphs and q-analogues of weight multiplicities for the root system $A_{n}$, Lett. Math. Phys. 35 (1995), 359-374. MR 97a:05213

[Lit1] Peter Littelmann, A generalization of the Littlewood-Richardson rule, J. Algebra 130 (1990), 328-368. MR 91f:22023

[Lit2] Peter Littelmann, A Littlewood-Richardson rule for symmetrizable Kac-Moody algebras, Invent. Math. 116 (1994), 329-346. MR 95f:17023

[Lit3] Peter Littelmann, A plactic algebra for semisimple Lie algebras, Advances in Math. 124 (1996), 312-331. MR 98c:17009

[Pro1] R. Proctor, New symmetric plane partition identities from invariant theory work of De Concini and Procesi, Europ. J. Combin. 11 (1990), 289-300. MR 91h:05010

[Pro2] R. Proctor, Four proofs of the Littlewood-Richardson rule, unpublished manuscript, 1986.

[Rob] D. Robbins, The story of 1,2, 7, 42,429,7436, .., Math. Intelligencer 13 (1991), 12-19. MR 92d:05010

[Sta] Richard P. Stanley, Symmetries of plane partitions, J. Comb. Thry Ser. A 43 (1986), 103-113. MR 87m:05017a

[Stro] Jeffrey Stroomer, Combinatorics and the representation the of $\operatorname{gl}(r, C)$ and $\operatorname{sp}(2 r, C)$, Dissertation, Univ. Wisconsin-Madison (1991).

[Sun] Sheila Sundaram, On the combinatorics of representations of $\operatorname{sp}(2 n, C)$, Dissertation, M.I.T. (1986).

[Zeil] Doron Zeilberger, Proof of the alternating sign matrix conjecture, The Foata Festschrift. Electron. J. Combin. 3 (1996) Research Paper 13. MR 97d:05012

[Zhl] D. P. Zhelobenko, Compact Lie Groups and Their Representations, Translations of Mathematical Monographs, vol. 40, American Mathematical Society, Providence, RI, 1973. MR $57: 12776 b$

Department of Mathematics, University of North Carolina, Chapel Hill, North CarOLINA 27599

Current address: 7364 E10th Avenue, Denver, Colorado 80220 